\title{
Contact geometric descriptions of vector fields on dually flat spaces and their applications in electric circuit models and nonequilibrium statistical mechanics
}

\author{
Shin-itiro Goto* \\ Institute for Molecular Science, \\ 38 Nishigo-Naka, Myodaiji, Okazaki 444-8585, Japan
}

July 10, 2018

\begin{abstract}
Contact geometry has been applied to various mathematical sciences, and it has been proposed that a contact manifold and a strictly convex function induce a dually flat space that is used in information geometry. Here, such a dually flat space is related to a Legendre submanifold in a contact manifold. In this paper contact geometric descriptions of vector fields on dually flat spaces are proposed on the basis of the theory of contact Hamiltonian vector fields. Based on these descriptions, two ways of lifting vector fields on Legendre submanifolds to contact manifolds are given. For some classes of these lifted vector fields, invariant measures in contact manifolds and stability analysis around Legendre submanifolds are explicitly given. Throughout this paper, Legendre duality is explicitly stated. In addition, to show how to apply these general methodologies to applied mathematical disciplines, electric circuit models and some examples taken from nonequilibrium statistical mechanics are analyzed.
\end{abstract}

\section{Introduction}

Contact geometry is often referred to as an odd-dimensional counterpart of symplectic geometry and then it has been studied from purely mathematical viewpoints 1. Aside from its purely mathematical interest, there are several applications in science and foundation of engineering. These applications include equilibrium thermodynamics 2, 3] 4], nonequilibrium thermodynamics [5] [, statistical mechanics [7 8] 9], fluid mechanics [10, electromagnetism[11, control theory [12, statistical theory for non-conservative system [13] and so on. In general, if geometric theories of mathematical disciplines are ascribed to a same geometry, then it can be expected that there are links among these disciplines. These links may give a unified picture of such disciplines. Such an example is found in contact geometry, where information geometry is linked to contact geometric thermodynamics [6] 7].

There are at least three key objects in contact geometry for the applications mentioned above. The first one is a class of vector fields, the second one a class of submanifolds, and the third one the total Legendre transform. The first one is canonical vector field called contact Hamiltonian vector field. Such vector fields are in some sense analogous to Hamiltonian vector fields in symplectic geometry. It has been known that such a vector field is specified by a function defined on a contact manifold. This function is called a contact Hamiltonian. With this class of vector fields, one is able to describe time dependent phenomena by identifying a parameter of an integral curve with time. The second one is a class of Legendre submanifolds. Here, Legendre submanifold is defined as a maximal dimensional integrable submanifold of a contact manifold. Such a submanifold can locally be specified with a function on a contact manifold, called a generating function of a Legendre submanifold. Thus, integrable submanifolds in contact manifolds can locally be described by generating functions. The third one is the total Legendre transform, and is well-known in mathematical sciences and foundation of engineering. This transform

\footnotetext{
*Present affiliation : Department of Applied Mathematics and Physics, Graduate School of Informatics, Kyoto University, Yoshida-Honmachi, Sakyo-ku, Kyoto 606-8501, Japan
} 
acting on functions enables one to change coordinates systematically, and gives different views such as Hamiltonian and Lagrangian formulations in classical mechanics. When there is a pair of mathematical statements ascribed to the total Legendre transform, such a pair is referred to as Legendre duality. In geometric thermodynamics formulated with contact geometry, these three keys are essential. Contact Hamiltonian vector fields are used for describing thermodynamic processes, Legendre submanifolds are used for specifying a subspace where the first law of thermodynamics is satisfied, and the total Legendre transform is used as well as non-geometric thermodynamics.

An approach to develop such contact geometric theories of mathematical sciences is therefore to give various relations on these three keys. One subject expected to be developed with the three keys in contact geometry is information geometry, where information geometry is a geometrization of mathematical statistics 14. As briefly mentioned earlier in this section, it has been pointed out that information geometry is connected to contact geometry. Although ideas in information geometry and those in contact geometry are well-developed, they have not been communicated. We then feel that a way to connect between them should be explored. Another approach to develop contact geometric theories is to give lifted vector fields on contact manifolds from those on Legendre submanifolds. This is because in contact geometric thermodynamics and in information geometry, vector fields are often studied only on manifolds that are effectively Legendre submanifolds of contact manifolds, and some extension of vector fields, not only on Legendre submanifolds, is expected to new applications. This class of lifts has been discussed in the literature. In Ref. [15], an equivalence of vector fields lifted to a contact manifold and stability of fixed points of contact vector fields being restricted to the Legendre submanifold has been discussed. In Ref. [16], it has been proposed how to solve the problem of matching two vector fields in thermodynamic systems, and stability of a class of dynamical systems lifted from a Legendre submanifold has been analyzed without any Lyapunov function on a contact manifold.

In this paper, some basic notations are fixed in 92 . In 93 it is shown that vector fields on dually flat spaces can be described as restricted contact Hamiltonian vector fields on Legendre submanifolds, where such restricted vector fields have been developed in the context of contact geometric thermodynamics. In this way, some theorems in information geometry are rewritten in terms of a contact geometric language. By showing that electric circuit models can be seen as dynamical systems on dually flat spaces, we argue how the present contact geometric and information geometric methodologies apply to engineering problems. In 4 it is shown that a class of flows defined on Legendre submanifolds is lifted to a contact manifold, and shown that lifted flows of some classes asymptotically approach to the Legendre submanifolds. Statistical properties such as phase compressibilities and invariant measures for lifted vector fields are argued as well. To show how these general theories can be used in physics, examples are constructed in nonequilibrium statistical mechanics. Since a relaxation dynamics of a spin model in contact with time-independent heat bath and time-independent external magnetic field has been well-described with contact geometry in Ref. [6], an extension of this dynamics may be of interest. A modification of such system is considered in this article, and it is shown that a relaxation process can be described in a system that is in contact with a time-dependent external magnetic field and timeindependent heat bath. As another example, a class of phenomenological equations with the Onsager coefficients is also discussed. In \$5 another scheme of lifting vector fields is shown where the values of generating functions for Legendre submanifolds are conservedalong vector fields. In this scheme, higher dimensional contact manifolds are to be employed. Statistical properties of such lifted vector fields are discussed as well. To show how this scheme is applied to engineering problems,electric circuit models in a thermal environment are analyzed. Throughout this paper, Legendre duality for mathematical statements is explicitly stated.

\section{Preliminaries}

In this section we give a brief summary of contact geometry and information geometry in order to describe statements that will be shown in Secs 35 .

\subsection{Mathematical symbols, definitions, and known facts}

Throughout this paper, geometric objects are assumed smooth. A point on an $n$-dimensional manifold $\xi \in \mathcal{M}$ is often identified with a set of values of local coordinates $x(\xi)=\left\{x^{1}(\xi), \ldots, x^{n}(\xi)\right\}$. A set of 
vector fields on a manifold $\mathcal{M}$ is denoted $\Gamma T \mathcal{M}$, the tangent space at $\xi \in \mathcal{M}$ as $T_{\xi} \mathcal{M}$, the cotangent space at $\xi \in \mathcal{M}$ as $T_{\xi}^{*} \mathcal{M}$, a set of $q$-form fields $\Gamma \Lambda^{q} \mathcal{M}$ with $q \in\{0, \ldots, \operatorname{dim} \mathcal{M}\}$, and a set of tensor fields $\Gamma T_{q}^{q^{\prime}} \mathcal{M}$ with $q, q^{\prime} \in\{0,1, \ldots\}$. If a tensor field $g \in \Gamma T_{2}^{0} \mathcal{M}$ on an $n$-dimensional manifold $\mathcal{M}$ satisfies (i) $\left.g\right|_{\xi}(X, Y)=\left.g\right|_{\xi}(Y, X)$, (ii) $\left.g\right|_{\xi}(X, X) \geq 0$, (iii) $\left.g\right|_{\xi}(X, X)=0$ iff $X=0$, for $X, Y \in T_{\xi} \mathcal{M}$, then $g$ is referred to as a Riemannian metric tensor field. An $n$-dimensional manifold together with a Riemannian metric tensor field $g$ is denoted $(\mathcal{M}, g)$ and this is referred to as an $n$-dimensional Riemannian manifold. If a tensor field $g \in \Gamma T_{2}^{0} \mathcal{M}$ on an $n$-dimensional manifold $\mathcal{M}$ satisfies (i) $\left.g\right|_{\xi}(X, Y)=\left.g\right|_{\xi}(Y, X)$, (ii) $\left.g\right|_{\xi}(Z, Y)=0,\left(\forall Z \in T_{\xi} \mathcal{M}\right) \Longrightarrow Y=0$, for $X, Y \in T_{\xi} \mathcal{M}$, then $g$ is referred to as a pseudo-Riemannian metric tensor field. An $n$-dimensional manifold together with a pseudo-Riemannian metric tensor field $g$ is denoted $(\mathcal{M}, g)$ and this is referred to as an $n$-dimensional pseudo-Riemannian manifold. To express tensor fields the direct product is denoted $\otimes$. Einstein notation, when an index variables appear twice in a single term it implies summation of all the values of the index, is adopted. The Kronecker delta is denoted $\delta_{a}^{b}, \delta_{a b}, \ldots$, and its value is unity when $a=b$, and zero when $a \neq b$. The exterior derivative acting on $\Gamma \Lambda^{q} \mathcal{M}$ is denoted $\mathrm{d}: \Gamma \Lambda^{q} \mathcal{M} \rightarrow \Gamma \Lambda^{q+1} \mathcal{M}$, and the interior product operator with $X \in \Gamma T \mathcal{M}$ as $\imath_{X}: \Gamma \Lambda^{q} \mathcal{M} \rightarrow \Gamma \Lambda^{q-1} \mathcal{M}$. Given a map $\Phi$ between two manifolds, the pull-back is denoted $\Phi^{*}$, and the push-forward $\Phi_{*}$. Then one can define the Lie derivative acting on tensor fields with respect to $X \in \Gamma T \mathcal{M}$ denoted $\mathcal{L}_{X}: \Gamma T_{q}^{q^{\prime}} \mathcal{M} \rightarrow \Gamma T_{q}^{q^{\prime}} \mathcal{M}$. It follows that $\mathcal{L}_{X} \beta=\left(\imath_{X} \mathrm{~d}+\mathrm{d} \imath_{X}\right) \beta$, for any $\beta \in \Gamma \Lambda^{q} \mathcal{M}$, which is referred to as the Cartan formula. One can also define a derivative along a given vector field $X$, called the covariant derivative, denoted $\nabla_{X}: \Gamma T_{q}^{0} \mathcal{M} \rightarrow \Gamma T_{q}^{0} \mathcal{M}$. The action is explicitly given by specifying the connection coefficients $\Gamma_{a b}{ }^{c},(a, b, c \in\{1, \ldots, \operatorname{dim} \mathcal{M}\})$ such that $\nabla_{X_{a}} X_{b}=\Gamma_{a b}{ }^{c} X_{c}$ where $\left\{X_{1}, \ldots, X_{n}\right\} \in \Gamma T \mathcal{M}$ is a basis. For a given $S \in \Gamma T_{q}^{0} \mathcal{M}$, an object $\nabla S \in \Gamma T_{q+1}^{0} \mathcal{M}$ is defined such that $(\nabla S)\left(X, Y_{1}, \ldots, Y_{q}\right)=\left(\nabla_{X} S\right)\left(Y_{1}, \ldots, Y_{q}\right)$, where $X, Y_{1}, \ldots, Y_{q} \in \Gamma T \mathcal{M}$. For example it can be shown that $\nabla f=\mathrm{d} f$ for $f \in \Gamma T_{0}^{0} \mathcal{M}$, and that $(\nabla \mathrm{d} f)\left(Y, \partial / \partial x^{a}\right)=Y^{b}\left(\partial^{2} f / \partial x^{a} \partial x^{b}-\Gamma_{b a}{ }^{c} \partial f / \partial x^{c}\right)$, where $Y=Y^{b} \partial / \partial x^{b} \in \Gamma T \mathcal{M}$.

Definition 2.1. (Dynamical system and its flow) : Let $\mathcal{M}$ be an $n$-dimensional manifold, $x=$ $\left\{x^{1}, \ldots, x^{n}\right\}$ a local coordinate system, $\mathbb{T}$ a subset of the real numbers $\mathbb{R}$, and $F=\left\{F^{1}, \ldots, F^{n}\right\}$ a set of functions on $\mathcal{M}$. Consider a set of ordinary differential equations

$$
\frac{\mathrm{d} x^{a}}{\mathrm{~d} t}=F^{a}(x) . \quad t \in \mathbb{T} \subset \mathbb{R}
$$

If there exists the unique solution $\phi\left(t, x_{0}\right)$ with $x_{0}$ being the initial point at $t=0$ for all $x_{0} \in \mathcal{M}$ and all $t \in \mathbb{T}$, then this set of ordinary differential equations is referred to as a dynamical system on $\mathcal{M}$. Define $\phi_{t}: \mathcal{M} \rightarrow \mathcal{M}$ with a fixed $t \in \mathbb{T}$ such that $\phi_{t}=\phi(t,-)$. Then, $\left\{\phi_{t}\right\}_{t \in \mathbb{T}}$ is referred to as a flow. In addition, one can have a vector field $X=F^{a} \partial / \partial x^{a}$ on $\mathcal{M}$. This $X$ is referred to as a vector field associated with a dynamical system.

Definition 2.2. ( Volume-form divergence and phase compressibility, [17], [18] ) : Let $\mathcal{M}$ be an $n$ dimensional manifold, $X$ a vector field on $\mathcal{M}$, and $\Omega$ a non-vanishing $n$-form. Define $\kappa_{\Omega}: T_{\xi} \mathcal{M} \rightarrow \mathbb{R}$, $(\xi \in \mathcal{M})$ such that

$$
\mathcal{L}_{X} \Omega=\kappa_{\Omega}(X) \Omega \text {. }
$$

Then, $\kappa_{\Omega}(X)$ is referred to as a volume-form divergence. If $X$ is a vector filed associated with a dynamical system on $\mathcal{M}$, then $\kappa_{\Omega}(X)$ is referred to as a phase compressibility.

Remark 2.1. In the context of dynamical systems theory, volume-form divergences are simply referred to as divergences, where a volume-form is defined in differential geometry as an $n$-form that does not vanish on any point on $\mathcal{M}$. In this paper, another divergence in a different context will be introduced and that will be distinguished from the volume-form divergence.

Definition 2.3. ( Continuity equation for volume form and invariant measure, [17], 18] ) : Let $\mathcal{M}$ be an $n$-dimensional manifold, $\mathbb{T}$ a subset of $\mathbb{R}, \Omega$ a non-vanishing $n$-form that can depend on $t \in \mathbb{T}$, and $X$ a vector field associated with a dynamical system $\mathrm{d} x^{a} / \mathrm{d} t=F^{a}(x),(a \in\{1, \ldots, n\})$ with $\left\{F^{1}, \ldots, F^{n}\right\}$ being functions. The equation for $\Omega$

$$
\frac{\partial}{\partial t} \Omega+\mathcal{L}_{X} \Omega=0
$$

is referred to as the continuity equation for $\Omega$. In addition, a solution that satisfies

$$
\frac{\partial}{\partial t} \Omega=0, \quad \text { and } \quad \mathcal{L}_{X} \Omega=0
$$


is referred to as an invariant measure for $X$.

Remark 2.2. The equation for a function $f$

$$
\frac{\partial f}{\partial t}+(X f)+\kappa_{\Omega}(X) f=0
$$

is referred to as the generalized Liouville equation. This is often studied in the literature, and is derived from the continuity equation for $\Omega$ as follows. Substituting $\Omega=f \Omega_{0}$ with $\Omega_{0}$ being a non-vanishing $n$-form that does not depend on $t$ into the continuity equation for $\Omega$, one has that

$$
\left[\frac{\partial}{\partial t} f+(X f)\right] \Omega_{0}+f \mathcal{L}_{X} \Omega_{0}=0 .
$$

With this, $\mathcal{L}_{X} \Omega_{0}=\kappa_{\Omega_{0}}(X) \Omega_{0}$ as in (1), and $\Omega_{0}$ is then rewritten as $\Omega$, one has the generalized Liouville equation.

Definition 2.4. ( Contact manifold) : Let $\mathcal{C}$ be a $(2 n+1)$-dimensional manifold, and $\lambda$ a one-form on $\mathcal{C}$ such that

$$
\lambda \wedge \underbrace{\mathrm{d} \lambda \wedge \mathrm{d} \lambda \cdots \wedge \mathrm{d} \lambda}_{n} \neq 0
$$

at any point on $\mathcal{C}$. If $\mathcal{C}$ carries $\lambda$, then $(\mathcal{C}, \lambda)$ is referred to as a contact manifold and $\lambda$ a contact form.

It should be noted that there are other definitions of contact manifold. However the definition above is used in this paper. The following definition is not commonly used in the literature.

Definition 2.5. ( Standard volume-form) : The $(2 n+1)$-form in Definition 2.4 is referred to as the standard volume-form :

$$
\Omega_{\lambda}=\lambda \wedge \mathrm{d} \lambda \wedge \cdots \wedge \mathrm{d} \lambda
$$

There is a standard local coordinate system.

Theorem 2.1. ( Existence of particular coordinates ) : There exist local $(2 n+1)$ coordinates $(x, p, z)$ with $x=\left\{x^{1}, \ldots, x^{n}\right\}$ and $p=\left\{p_{1}, \ldots, p_{n}\right\}$, in which $\lambda$ has the form

$$
\lambda=\mathrm{d} z-p_{a} \mathrm{~d} x^{a} .
$$

Definition 2.6. ( Canonical coordinates or Darboux coordinates ) : The $(2 n+1)$ coordinates $(x, p, z)$ introduced in Theorem 2.1 are referred to as the canonical coordinates, or the Darboux coordinates.

In addition to the above coordinates, ones in which $\lambda$ has the form $\lambda=\mathrm{d} z+p_{a} \mathrm{~d} x^{a}$ are also used in the literature. In this paper (3) is used.

Given a contact manifold, there exists a unique vector field that is defined as follows.

Definition 2.7. ( Reeb vector field or characteristic vector field) : Let $(\mathcal{C}, \lambda)$ be a contact manifold, and $\mathcal{R}$ a vector field on $\mathcal{C}$. If $\mathcal{R}$ satisfies

$$
\imath_{\mathcal{R}} \mathrm{d} \lambda=0, \quad \text { and } \quad \imath_{\mathcal{R}} \lambda=1,
$$

then $\mathcal{R} \in \Gamma T \mathcal{C}$ is referred to as the Reeb vector field, or the characteristic vector field.

When $\lambda$ is given, a coordinate expression for $\mathcal{R}$ is given as follows.

Proposition 2.1. ( Coordinate expression of the Reeb vector field) : Let $(\mathcal{C}, \lambda)$ be a contact manifold, and $\mathcal{R}$ the Reeb vector field. If the canonical coordinates $(x, p, z)$ are such that $\lambda=\mathrm{d} z \pm p_{a} \mathrm{~d} x^{a}$ with $x=\left\{x^{1}, \ldots, x^{n}\right\}$ and $p=\left\{p_{1}, \ldots, p_{n}\right\}$, then

$$
\mathcal{R}=\frac{\partial}{\partial z}
$$

The following submanifold plays various roles in applications of contact geometry. 
Definition 2.8. ( Legendre submanifold) : Let $(\mathcal{C}, \lambda)$ be a contact manifold, and $\mathcal{A}$ a submanifold of $\mathcal{C}$. If $\mathcal{A}$ is a maximal dimensional integral submanifold of $\lambda$, then $\mathcal{A}$ is referred to as a Legendre submanifold.

The following theorem states the dimension of a Legendre submanifold for a given contact manifold.

Theorem 2.2. ( Maximal dimensional integral submanifold) : On a $(2 n+1)$-dimensional contact manifold $(\mathcal{C}, \lambda)$, a maximal dimensional integral submanifold of $\lambda$ is equal to $n$.

Combining Theorem 2.2 and Definition 2.8, one concludes the following.

Theorem 2.3. (Dimension of Legendre submanifolds ) : The dimension of any Legendre submanifold of a $(2 n+1)$-dimensional contact manifold is $n$.

The following theorem shows the explicit local expressions of Legendre submanifolds in terms of canonical coordinates.

Theorem 2.4. ( Local expressions of Legendre submanifolds, [1]) : Let $(\mathcal{C}, \lambda)$ be a $(2 n+1)$-dimensional contact manifold, and $(x, p, z)$ the canonical coordinates such that $\lambda=\mathrm{d} z-p_{a} \mathrm{~d} x^{a}$ with $x=\left\{x^{1}, \ldots, x^{n}\right\}$ and $p=\left\{p_{1}, \ldots, p_{n}\right\}$. For any partition $I \cup J$ of the set of indices $\{1, \ldots, n\}$ into two disjoint subsets $I$ and $J$, and for a function $\phi\left(x^{J}, p_{I}\right)$ of $n$ variables $p_{i}, i \in I$, and $x^{j}, j \in J$ the $(n+1)$ equations

$$
x^{i}=-\frac{\partial \phi}{\partial p_{i}}, \quad p_{j}=\frac{\partial \phi}{\partial x^{j}}, \quad z=\phi-p_{i} \frac{\partial \phi}{\partial p_{i}}
$$

define a Legendre submanifold. Conversely, every Legendre submanifold of $(\mathcal{C}, \lambda)$ in a neighborhood of any point is defined by these equations for at least one of the $2^{n}$ possible choices of the subset $I$.

Definition 2.9. ( Legendre submanifold generated by a function ) : The function $\phi$ used in Theorem 2.4 is referred to as a generating function of the Legendre submanifold. If a Legendre submanifold $\mathcal{A}$ is expressed as (6), then $\mathcal{A}$ is referred to as a Legendre submanifold generated by $\phi$.

The following are examples of local expressions for Legendre submanifolds.

Example 2.1. Let $(\mathcal{C}, \lambda)$ be a $(2 n+1)$-dimensional contact manifold, $(x, p, z)$ the canonical coordinates such that $\lambda=\mathrm{d} z-p_{a} \mathrm{~d} x^{a}$ with $x=\left\{x^{1}, \ldots, x^{n}\right\}$ and $p=\left\{p_{1}, \ldots, p_{n}\right\}$, and $\psi$ a function of $x$ only. The Legendre submanifold $\mathcal{A}_{\psi}$ generated by $\psi$ with $\Phi_{\mathcal{C} \mathcal{A} \psi}: \mathcal{A}_{\psi} \rightarrow \mathcal{C}$ being an embedding is such that

$$
\Phi_{\mathcal{C} \mathcal{A} \psi} \mathcal{A}_{\psi}=\left\{(x, p, z) \in \mathcal{C} \mid p_{j}=\frac{\partial \psi}{\partial x^{j}}, \text { and } z=\psi(x), \quad j \in\{1, \ldots, n\}\right\} .
$$

The relation between this $\psi$ and $\phi$ of (6) is $\psi(x)=\phi(x)$ with $J=\{1, \ldots, n\}$. One can verify that $\Phi_{\mathcal{C} \mathcal{A} \psi}{ }^{*} \lambda=0$.

Example 2.2. Let $(\mathcal{C}, \lambda)$ be a $(2 n+1)$-dimensional contact manifold, $(x, p, z)$ the canonical coordinates such that $\lambda=\mathrm{d} z-p_{a} \mathrm{~d} x^{a}$ with $x=\left\{x^{1}, \ldots, x^{n}\right\}$ and $p=\left\{p_{1}, \ldots, p_{n}\right\}$, and $\varphi$ a function of $p$ only. The Legendre submanifold $\mathcal{A}_{\varphi}$ generated by $-\varphi$ with $\Phi_{\mathcal{C A} \varphi}: \mathcal{A}_{\varphi} \rightarrow \mathcal{C}$ being an embedding is such that

$$
\Phi_{\mathcal{C A} \varphi} \mathcal{A}_{\varphi}=\left\{(x, p, z) \in \mathcal{C} \mid x^{i}=\frac{\partial \varphi}{\partial p_{i}}, \text { and } z=p_{i} \frac{\partial \varphi}{\partial p_{i}}-\varphi(p), \quad i \in\{1, \ldots, n\}\right\} .
$$

The relation between this $\varphi$ and $\phi$ of (66) is $\varphi(p)=-\phi(p)$ with $I=\{1, \ldots, n\}$. One can verify that $\Phi_{\mathcal{C A} \varphi}{ }^{*} \lambda=0$.

One can choose a function $\psi$ in Example2.1 to generate $\mathcal{A}_{\psi}$, and $\varphi$ in Example 2.2 to generate $\mathcal{A}_{\varphi}$ independently, and in this case there is no relation between $\mathcal{A}_{\psi}$ and $\mathcal{A}_{\varphi}$ in general. On the other hand, when $\psi$ is strictly convex, and $\varphi$ is carefully chosen, it can be shown that there is a relation between $\mathcal{A}_{\psi}$ and $\mathcal{A}_{\varphi}$. To discuss such a case, the following transform should be introduced. The convention is adopted to that in information geometry. Note that several conventions exist in the literature.

Definition 2.10. ( Total Legendre transform ) : Let $\mathcal{M}$ be an $n$-dimensional manifold, $x=\left\{x^{1}, \ldots, x^{n}\right\}$ coordinates, and $\psi$ a function of $x$. Then the total Legendre transform of $\psi$ with respect to $x$ is defined to be

$$
\mathfrak{L}[\psi](p):=\sup _{x}\left[x^{a} p_{a}-\psi(x)\right]
$$

where $p=\left\{p_{1}, \ldots, p_{n}\right\}$ 
From this definition, one has several formulae that will be used in Secs 35

Theorem 2.5. (Formulae involving the total Legendre transform) : Let $\mathcal{M}$ be an $n$-dimensional manifold, $x=\left\{x^{1}, \ldots, x^{n}\right\}$ coordinates, $\psi \in \Gamma \Lambda^{0} \mathcal{M}$ a strictly convex function of $x$ only, and $\varphi$ the function of $p$ obtained by the total Legendre transform of $\psi$ with respect to $x$ where $p=\left\{p_{1}, \ldots, p_{n}\right\}$ : $\varphi(p)=\mathfrak{L}[\psi](p)$. Then, for each a and fixed $p$, the equation

$$
p_{a}=\left.\frac{\partial \psi(x)}{\partial x^{a}}\right|_{x=x_{*}}=\frac{\partial \psi\left(x_{*}\right)}{\partial x_{*}^{a}},
$$

has the unique solution $x_{*}^{a}=x_{*}^{a}(p),(a \in\{1, \ldots, n\})$. In addition it follows that

$$
\varphi(p)=x_{*}^{a} p_{a}-\psi\left(x_{*}\right), \quad \frac{\partial \varphi}{\partial p_{a}}=x_{*}^{a}, \quad \delta_{b}^{a}=\frac{\partial^{2} \psi}{\partial x_{*}^{b} \partial x_{*}^{l}} \frac{\partial^{2} \varphi}{\partial p_{a} \partial p_{l}},
$$

and

$$
\operatorname{det}\left(\frac{\partial^{2} \psi}{\partial x^{a} \partial x^{b}}\right)>0, \quad \operatorname{det}\left(\frac{\partial^{2} \varphi}{\partial p_{a} \partial p_{b}}\right)>0 .
$$

A way to describe dynamics on a contact manifold is to introduce a continuous diffeomorphism with a parameter. First, one defines a diffeomorphism on a contact manifold.

Definition 2.11. ( Contact diffeomorphism) : Let $(\mathcal{C}, \lambda)$ be a $(2 n+1)$-dimensional contact manifold, and $\Phi: \mathcal{C} \rightarrow \mathcal{C}$ a diffeomorphism. If it follows that

$$
\Phi^{*} \lambda=f \lambda
$$

where $f \in \Gamma \Lambda^{0} \mathcal{C}$ is a function that does not vanish at any point of $\mathcal{C}$, then the map $\Phi$ is referred to as a contact diffeomorphism.

Remark 2.3. It follows that $\Phi$ preserves the contact structure, ker $\lambda:=\left\{X \in \Gamma T \mathcal{C} \mid \imath_{X} \lambda=0\right\}$, but does not preserve the original contact form.

In addition to this diffeomorphism, one can introduce one-parameter groups as follows.

Definition 2.12. (One-parameter group of continuous contact transformations ) : Let $(\mathcal{C}, \lambda)$ be a $(2 n+1)$-dimensional contact manifold, and $\Phi_{t}: \mathcal{C} \rightarrow \mathcal{C}$ a diffeomorphism with $t \in \mathbb{R}$ that satisfies $\Phi_{0}=\operatorname{Id}_{\mathcal{C}}$ and $\Phi_{t+s}=\Phi_{t} \circ \Phi_{s},(t, s \in \mathbb{R})$, where $\operatorname{Id}_{\mathcal{C}}$ is such that $\operatorname{Id} \mathcal{C} \xi \xi$ for all $\xi \in \mathcal{C}$. If it follows that

$$
\Phi_{t}^{*} \lambda=f_{t} \lambda
$$

where $f_{t} \in \Gamma \Lambda^{0} \mathcal{C}$ is a function that does not vanish at any point of $\mathcal{C}$, then the $\Phi_{t}$ is referred to as a one-parameter group of continuous contact transformations. If $t, s \in \mathbb{T}$ with some $\mathbb{T} \subset \mathbb{R}$, then it is referred to as a one-parameter local transformation group of continuous contact transformations.

A contact vector field is defined as follows.

Definition 2.13. ( Contact vector field) : Let $(\mathcal{C}, \lambda)$ be a contact manifold, and $X$ a vector field on $\mathcal{C}$. If $X$ satisfies

$$
\mathcal{L}_{X} \lambda=f \lambda,
$$

where $f$ is a functionon $\mathcal{C}$, then $X$ is referred to as a contact vector field.

A one-parameter (local) transformation groups is realized by integrating the following vector field.

Definition 2.14. (Contact vector field associated to a contact Hamiltonian) : Let ( $\mathcal{C}, \lambda$ ) be a contact manifold, $\mathcal{R}$ the Reeb vector field, $h$ a function on $\mathcal{C}$, and $X_{h}$ a vector field. If $X_{h} \in \Gamma T \mathcal{C}$ satisfies

$$
\imath_{X} \lambda=h, \quad \text { and } \quad \imath_{X_{h}} \mathrm{~d} \lambda=-(\mathrm{d} h-(\mathcal{R} h) \lambda),
$$

then $X_{h}$ is referred to as a contact vector field associated to a function $h$ or a contact Hamiltonian vector field. In addition $h$ is referred to as a contact Hamiltonian. 
Remark 2.4. The definition (10) and the Cartan formula give

$$
\mathcal{L}_{X_{h}} \lambda=(\mathcal{R} h) \lambda \text {. }
$$

Remark 2.5. With (10), (11), and the formula $\mathcal{L}_{X} \imath_{X} \alpha=\imath_{X} \mathcal{L}_{X} \alpha$, where $X$ is an arbitrary vector field and $\alpha$ an arbitrary $q$-form field $(q \in\{0,1, \ldots\})$, one has that

$$
X_{h} h=\mathcal{L}_{X_{h}} h=\mathcal{L}_{X_{h}}\left(\imath_{X_{h}} \lambda\right)=\imath_{X_{h}}\left(\mathcal{L}_{X_{h}} \lambda\right)=(\mathcal{R} h)\left(\imath_{X_{h}} \lambda\right)=(\mathcal{R} h) h .
$$

From (11) and Definition 2.13, one has the following.

Theorem 2.6. ( Relation between a contact Hamiltonian vector field and a contact vector field) : Let $(\mathcal{C}, \lambda)$ be a contact manifold, $h$ a contact Hamiltonian, and $X_{h}$ a contact Hamiltonian vector field. Then $X_{h}$ is a contact vector field.

Local expressions of a contact Hamiltonian vector field (10) are calculated as follows.

Proposition 2.2. ( Local expression of contact Hamiltonian vector field) : Let $(\mathcal{C}, \lambda)$ be a $(2 n+$ 1)-dimensional contact manifold, $h$ a contact Hamiltonian, $X_{h}$ a contact Hamiltonian vector field, and $(x, p, z)$ the canonical coordinates such that $\lambda=\mathrm{d} z-p_{a} \mathrm{~d} x^{a}$ with $x=\left\{x^{1}, \ldots, x^{n}\right\}$ and $p=$ $\left\{p_{1}, \ldots, p_{n}\right\}$. Then

$$
X_{h}=\dot{x}^{a} \frac{\partial}{\partial x^{a}}+\dot{p}_{a} \frac{\partial}{\partial p_{a}}+\dot{z} \frac{\partial}{\partial z},
$$

where' denotes the differential with respect to a parameter $t \in \mathbb{R}$, or $t \in \mathbb{T}$ with some $\mathbb{T} \subset \mathbb{R}$, and

$$
\dot{x}^{a}=-\frac{\partial h}{\partial p_{a}}, \quad \dot{p}_{a}=\frac{\partial h}{\partial x^{a}}+p_{a} \frac{\partial h}{\partial z}, \quad \dot{z}=h-p_{a} \frac{\partial h}{\partial p_{a}}, \quad a \in\{1, \ldots, n\} .
$$

Remark 2.6. When $\lambda=\mathrm{d} z+p_{a} \mathrm{~d} x^{a}$, signs in (13) are changed.

The following theorem is well-known, and has been used in the literature of geometric thermodynamics.

Theorem 2.7. ( Tangent vector field of a Legendre submanifold realized by a contact Hamiltonian vector field, [19] ) : Let $(\mathcal{C}, \lambda)$ be a contact manifold, $\mathcal{A}$ a Legendre submanifold, and $h$ a contact Hamiltonian. Then the contact Hamiltonian vector field is tangent to $\mathcal{A}$ if and only if $h$ vanishes on $\mathcal{A}$.

The following definitions have been used mainly in information geometry, and are used in this paper as well.

Definition 2.15. (Affine-coordinate and flat connection, 14] ) Let $\mathcal{M}$ be an n-dimensional manifold, $x=\left\{x^{1}, \ldots, x^{n}\right\}$ coordinates, $\nabla$ a connection, $\left\{\Gamma_{a b}^{c}\right\}$ connection coefficients such that $\nabla_{\partial_{a}} \partial_{b}=\Gamma_{a b}{ }^{c} \partial_{c}$, $\left(\partial_{a}:=\partial / \partial x^{a}\right)$. If $\left\{\Gamma_{a b}^{c}\right\} \equiv 0$ hold for all $\xi \in \mathcal{M}$, then xis referred to as a $\nabla$-affine coordinate system, or affine coordinates. If it is the case, then $\nabla$ is referred to as a flat connection.

Definition 2.16. (Dual connection, [14] ) : Let $(\mathcal{M}, g)$ be an n-dimensional Riemannian or pseudoRiemannian manifold, and $\nabla$ a connection. If a connection $\nabla^{*}$ satisfies

$$
Z[g(X, Y)]=g\left(\nabla_{Z} X, Y\right)+g\left(X, \nabla_{Z}^{*} Y\right), \quad \forall X, Y, Z \in \Gamma T \mathcal{M}
$$

then $\nabla$ and $\nabla^{*}$ are referred to as dual connections, also $\nabla^{*}$ is referred to as a dual connection of $\nabla$ with respect to $g$.

Lemma 2.1. (Existence of a unique dual connection, 14] ) : Given a metric tensor field and a connection, there exists a unique dual connection .

Proof. Let $g$ and $\nabla$ be the given metric tensor field and connection, respectively. Then in what follows the explicit form of the dual connection $\nabla^{\prime}$ is shown. Let $x=\left\{x^{1}, \ldots, x^{n}\right\}$ be local coordinates, $\left\{g_{a b}\right\}$ the components of the metric tensor field such that $g=g_{a b} \mathrm{~d} x^{a} \otimes \mathrm{d} x^{b},\left\{\Gamma_{a b}{ }^{c}\right\}$ connection coefficients for $\nabla$ such that $\nabla_{\partial_{a}} \partial_{b}=\Gamma_{a b}^{c} \partial_{c}$ with $\partial_{a}:=\partial / \partial x^{a}$, and $\left\{\Gamma_{a b}^{\prime c}\right\}$ connection coefficients for $\nabla^{\prime}$ such that $\nabla_{\partial_{a}}^{\prime} \partial_{b}=\Gamma_{a b}^{\prime c} \partial_{c}$. Then, defining $\Gamma_{a b c}:=g_{c k} \Gamma_{a b}^{k}$ and $\Gamma_{a b c}^{\prime}:=g_{c k} \Gamma_{a b}^{\prime k}$, one can determine $\left\{\Gamma_{a b c}^{\prime}\right\}$ uniquely with (14) as $\Gamma_{a b c}^{\prime}=\partial_{c} g_{a b}-\Gamma_{a b c}$. 
Definition 2.17. ( Dual coordinate, [14] ) : Let $(\mathcal{M}, g)$ be an n-dimensional Riemannian or pseudoRiemannian manifold, $x=\left\{x^{1}, \ldots, x^{n}\right\}$ a set of local coordinates, and $p=\left\{p_{1}, \ldots, p_{n}\right\}$ another set of local coordinates. If

$$
g\left(\frac{\partial}{\partial x^{a}}, \frac{\partial}{\partial p_{b}}\right)=\delta_{a}^{b}
$$

then $p$ is referred to as the dual coordinate system. If it is the case, then $x$ and $p$ are referred to as being mutually dual with respect to $\mathrm{g}$.

Combining Definitions 2.15, 2.16 and 2.17, one has the following.

Lemma 2.2. (Dual coordinate and affine coordinate) : Let $(\mathcal{M}, g)$ be an $n$-dimensional Riemannian or pseudo-Riemannian manifold, $\nabla$ a connection, $x=\left\{x^{1}, \ldots, x^{n}\right\}$ a set of $\nabla$-affine coordinates, and $p=\left\{p_{1}, \ldots, p_{n}\right\}$ another set of coordinates. If $x$ and $p$ are mutually dual with respect to $g$, then $p$ is a $\nabla^{*}$-affine coordinate system.

Proof. There exists the connection $\nabla^{*}$ being dual of $\nabla$ due to Lemma2.1 It follows from (15) that

$$
Z\left[g\left(\frac{\partial}{\partial x^{a}}, \frac{\partial}{\partial p_{b}}\right)\right]=0
$$

for $\forall Z \in \Gamma T \mathcal{M}$. Then, with this and (14), one has

$$
Z\left[g\left(\frac{\partial}{\partial x^{a}}, \frac{\partial}{\partial p_{b}}\right)\right]=g\left(\nabla_{Z} \frac{\partial}{\partial x^{a}}, \frac{\partial}{\partial p_{b}}\right)+g\left(\frac{\partial}{\partial x^{a}}, \nabla_{Z}^{*} \frac{\partial}{\partial p_{b}}\right)=0 .
$$

Since $x$ is a $\nabla$-affine coordinate system, $\nabla_{Z}\left(\partial / \partial x^{a}\right)=0,(a \in\{1, \ldots, n\})$, one can conclude that $\nabla_{Z}^{*}\left(\partial / \partial p_{b}\right)=0,(b \in\{1, \ldots, n\})$, from which $p$ is a $\nabla^{*}$-affine coordinate system.

Definition 2.18. ( Dually flat space, [14] ) : Let $(\mathcal{M}, g)$ be an $n$-dimensional Riemannian or pseudoRiemannian manifold, $\nabla$ and $\nabla^{*}$ dual connections. If there exist $\nabla$-affine coordinates and $\nabla^{*}$-affine ones, then $\left(\mathcal{M}, g, \nabla, \nabla^{*}\right)$ is referred to as a dually flat space.

From these definitions, one can show the following relation between pairings and inner products.

Proposition 2.3. ( Inner products and pairings on a dually flat space) : Let $\left(\mathcal{M}, g, \nabla, \nabla^{*}\right)$ be an $n$-dimensional dually flat space, $x=\left\{x^{1}, \ldots, x^{n}\right\}$ a set of $\nabla$-affine coordinates, $p=\left\{p_{1}, \ldots, p_{n}\right\}$ a set of $\nabla^{*}$-affine coordinates. If the inner products $T_{\xi} \mathcal{M} \times T_{\xi} \mathcal{M} \rightarrow \mathbb{R},(\xi \in \mathcal{M})$ between the bases $\left\{\partial / \partial x^{1}, \ldots, \partial / \partial x^{n}\right\}$ and $\left\{\partial / \partial p_{1}, \ldots, \partial / \partial p_{n}\right\}$ are given as

$$
\begin{array}{ll}
g\left(\frac{\partial}{\partial x^{a}}, \frac{\partial}{\partial x^{b}}\right)=g_{a b}, & g\left(\frac{\partial}{\partial x^{a}}, \frac{\partial}{\partial p_{b}}\right)=\delta_{a}^{b}, \\
g\left(\frac{\partial}{\partial p_{a}}, \frac{\partial}{\partial x^{b}}\right)=\delta_{b}^{a}, & g\left(\frac{\partial}{\partial p_{a}}, \frac{\partial}{\partial p_{b}}\right)=g^{a b},
\end{array}
$$

(i.e., $x$ and $p$ are mutually dual with respect to $g$ ), then one has the following pairings $T_{\xi}^{*} \mathcal{M} \times T_{\xi} \mathcal{M} \rightarrow$ $\mathbb{R},(\xi \in \mathcal{M})$

$$
\begin{aligned}
\mathrm{d} x^{a}\left(\frac{\partial}{\partial x^{b}}\right) & =\delta^{a b}, & \mathrm{~d} x^{a}\left(\frac{\partial}{\partial p_{b}}\right) & =g^{a b}, \\
\mathrm{~d} p_{a}\left(\frac{\partial}{\partial x^{b}}\right) & =g_{a b}, & \mathrm{~d} p_{a}\left(\frac{\partial}{\partial p_{b}}\right) & =\delta_{a b} .
\end{aligned}
$$

As shown in Ref.[6], a contact manifold and a strictly convex function induce a dually flat space. The proof below is slightly different to that in Ref. [6].

Theorem 2.8. ( Contact manifold and a function induce a dually flat space, [6] ) : Let (C, $\lambda$ ) be a $(2 n+1)$-dimensional contact manifold, $(x, p, z)$ the canonical coordinates such that $\lambda=\mathrm{d} z-p_{a} \mathrm{~d} x^{a}$ with $x=\left\{x^{1}, \ldots, x^{n}\right\}$ and $p=\left\{p_{1}, \ldots, p_{n}\right\}$, and $\psi$ a strictly convex function of $x$ only. If the Legendre submanifold generated by $\psi$ is simply connected, then $((\mathcal{C}, \lambda), \psi)$ induces an $n$-dimensional dually flat space $\left(\Phi_{\mathcal{C} \mathcal{A} \psi} \mathcal{A}_{\psi}, g, \nabla, \nabla^{*}\right)$ with $\Phi_{\mathcal{C A} \psi}: \mathcal{A}_{\psi} \rightarrow \mathcal{C}$ being an embedding. 
Proof. In this proof, from $((\mathcal{C}, \lambda), \psi)$ we explicitly specify $g, \nabla, \nabla^{*}, \nabla$-affine coordinates, and $\nabla^{*}$ affine coordinates on the embedded Legendre submanifold generated by $\psi$.

Let $\varphi$ be the total Legendre transform of $\psi$ with respect to $x$. With the general theory of Legendre transform and the given strictly convex function $\psi$, one can define $x_{a}^{*}$ and have the relation such that

$$
x_{a}^{*}:=\frac{\partial \psi}{\partial x^{a}}, \quad x^{a}=\frac{\partial \varphi}{\partial x_{a}^{*}}, \quad \varphi\left(x^{*}\right)=\mathfrak{L}[\psi]\left(x^{*}\right) .
$$

On the embedded Legendre submanifold $\mathcal{A}_{\psi}^{\mathcal{C}}:=\Phi_{\mathcal{C A} \psi} \mathcal{A}_{\psi}^{\mathcal{C}}$, one has that $x_{a}^{*}=p_{a}$, due to (7). In what follows $g, \nabla, \nabla^{*}, \nabla$-affine coordinates, and $\nabla^{*}$-affine coordinates are explicitly specified on $\mathcal{A}_{\psi}^{\mathcal{C}}$. First, one defines

$$
g_{a b}(x):=\frac{\partial^{2} \psi}{\partial x^{a} \partial x^{b}}, \quad \text { and } \quad g^{a b}(p):=\frac{\partial^{2} \varphi}{\partial p_{a} \partial p_{b}} .
$$

It can be shown that $g_{a j} g^{j b}=\delta_{a}^{b}$. It follows from $\operatorname{det}\left(g_{a b}(x)\right)>0$, that

$$
g(x):=g_{a b}(x) \mathrm{d} x^{a} \otimes \mathrm{d} x^{b},
$$

becomes a Riemannian metric tensor field. Let $\nabla$ be a connection such that $g=\nabla \mathrm{d} \psi$. Then, $x$ is a set of $\nabla$-affine coordinates, $\left\{\Gamma_{a b}^{c}\right\} \equiv 0$, due to

$$
\nabla \mathrm{d} \psi=\left(\frac{\partial^{2} \psi}{\partial x^{a} \partial x^{b}}-\Gamma_{a b}{ }^{c} \frac{\partial \psi}{\partial x^{c}}\right) \mathrm{d} x^{a} \otimes \mathrm{d} x^{b}, \quad \text { where } \quad \nabla \frac{\partial}{\partial x^{a}} \frac{\partial}{\partial x^{b}}=\Gamma_{a b}{ }^{c} \frac{\partial}{\partial x^{c}} .
$$

In addition, it follows from Lemma2.1 that there exists a unique dual connection $\nabla^{*}$ for given $g$ and $\nabla$. It is straightforward to show that $x$ and $p$ are dual coordinates with respect to $g$ :

$$
g\left(\frac{\partial}{\partial x^{a}}, \frac{\partial}{\partial p_{b}}\right)=\delta_{a}^{b}
$$

Applying Lemma2.2, one concludes that $p$ is a set of $\nabla^{*}$-affine coordinates. The connection coefficients for $\nabla^{*}$ such that $\nabla_{\partial / \partial x^{a}}^{*}\left(\partial / \partial x^{b}\right)=\Gamma_{a b}^{*}\left(\partial / \partial x^{c}\right)$ are

$$
\Gamma_{a b}^{* c}=g^{c j} \Gamma_{a b j}^{*}, \quad \text { with } \quad \Gamma_{a b c}^{*}=g\left(\nabla \frac{\partial}{\partial x^{a}} \frac{\partial}{\partial x^{b}}, \frac{\partial}{\partial x^{c}}\right)=\frac{\partial^{3} \psi}{\partial x^{a} \partial x^{b} \partial x^{c}} .
$$

Thus $g, \nabla, \nabla^{*}, \nabla$-affine coordinates, and $\nabla^{*}$-affine coordinates have been obtained.

The following is often used in information geometry.

Definition 2.19. ( Canonical divergence, [14] ) : Let $\left(\mathcal{M}, g, \nabla, \nabla^{*}\right)$ be an $n$-dimensional dually flat space, $\left\{x^{1}, \ldots, x^{n}\right\} \nabla$-affine coordinates, $\left\{p_{1}, \ldots, p_{n}\right\} \nabla^{*}$-affine coordinates, $\xi$ and $\xi^{\prime}$ two points of $\mathcal{M}$. Then, the function $\mathbb{D}: \mathcal{M} \times \mathcal{M} \rightarrow \mathbb{R}$,

$$
\mathbb{D}\left(\xi \| \xi^{\prime}\right):=\psi(\xi)+\varphi\left(\xi^{\prime}\right)-\left.\left.x^{a}\right|_{\xi} p_{a}\right|_{\xi^{\prime}},
$$

is referred to as the canonical divergence.

Remark 2.7. There is another convention for the canonical divergence ( see Ref. [21] ).

Remark 2.8. The canonical divergence (17) should be distinguished from the volume-form divergence that has been in Definition2.2

In information geometry, the following theorem is well-known.

Theorem 2.9. (Generalized Pythagorean theorem, 14]) : Let $\left(\mathcal{M}, g, \nabla, \nabla^{*}\right)$ be a dually flat space, $\mathbb{D}: \mathcal{M} \times \mathcal{M} \rightarrow \mathbb{R}$ the canonical divergence, $\xi^{\prime}, \xi^{\prime \prime}, \xi^{\prime \prime \prime}$ be three points of $\mathcal{M}$ such that 1 . $\xi^{\prime}$ and $\xi^{\prime \prime}$ are connected with the $\nabla^{*}$-geodesic curve and 2. $\xi^{\prime \prime}$ and $\xi^{\prime \prime \prime}$ are connected with the $\nabla$-geodesic curve. Then, it follows that

$$
\mathbb{D}\left(\xi^{\prime \prime \prime} \| \xi^{\prime}\right)=\mathbb{D}\left(\xi^{\prime \prime \prime} \| \xi^{\prime \prime}\right)+\mathbb{D}\left(\xi^{\prime \prime} \| \xi^{\prime}\right)
$$




\section{Contact geometric descriptions of vector fields on dually flat spaces}

In this section, contact geometric descriptions of vector fields on dually flat spaces are shown. These descriptions enable one to have more links between contact geometry and information geometry.

As shown in Theorem 2.8 a contact manifold and a strictly convex function induce a dually flat space. Here points of a dually flat space are identified with ones of the Legendre submanifold generated by the convex function. Since contact Hamiltonian vector fields can describe dynamics on contact manifolds, dynamics on dually flat spaces can also be described by such vector fields.

After fixing mathematical symbols that will be used in Secs 3 such vector fields on dually flat spaces are rewritten in a contact differential geometric language.

\subsection{Mathematical symbols}

In this subsection mathematical symbols are fixed as follows. Let $(\mathcal{C}, \lambda)$ be a $(2 n+1)$-dimensional contact manifold with some fixed $n \in\{1,2, \ldots\},(x, p, z)$ canonical coordinates such that $\lambda=\mathrm{d} z-p_{a} \mathrm{~d} x^{a}$ with $x=\left\{x^{1}, \ldots, x^{n}\right\}$ and $p=\left\{p_{1}, \ldots, p_{n}\right\}, \psi$ a function on $\mathcal{C}$ depending on $x$ only, $\Delta_{0}$ and $\left\{\Delta_{1}, \ldots, \Delta_{n}\right\}$ functions such that

$$
\Delta_{0}(x, z):=\psi(x)-z, \quad \Delta_{a}(x, p):=\frac{\partial \psi}{\partial x^{a}}(x)-p_{a}, \quad(a \in\{1, \ldots, n\})
$$

and ' will denote differential with respect to a parameter denoted $t$.

In addition, to discuss the Legendre duality, other symbols are introduced as follows. Let $\varphi$ be a function on $\mathcal{C}$ depending on $p$ only, and $\Delta^{0}$ and $\left\{\Delta^{1}, \ldots, \Delta^{n}\right\}$ functions such that

$$
\Delta^{0}(x, p, z)=x^{j} p_{j}-\varphi(p)-z, \quad \Delta^{a}(x, p)=x^{a}-\frac{\partial \varphi}{\partial p_{a}}(p), \quad(a \in\{1, \ldots, n\}) .
$$

The following lemma will be used.

Lemma 3.1. ( Equivalent local expressions of Legendre submanifolds ) : The Legendre submanifold $\mathcal{A}_{\psi}$ generated by $\psi$ is expressed such that

$$
\mathcal{A}_{\psi}^{\mathcal{C}}:=\Phi_{\mathcal{C} \mathcal{A} \psi} \mathcal{A}_{\psi}=\left\{(x, p, z) \in \mathcal{C} \mid \Delta_{0}=0, \quad \text { and } \quad \Delta_{1}=\cdots=\Delta_{n}=0\right\},
$$

with $\Phi_{\mathcal{C} \mathcal{A} \psi}: \mathcal{A}_{\psi} \rightarrow \mathcal{C}$ being an embedding. In addition, the Legendre submanifold $\mathcal{A}_{\varphi}$ generated by $-\varphi$ is expressed such that

$$
\mathcal{A}_{\varphi}^{\mathcal{C}}:=\Phi_{\mathcal{C} \mathcal{A} \varphi} \mathcal{A}_{\varphi}=\left\{(x, p, z) \in \mathcal{C} \mid \Delta^{0}=0, \quad \text { and } \quad \Delta^{1}=\cdots=\Delta^{n}=0\right\} .
$$

Proof. For $\mathcal{A}_{\psi}^{\mathcal{C}}$, substituting the definitions of $\Delta_{0},\left\{\Delta_{1}, \ldots, \Delta_{n}\right\}$ into (7), one has (20). For $\mathcal{A}_{\varphi}^{\mathcal{C}}$, one rewrites the conditions in (8). With

$$
x^{a}=\frac{\partial \varphi}{\partial p_{a}} \quad \Longleftrightarrow \quad \Delta^{a}=0
$$

for all $a \in\{1, \ldots, n\}$, substituting $z=p_{j} \partial \varphi / \partial p_{j}-\varphi$ and $x^{a}=\partial \varphi / \partial p_{a}$ into $\Delta^{0}$, one has that

$$
\begin{aligned}
& \left.\Delta^{0}\right|_{z=p_{j} \partial \varphi / \partial p_{j}-\varphi, x^{1}=\partial \varphi / \partial p_{1}, \ldots}=\left(x^{j} p_{j}-\varphi-z\right)_{z=p_{j} \partial \varphi / \partial p_{j}-\varphi, \Delta^{1}=\cdots=\Delta^{n}=0} \\
& =\left(x^{j} p_{j}-\varphi-p_{j} \frac{\partial \varphi}{\partial p_{j}}+\varphi\right)_{\Delta^{1}=\cdots=\Delta^{n}=0}=p_{j}\left(x^{j}-\frac{\partial \varphi}{\partial p_{j}}\right)_{\Delta^{1}=\cdots=\Delta^{n}=0} \\
& =\left(p_{j} \Delta^{j}\right)_{\Delta^{1}=\cdots=\Delta^{n}=0}=0,
\end{aligned}
$$

from which

$$
x^{1}=\frac{\partial \varphi}{\partial p_{1}}, \ldots, x^{n}=\frac{\partial \varphi}{\partial p_{n}}, \quad \text { and } \quad z=p_{i} \frac{\partial \varphi}{\partial p_{i}}-\varphi, \quad \Longrightarrow \quad \Delta^{0}=0, \quad \text { and } \quad \Delta^{1}=\cdots=\Delta^{n}=0 .
$$

In addition, one can easily show that

$$
x^{1}=\frac{\partial \varphi}{\partial p_{1}}, \ldots, x^{n}=\frac{\partial \varphi}{\partial p_{n}}, \quad \text { and } \quad z=p_{i} \frac{\partial \varphi}{\partial p_{i}}-\varphi, \quad \Longleftarrow \quad \Delta^{0}=0, \quad \text { and } \quad \Delta^{1}=\cdots=\Delta^{n}=0 .
$$

Thus one has (21). 


\subsection{Vector fields on dually flat spaces in contact geometric language}

In this subsection, it is shown that vector fields on dually flat spaces can be realized as a class of contact Hamiltonian vector fields with some restriction. For this purpose, functions $\psi$ and $-\varphi$ are strictly convex and the Legendre submanifolds generated by $\psi$ and $-\varphi$ are assumed simply connected in this subsection.

The following proposition can be seen as a variant of the theorem given by Mrugala et al. in Ref. [19].

Proposition 3.1. ( Restricted contact Hamiltonian vector field as the push-forward of a vector field on the Legendre submanifold generated by $\psi)$ : Let $\left\{F_{\psi}^{1}, \ldots, F_{\psi}^{n}\right\}$ be a set of functions of $x$ on $\mathcal{A}_{\psi}$ such that they do not identically vanish, and $\check{X}_{\psi}^{0} \in T_{x} \mathcal{A}_{\psi},\left(x \in \mathcal{A}_{\psi}\right)$ the vector field given as

$$
\check{X}_{\psi}^{0}=\dot{x}^{a} \frac{\partial}{\partial x^{a}}, \quad \text { where } \quad \dot{x}^{a}=F_{\psi}^{a}(x), \quad(a \in\{1, \ldots, n\}) .
$$

In addition, let $X_{\psi}^{0}:=\left(\Phi_{\mathcal{C} \mathcal{A} \psi}\right)_{*} \check{X}_{\psi}^{0} \in T_{\xi} \mathcal{A}_{\psi}^{\mathcal{C}},\left(\xi \in \mathcal{A}_{\psi}^{\mathcal{C}}\right)$ be the push-forward of $\check{X}_{\psi}^{0}$, where $\mathcal{A}_{\psi}^{\mathcal{C}}:=$ $\Phi_{\mathcal{C} \mathcal{A} \psi} \mathcal{A}_{\psi}$ with $\Phi_{\mathcal{C} \mathcal{A} \psi}: \mathcal{A}_{\psi} \rightarrow \mathcal{C}$ being an embedding :

$$
\begin{aligned}
\Phi_{\mathcal{C A} \psi} & : & \mathcal{A}_{\psi} \rightarrow \mathcal{A}_{\psi}^{\mathcal{C}}, & x \mapsto(x, p(x), z(x)) \\
\left(\Phi_{\mathcal{C} \mathcal{A} \psi}\right)_{*} & : & T_{x} \mathcal{A}_{\psi} \rightarrow T_{\xi} \mathcal{A}_{\psi}^{\mathcal{C}}, & \check{X}_{\psi}^{0} \mapsto X_{\psi}^{0} .
\end{aligned}
$$

Then it follows that

$$
X_{\psi}^{0}=\dot{x}^{a} \frac{\partial}{\partial x^{a}}+\dot{p}_{a} \frac{\partial}{\partial p_{a}}+\dot{z} \frac{\partial}{\partial z}, \quad \text { where } \quad \dot{x}^{a}=F_{\psi}^{a}(x), \quad \dot{p}_{a}=\frac{\mathrm{d}}{\mathrm{d} t}\left(\frac{\partial \psi}{\partial x^{a}}\right), \quad \dot{z}=\frac{\mathrm{d} \psi}{\mathrm{d} t} .
$$

In addition, one has that $X_{\psi}^{0}=\left.X_{h_{\psi}}\right|_{h_{\psi}=0}$. Here $X_{h_{\psi}}$ is the contact Hamiltonian flow associated to

$$
h_{\psi}(x, p, z)=\Delta_{a}(x, p) F_{\psi}^{a}(x)+\Gamma_{\psi}\left(\Delta_{0}(x, z)\right),
$$

where $\Gamma_{\psi}$ is a function of $\Delta_{0}$ such that $\Gamma_{\psi}(0)=0$ and $\Gamma_{\psi}\left(\Delta_{0}\right) \neq 0$ for $\Delta_{0} \neq 0$.

Proof. To show (22), one has

$$
\begin{aligned}
X_{\psi}^{0} & =\left(\Phi_{\mathcal{C A} \psi}\right)_{*} \check{X}_{\psi}^{0}=\dot{x}^{a} \frac{\partial}{\partial x^{a}}+\dot{x}^{a} \frac{\partial p_{b}}{\partial x^{a}} \frac{\partial}{\partial p_{b}}+\dot{x}^{a} \frac{\partial z}{\partial x^{a}} \frac{\partial}{\partial z}=\dot{x}^{a} \frac{\partial}{\partial x^{a}}+\left(\frac{\mathrm{d}}{\mathrm{d} t} \frac{\partial \psi}{\partial x^{a}}\right) \frac{\partial}{\partial p_{a}}+\frac{\mathrm{d} \psi}{\mathrm{d} t} \frac{\partial}{\partial z} \\
& =\dot{x}^{a} \frac{\partial}{\partial x^{a}}+\dot{p}_{a} \frac{\partial}{\partial p_{a}}+\dot{z} \frac{\partial}{\partial z}
\end{aligned}
$$

from which one has (22).

In the following, it is shown that $X_{\psi}^{0}=\left.X_{h_{\psi}}\right|_{h_{\psi}=0}$ with $h_{\psi}$ given by (23). First, one has the component expression of the contact Hamiltonian vector field associated to the contact Hamiltonian (23) without any restriction as

$$
X_{h_{\psi}}=\dot{x}^{a} \frac{\partial}{\partial x^{a}}+\dot{p}_{a} \frac{\partial}{\partial p_{a}}+\dot{z} \frac{\partial}{\partial z},
$$

where

$$
\frac{\mathrm{d} x^{a}}{\mathrm{~d} t}=F_{\psi}^{a}, \quad \frac{\mathrm{d} p_{a}}{\mathrm{~d} t}=\frac{\partial^{2} \psi}{\partial x^{a} \partial x^{b}} F_{\psi}^{b}+\left(\frac{\partial \psi}{\partial x^{b}}-p_{b}\right) \frac{\partial F_{\psi}^{b}}{\partial x^{a}}+\left(\frac{\partial \psi}{\partial x^{a}}-p_{a}\right) \frac{\mathrm{d} \Gamma_{\psi}}{\mathrm{d} \Delta_{0}}, \quad \frac{\mathrm{d} z}{\mathrm{~d} t}=h_{\psi}^{0}+p_{a} F_{\psi}^{a} .
$$

Second, observe that the condition $h_{\psi}=\Delta_{a} F_{\psi}^{a}+\Gamma_{\psi}\left(\Delta_{0}\right)=0$ is equivalent to that $\Delta_{0}=\cdots=\Delta_{n}=0$, since $\left\{F_{\psi}^{1}, \ldots, F_{\psi}^{n}\right\}$ do not identically vanish and the property of $\Gamma_{\psi}$. From this and (20), one has that $\mathcal{A}_{\psi}^{\mathcal{C}}=\left\{h_{\psi}=0\right\}$. Finally, the contact Hamiltonian vector field restricted to $h_{\psi}^{0}=0$ is obtained by substituting $\Delta_{0}=\cdots=\Delta_{n}=0$ into $X_{h_{\psi}}$ as

$$
\frac{\mathrm{d} x^{a}}{\mathrm{~d} t}=F_{\psi}^{a}, \quad \frac{\mathrm{d} p_{a}}{\mathrm{~d} t}=\frac{\partial^{2} \psi}{\partial x^{a} \partial x^{b}} F_{\psi}^{b}=\frac{\partial^{2} \psi}{\partial x^{a} \partial x^{b}} \frac{\mathrm{d} x^{b}}{\mathrm{~d} t}, \quad \frac{\mathrm{d} z}{\mathrm{~d} t}=p_{a} F_{\psi}^{a}=\frac{\partial \psi}{\partial x^{a}} \frac{\mathrm{d} x^{a}}{\mathrm{~d} t},
$$

which are equivalent to (22). Thus, one has that $X_{\psi}^{0}=\left.X_{h_{\psi}}\right|_{h_{\psi}=0}$. 
Remark 3.1. The functions $\left\{F_{\psi}^{1}, \ldots, F_{\psi}^{n}\right\}$ need not depend on $\psi$.

Remark 3.2. The value of the generating function $\psi$ is not conserved along this restricted contact Hamiltonian vector field, since

$$
\mathcal{L}_{X_{\psi}^{0}} \psi=\frac{\partial \psi}{\partial x^{a}} \frac{\mathrm{d} x^{a}}{\mathrm{~d} t}=\frac{\partial \psi}{\partial x^{a}} F_{\psi}^{a},
$$

does not identically vanish in general. In some cases, this vanishes. Consider the system with $n=2$, and $F_{\psi}^{1}=\partial \psi / \partial x^{2}, F_{\psi}^{2}=-\partial \psi / \partial x^{1}$. Then $\mathcal{L}_{X_{\psi}^{0}} \psi=0$.

Remark 3.3. In the case of $\Gamma_{\psi} \equiv 0$, this contact Hamiltonian has been used in Ref. [16], and it follows from (11) that $\mathcal{L}_{X_{\psi}^{0}} \lambda=0$.

The following is a counterpart of Proposition 3.1 .

Proposition 3.2. ( Restricted contact Hamiltonian vector field as the push-forward of vector fields on the Legendre submanifold generated by $-\varphi):$ Let $\left\{F_{1}^{\varphi}, \ldots, F_{n}^{\varphi}\right\}$ be a set of functions of $p$ on $\mathcal{A}_{\varphi}$ such that they do not identically vanish, and $\check{X}_{\varphi}^{0} \in T_{p} \mathcal{A}_{\varphi},\left(p \in \mathcal{A}_{\varphi}\right)$ given as

$$
\check{X}_{\varphi}^{0}=\dot{p}_{a} \frac{\partial}{\partial p_{a}}, \quad \text { where } \dot{p}_{a}=F_{a}^{\varphi}(p)
$$

In addition, let $X_{\varphi}^{0}:=\left(\Phi_{\mathcal{C A} \varphi}\right)_{*} \check{X}_{\varphi}^{0} \in T_{\xi} \mathcal{A}_{\varphi}^{\mathcal{C}},\left(\xi \in \mathcal{A}_{\varphi}^{\mathcal{C}}\right)$ be the push-forward of $\check{X}_{\varphi}^{0}$, where $\mathcal{A}_{\varphi}^{\mathcal{C}}:=$ $\Phi_{\mathcal{C A} \varphi} \mathcal{A}_{\varphi}$ with $\Phi_{\mathcal{C A} \varphi}: \mathcal{A}_{\varphi} \rightarrow \mathcal{C}$ being an embedding :

$$
\begin{aligned}
\Phi_{\mathcal{C A} \varphi} & : \mathcal{A}_{\varphi} \rightarrow \mathcal{A}_{\varphi}^{\mathcal{C}}, & & x \mapsto(x(p), p, z(p)) \\
\left(\Phi_{\mathcal{C} \mathcal{A} \varphi}\right)_{*} & : & T_{p} \mathcal{A}_{\varphi} \rightarrow T_{\xi} \mathcal{A}_{\varphi}^{\mathcal{C}}, & \check{X}_{\varphi}^{0} \mapsto X_{\varphi}^{0} .
\end{aligned}
$$

Then it follows that

$$
X_{\varphi}^{0}=\dot{x}^{a} \frac{\partial}{\partial x^{a}}+\dot{p}_{a} \frac{\partial}{\partial p_{a}}+\dot{z} \frac{\partial}{\partial z}, \quad \text { where } \quad \dot{x}_{a}=\frac{\mathrm{d}}{\mathrm{d} t}\left(\frac{\partial \varphi}{\partial p_{a}}\right), \quad \dot{p}^{a}=F_{a}^{\varphi}(p), \quad \dot{z}=p_{j} F_{k}^{\varphi} \frac{\partial^{2} \varphi}{\partial p_{k} \partial p_{j}}
$$

In addition, one has that $X_{\varphi}^{0}=\left.X_{h_{\varphi}}\right|_{h_{\varphi}=0}$. Here $X_{h_{\varphi}}$ is the contact Hamiltonian associated to

$$
h_{\varphi}(x, p)=\Delta^{a}(x, p) F_{a}^{\varphi}(p)+\Gamma^{\varphi}\left(\Delta^{0}\right),
$$

where $\Gamma^{\varphi}$ is a function of $\Delta^{0}$ such that $\Gamma^{\varphi}(0)=0$ and $\Gamma^{0}\left(\Delta^{0}\right) \neq 0$ for $\Delta^{0} \neq 0$.

Proof. A proof is analogous to the proof of Proposition 3.1

Remark 3.4. The functions $\left\{F_{1}^{\varphi}, \ldots, F_{n}^{\varphi}\right\}$ need not depend on $\varphi$.

Remark 3.5. The value of the generating function $\varphi$ is not conserved along this restricted contact Hamiltonian vector field, since

$$
\mathcal{L}_{X_{\varphi}^{0}} \varphi=\frac{\partial \varphi}{\partial p_{a}} \frac{\mathrm{d} p_{a}}{\mathrm{~d} t}=\frac{\partial \varphi}{\partial p_{a}} F_{a}^{\varphi},
$$

does not identically vanish in general. In some cases, this vanishes. Consider the system with $n=2$, and $F_{1}^{\varphi}=\partial \varphi / \partial p_{2}, F_{2}^{\varphi}=-\partial \varphi / \partial p_{1}$. Then $\mathcal{L}_{X_{\varphi}^{0}} \varphi=0$.

Remark 3.6. In the case of $\Gamma^{\varphi} \equiv 0$, it follows from (11) that $\mathcal{L}_{X}{ }_{\varphi} \lambda=0$.

In $\$ 5$ another formulation will be introduced. In that formulation the value of the generating functions are conserved along contact Hamiltonian vector fields for any $\left\{F_{\psi}^{1}, \ldots, F_{\psi}^{n}\right\}$. In addition its counterpart will also be given.

In general, when connections are given on a manifold, one of the most important geometric objects is a set of geodesic curves. On a dually flat space, two connections are given and then two kinds of geodesic curves are of interest. In the following, it is shown that such geodesic curves are realized by the integral curves of the contact Hamiltonian vector fields by choosing $\left\{F_{\psi}^{1}, \ldots, F_{\psi}^{n}\right\}$ in (22) and $\left\{F_{1}^{\varphi}, \ldots, F_{n}^{\varphi}\right\}$ in 24. 
Theorem 3.1. ( $\nabla^{*}$-geodesic curves ) : Let $p^{\prime}=\left\{p_{1}^{\prime}, \ldots, p_{n}^{\prime}\right\}$ and $p^{\prime \prime}=\left\{p_{1}^{\prime \prime}, \ldots, p_{n}^{\prime \prime}\right\}$ be two sets of constants, $\psi$ a strictly convex function of $x$ only, $\left\{g^{a b}(x)\right\}$ the metric components (16) where the arguments are written in terms of $x$. Then the restricted contact Hamiltonian vector field associated to $h_{\psi}$ in (23) with

$$
F_{\psi}^{a}(x)=g^{a j}(x)\left(p_{j}^{\prime \prime}-p_{j}^{\prime}\right), \quad(a \in\{1, \ldots, n\})
$$

gives the geodesic curve connecting the two points whose $\nabla^{*}$-affine coordinates are $p^{\prime}$ and $p^{\prime \prime}$.

Proof. It follows from (22) that

$$
\frac{\mathrm{d}}{\mathrm{d} t} p_{a}=g_{a j} \frac{\mathrm{d} x^{j}}{\mathrm{~d} t}=g_{a j} F_{\psi}^{j}=g_{a j} g^{j k}\left(p_{k}^{\prime \prime}-p_{k}^{\prime}\right)=p_{a}^{\prime \prime}-p_{a}^{\prime} .
$$

The solution to this equation is obtained as

$$
p_{a}(t)=\left(p_{a}^{\prime \prime}-p_{a}^{\prime}\right) t+p_{a}(0) .
$$

Choosing the initial point $p(0)$ as $p^{\prime}$, one has the straight line that connects $p^{\prime}$ and $p^{\prime \prime}$. This line is the geodesic curve in this case, and connects the two points whose $\nabla^{*}$-affine coordinates are $p^{\prime}$ and $p^{\prime \prime}$, respectively.

Rather than $p(t)$ in the theorem, one way to express $x(t)$ analytically is to use the relation $x^{a}=$ $\partial \varphi / \partial p_{a}$, where the analytic expression of $\varphi=\mathfrak{L}[\psi]$ is required. Although such an explicit expression cannot be obtained in general, there are some cases where it is feasible. In Ref. [20, the explicit analytic forms of the total Legendre transform of given strictly convex functions and their applications in linear programming problems have been studied.

There is a counterpart as follows.

Theorem 3.2. ( $\nabla$-geodesic curves ) : Let $x^{\prime}=\left\{x^{\prime 1}, \ldots, x^{\prime n}\right\}$ and $x^{\prime \prime}=\left\{x^{\prime \prime 1}, \ldots, x^{\prime \prime n}\right\}$ be constants, $\varphi$ a strictly convex function of $p$ only, $\left\{g_{a b}(p)\right\}$ the metric components (16) where the arguments are written in terms of $p$. Then the restricted contact Hamiltonian vector field associated to $h_{\varphi}$ in (25) with

$$
F_{a}^{\varphi}(p)=g_{a j}(p)\left(x^{\prime \prime j}-x^{\prime j}\right), \quad(a \in\{1, \ldots, n\})
$$

gives the geodesic curve connecting the two points whose $\nabla$-affine coordinates are $x^{\prime}$ and $x^{\prime \prime}$.

Proof. A way to prove this is analogous to that of Theorem 3.1

Similar to Theorems 3.1] one can write Theorem 1 of Ref. 21] ( see also Ref. [22] ) in terms of a contact Hamiltonian vector field. Note that the definition of the canonical divergence in Ref. [21] is not same as that of this paper.

Theorem 3.3. (Gradient flow of Ref. [21] in terms of a restricted contact Hamiltonian vector field) $:$ Let $\psi$ be a strictly convex function of $x$ only, $\xi \in \mathcal{A}_{\psi}^{\mathcal{C}}$ a point whose coordinates are $(x, p(x), z(x))$, $\xi^{\prime} \in \mathcal{A}_{\psi}^{\mathcal{C}}$ another point, and $\left\{g^{a b}(x)\right\}$ given by (16) with the arguments rewritten in terms of $x$. Choose $\left\{F_{\psi}^{1}, \ldots, F_{\psi}^{n}\right\}$ in (22) as

$$
F_{\psi}^{a}(x)=-g^{a j}(x) \frac{\partial}{\partial x^{j}} \mathbb{D}\left(\xi \| \xi^{\prime}\right) .
$$

Then, the flow converges to the point $\xi^{\prime}$ along the $\nabla^{*}$-geodesic curve. This flow is the restricted contact Hamiltonian vector field $\left.X_{h_{\psi}}\right|_{h_{\psi}=0}$ associated to $h_{\psi}$ given in (23) with this set of functions $\left\{F_{\psi}^{1}, \ldots, F_{\psi}^{n}\right\}$.

Proof. A proof is similar to that for Theorems 3.1] ( see also Ref. 21] ). Let $\left(x^{\prime}, p^{\prime}, z^{\prime}\right)$ be coordinates for $\xi^{\prime}$. It follows from (22) and (17) that

$$
\begin{aligned}
\frac{\mathrm{d}}{\mathrm{d} t} p_{a} & =g_{a j} \frac{\mathrm{d} x^{j}}{\mathrm{~d} t}=-g_{a j} g^{j k}(x) \frac{\partial}{\partial x^{k}} \mathbb{D}\left(\xi \| \xi^{\prime}\right) .=-\delta_{a}^{k} \frac{\partial}{\partial x^{k}}\left[\psi(x)+\varphi\left(p^{\prime}\right)-x^{j} p_{j}^{\prime}\right] \\
& =-\frac{\partial}{\partial x^{a}}\left[\psi(x)-x^{j} p_{j}^{\prime}\right]=-\left(p_{a}-p_{a}^{\prime}\right),
\end{aligned}
$$

which is integrated to obtain

$$
p_{a}(t)=p_{a}^{\prime}+\left(p_{a}(0)-p_{a}^{\prime}\right) \mathrm{e}^{-t} .
$$

This and Proposition 3.1 prove the theorem. 
Then, one has a counterpart of Theorem 3.3 as follows.

Theorem 3.4. ( Counterpart of the gradient flow of Ref. [21] in terms of a restricted contact Hamiltonian vector field ) : Let $\varphi$ be a strictly convex function of $p$ only, $\xi \in \mathcal{A}_{\varphi}^{\mathcal{C}}$ a point whose coordinates are $(x(p), p, z(p)), \xi^{\prime} \in \mathcal{A}_{\varphi}^{\mathcal{C}}$ another point, and $\left\{g_{a b}(p)\right\}$ given by (16) with the arguments being rewritten in terms of $p$. Choose $\left\{F_{1}^{\varphi}, \ldots, F_{n}^{\varphi}\right\}$ in 24) to be

$$
F_{a}^{\varphi}(p)=-g_{a j}(p) \frac{\partial}{\partial p_{j}} \mathbb{D}\left(\xi^{\prime} \| \xi\right) .
$$

Then, the flow converges to the point $\xi^{\prime}$ along the $\nabla$-geodesic curve. This flow is the restricted contact Hamiltonian vector field $\left.X_{h_{\varphi}}\right|_{h_{\varphi}=0}$ associated to $h_{\varphi}$ given in (25) with this set of functions $\left\{F_{1}^{\varphi}, \ldots, F_{n}^{\varphi}\right\}$.

Proof. A way to prove this is similar to that of Theorems 3.3 ( see also Ref. 21] ).

With Theorems 3.1 and 3.2. the generalized Pythagorean theorem ( Theorem 2.9) is written in terms of two contact Hamiltonian vector fields as follows.

Theorem 3.5. (Generalized Pythagorean theorem in terms of integral curves ) : Let $\psi$ be a strictly convex function depending on $x$ only, $\varphi$ the function of $p$ that is obtained by the total Legendre transform of $\psi$ with respect to $x$, and $\xi^{\prime}, \xi^{\prime \prime}, \xi^{\prime \prime \prime}$ three points of $\mathcal{A}_{\psi}^{\mathcal{C}} \cap \mathcal{A}_{\varphi}^{\mathcal{C}}$ such that

1. $\xi^{\prime}$ and $\xi^{\prime \prime}$ are connected with the $\nabla^{*}$-geodesic curve, and any point of the $\nabla^{*}$-geodesic curve is on $\mathcal{A}_{\psi}^{\mathcal{C}} \cap \mathcal{A}_{\varphi}^{\mathcal{C}}$

2. $\xi^{\prime \prime}$ and $\xi^{\prime \prime \prime}$ are connected with the $\nabla$-geodesic curve, and any point of the $\nabla$-geodesic curve is on $\mathcal{A}_{\psi}^{\mathcal{C}} \cap \mathcal{A}_{\varphi}^{\mathcal{C}}$.

Then the generalized Pythagorean theorem ( Theorem 2.9) can be written as

$$
\mathbb{D}\left(\operatorname{Exp}\left(X_{\varphi}^{0}\right) \operatorname{Exp}\left(X_{\psi}^{0}\right) \xi^{\prime} \| \xi^{\prime}\right)=\mathbb{D}\left(\operatorname{Exp}\left(X_{\varphi}^{0}\right) \xi^{\prime \prime} \| \xi^{\prime \prime}\right)+\mathbb{D}\left(\operatorname{Exp}\left(X_{\psi}^{0}\right) \xi^{\prime} \| \xi^{\prime}\right)
$$

and

$$
\mathbb{D}\left(\operatorname{Exp}\left(X_{\varphi}^{0}\right) \operatorname{Exp}\left(X_{\psi}^{0}\right) \xi^{\prime} \| \xi^{\prime}\right)=\mathbb{D}\left(\operatorname{Exp}\left(X_{\varphi}^{0}\right) \operatorname{Exp}\left(X_{\psi}^{0}\right) \xi^{\prime} \| \operatorname{Exp}\left(X_{\psi}^{0}\right) \xi^{\prime}\right)+\mathbb{D}\left(\operatorname{Exp}\left(X_{\psi}^{0}\right) \xi^{\prime} \| \xi^{\prime}\right)
$$

where $\mathbb{D}$ is the canonical divergence defined in 17$), \operatorname{Exp}\left(X_{\psi}^{0}\right): \mathcal{A}_{\psi}^{\mathcal{C}} \rightarrow \mathcal{A}_{\psi}^{\mathcal{C}}$ is the exponential map of unit time generated by the flow (22) with (26) and $\operatorname{Exp}\left(X_{\varphi}^{0}\right): \mathcal{A}_{\varphi}^{\mathcal{C}} \rightarrow \mathcal{A}_{\varphi}^{\mathcal{C}}$ is the one generated by the flow 24) with 27.

Proof. Applying Theorem[2.9, Theorem 3.1, and Theorem 3.2 one completes the proof.

\subsection{Applications to electric circuit models}

In this subsection, it is shown how the developed general theory is applied to engineering problems. As examples, three simple electric circuit models are analyzed. The first one is a series RC ( resistorcapacitor ) circuit model. The second one is a series RL ( resistor-inductor ) circuit model. These ones are formulated on 1-dimensional dually flat spaces. The third one is a series RLC ( resistor-inductorcapacitor ) circuit model. This one is formulated on a 2-dimensional dually flat space. For each model, after defining the circuit model, it is shown how the model can be seen as a dynamical system on a dually flat space embedded in a contact manifold.

First, a circuit model consisting of a resistor and a capacitor is focused.

Definition 3.1. ( $R C$ circuit model ) : Let $C$ be a constant capacitor with $C>0, R$ a constant resistor with $R>0, Q_{\mathrm{C}} \in \mathbb{R}$ the amount of charge, $V_{\mathrm{C}} \in \mathbb{R}$ the capacitor voltage, $\mathcal{H}_{\mathrm{C}}\left(Q_{\mathrm{C}}\right) \in \mathbb{R}$ the energy stored in the capacitor, and $t \in \mathbb{R}$ time. The $R C$ circuit model is defined as the following system

$$
R \frac{\mathrm{d} Q_{\mathrm{C}}}{\mathrm{d} t}=-\frac{1}{C} Q_{\mathrm{C}}
$$

together with

$$
\mathcal{H}_{\mathrm{C}}\left(Q_{\mathrm{C}}\right):=\frac{1}{2 C} Q_{\mathrm{C}}^{2}, \quad \text { and } \quad Q_{\mathrm{C}}=C V_{\mathrm{C}}
$$


Remark 3.7. The system (28) with (29) can be seen as an equation for describing electric energy dissipation due to the existence of the resistor.

Theorem 3.6. ( $R C$ circuit model on a dually flat space) : The system (28) can be seen as a dynamical system on a dually flat space. In particular, the coordinate systems $Q_{\mathrm{C}}$ and $V_{\mathrm{C}}$ can be seen as being mutually dual with respect to a metric tensor field.

Proof. Since $\mathcal{H}_{\mathrm{C}}$ is a strictly convex function on $\mathbb{R}$ whose coordinate is $Q_{\mathrm{C}}$, one can identify d $\mathcal{H}_{\mathrm{C}} / \mathrm{d} Q_{\mathrm{C}}^{2}$ as the component of a Riemannian metric tensor field $g_{\mathrm{C}}$. These are summarized as

$$
g_{\mathrm{C}}:=g_{\mathrm{C} 11} \mathrm{~d} Q_{\mathrm{C}} \otimes \mathrm{d} Q_{\mathrm{C}}, \quad g_{\mathrm{C} 11}:=\frac{\mathrm{d}^{2} \mathcal{H}_{\mathrm{C}}}{\mathrm{d} Q_{\mathrm{C}}^{2}}=\frac{1}{C}>0 .
$$

Defining the total Legendre transform of $\mathcal{H}_{\mathrm{C}}$ with respect to $Q_{\mathrm{C}}$ as

$$
\mathcal{H}_{\mathrm{C}}^{*}\left(V_{\mathrm{C}}\right):=\sup _{Q_{\mathrm{C}}}\left(Q_{\mathrm{C}} V_{\mathrm{C}}-\mathcal{H}_{\mathrm{C}}\left(Q_{\mathrm{C}}\right)\right)
$$

one has that

$$
\mathcal{H}_{\mathrm{C}}^{*}\left(V_{\mathrm{C}}\right)=\frac{C}{2} V_{\mathrm{C}}^{2}
$$

One can write $g_{\mathrm{C}}$ as

$$
g_{\mathrm{C}}=g_{\mathrm{C} 11}^{-1} \mathrm{~d} V_{\mathrm{C}} \otimes \mathrm{d} V_{\mathrm{C}}, \quad g_{\mathrm{C} 11}^{-1}:=\frac{\mathrm{d}^{2} \mathcal{H}_{\mathrm{C}}^{*}}{\mathrm{~d} V_{\mathrm{C}}^{2}}=C>0 .
$$

It follows from

$$
g_{\mathrm{C} 11} g_{\mathrm{C} 11}^{-1}=1, \quad Q_{\mathrm{C}}=\frac{\mathrm{d} \mathcal{H}_{\mathrm{C}}^{*}}{\mathrm{~d} V_{\mathrm{C}}}, \quad V_{\mathrm{C}}=\frac{\mathrm{d} \mathcal{H}_{\mathrm{C}}}{\mathrm{d} Q_{\mathrm{C}}}, \quad g_{\mathrm{C} 11}^{-1}=\frac{\mathrm{d}^{2} \mathcal{H}_{\mathrm{C}}^{*}}{\mathrm{~d} V_{\mathrm{C}}^{2}}=\frac{\mathrm{d} Q_{\mathrm{C}}}{\mathrm{d} V_{\mathrm{C}}}, \quad g_{\mathrm{C} 11}=\frac{\mathrm{d}^{2} \mathcal{H}_{\mathrm{C}}}{\mathrm{d} Q_{\mathrm{C}}^{2}}=\frac{\mathrm{d} V_{\mathrm{C}}}{\mathrm{d} Q_{\mathrm{C}}},
$$

that

$$
g_{\mathrm{C}}\left(\frac{\partial}{\partial Q_{\mathrm{C}}}, \frac{\partial}{\partial V_{\mathrm{C}}}\right)=1 \text {. }
$$

Therefore, $Q_{\mathrm{C}}$ and $V_{\mathrm{C}}$ are mutually dual with respect to $g_{\mathrm{C}}$ ( see Definition2.17).

The pair $\left(\mathbb{R}, g_{\mathrm{C}}\right)$ is a Riemannian manifold. Let $\nabla_{\mathrm{C}}$ be the connection such that $g_{\mathrm{C}}=\nabla_{\mathrm{C}} \mathrm{d} \mathcal{H}_{\mathrm{C}}$. Then, $Q_{\mathrm{C}}$ is the $\nabla_{\mathrm{C}}$-affine coordinate, and there exists the unique dual connection $\nabla_{\mathrm{C}}^{*}$ due to Lemma 2.1 Applying Lemma2.2 with $Q_{\mathrm{C}}$ and $V_{\mathrm{C}}$ being mutually dual with respect to $g_{\mathrm{C}}$, one has that $\left(\mathbb{R}, g_{\mathrm{C}}, \nabla_{\mathrm{C}}, \nabla_{\mathrm{C}}^{*}\right.$ ) is a 1-dimensional dually flat space ( see Definition2.18). In addition, (28) can be seen as a dynamical system on a 1-dimensional dually flat space.

In what follows the dynamical system (28) with the condition $Q_{\mathrm{C}}=C V_{\mathrm{C}}$ in (29) is identified with a vector field on the embedded Legendre submanifold generated by $\mathcal{H}_{\mathrm{C}}$ defined in (29).

Proposition 3.3. ( $R C$ circuit model as a dynamical system on a Legendre submanifold ) : Let $\left(\mathcal{C}_{\mathrm{C}}, \lambda_{\mathrm{C}}\right)$ be a contact manifold with $\mathcal{C}_{\mathrm{C}}=\mathbb{R}^{3},\left(Q_{\mathrm{C}}, V_{\mathrm{C}}, z_{\mathrm{C}}\right)$ coordinates of $\mathcal{C}_{\mathrm{C}}$, and $\lambda_{\mathrm{C}}=\mathrm{d} z_{\mathrm{C}}-V_{\mathrm{C}} \mathrm{d} Q_{\mathrm{C}}$. Then, the vector field on $\mathcal{C}_{\mathrm{C}}$ associated with (28) together with (29) is on the Legendre submanifold generated by $\mathcal{H}_{\mathrm{C}}$, and its component expressions are

$$
\frac{\mathrm{d}}{\mathrm{d} t} Q_{\mathrm{C}}=-\frac{1}{R C} Q_{\mathrm{C}}, \quad \frac{\mathrm{d}}{\mathrm{d} t} V_{\mathrm{C}}=-\frac{1}{R C^{2}} Q_{\mathrm{C}}, \quad \frac{\mathrm{d}}{\mathrm{d} t} z_{\mathrm{C}}=-\frac{1}{R C^{2}} Q_{\mathrm{C}}^{2},
$$

together with the conditions

$$
Q_{C}=C V_{\mathrm{C}}, \quad \text { and } \quad z_{\mathrm{C}}=\frac{1}{2 C} Q_{\mathrm{C}}^{2} .
$$

Proof. Let $F_{\mathrm{C}}$ be the following function of $Q_{\mathrm{C}}$

$$
F_{\mathrm{C}}\left(Q_{\mathrm{C}}\right)=-\frac{1}{R C} Q_{\mathrm{C}}
$$

so that (28) can be written as

$$
\frac{\mathrm{d}}{\mathrm{d} t} Q_{\mathrm{C}}=F_{\mathrm{C}}\left(Q_{\mathrm{C}}\right)
$$


Applying Proposition 3.1 one has that

$$
\frac{\mathrm{d}}{\mathrm{d} t} Q_{\mathrm{C}}=F_{\mathrm{C}}\left(Q_{\mathrm{C}}\right)=-\frac{Q_{\mathrm{C}}}{R C}, \quad \frac{\mathrm{d}}{\mathrm{d} t} V_{\mathrm{C}}=\frac{\mathrm{d}}{\mathrm{d} t} \frac{\mathrm{d} \mathcal{H}_{\mathrm{C}}}{\mathrm{d} Q_{\mathrm{C}}}=-\frac{Q_{\mathrm{C}}}{R C^{2}}, \quad \frac{\mathrm{d}}{\mathrm{d} t} z_{\mathrm{C}}=\frac{\mathrm{d} \mathcal{H}_{\mathrm{C}}}{\mathrm{d} t}=-\frac{Q_{\mathrm{C}}^{2}}{R C^{2}} .
$$

The vector field associated with this dynamical system is on the Legendre submanifold, where the following relations

$$
\begin{aligned}
& \Delta_{0}^{\mathrm{C}}\left(Q_{\mathrm{C}}, z_{\mathrm{C}}\right):=\mathcal{H}_{\mathrm{C}}-z_{\mathrm{C}}=\frac{Q_{\mathrm{C}}^{2}}{2 C}-z_{\mathrm{C}}=0, \\
& \Delta_{1}^{\mathrm{C}}\left(Q_{\mathrm{C}}, V_{\mathrm{C}}\right):=\frac{\mathrm{d} \mathcal{H}_{\mathrm{C}}}{\mathrm{d} Q_{\mathrm{C}}}-V_{\mathrm{C}}=\frac{Q_{\mathrm{C}}}{C}-V_{\mathrm{C}}=0,
\end{aligned}
$$

hold. From these relations, one has that $Q_{\mathrm{C}}=C V_{\mathrm{C}}$ and $z_{\mathrm{C}}=Q_{\mathrm{C}}^{2} /(2 C)$. In addition, this vector field is written as $\left.X_{h_{\mathrm{C}}}\right|_{h_{\mathrm{C}}=0}$, where $X_{h_{\mathrm{C}}}$ is the contact Hamiltonian vector field associated to $h_{\mathrm{C}}\left(Q_{\mathrm{C}}, V_{\mathrm{C}}, z_{\mathrm{C}}\right)=\Delta_{1}^{\mathrm{C}}\left(Q_{\mathrm{C}}, z_{\mathrm{C}}\right) F_{\mathrm{C}}\left(Q_{\mathrm{C}}\right)+\Gamma_{\mathrm{C}}\left(\Delta_{0}^{\mathrm{C}}\right)$, with $\Gamma_{\mathrm{C}}$ being a function such that $\Gamma_{\mathrm{C}}(0)=0$ and $\Gamma_{\mathrm{C}}\left(\Delta_{0}^{\mathrm{C}}\right) \neq 0$ for $\Delta_{0}^{\mathrm{C}} \neq 0$. The condition $h_{\mathrm{C}}=0$ is equivalent to the conditions, $\Delta_{0}^{\mathrm{C}}=0$ and $\Delta_{1}^{\mathrm{C}}=0$.

Remark 3.8. The discussions above on the RC circuit model can be extended to those on a class where the energy stored in the capacitor

$$
\mathcal{H}_{\mathrm{C}}\left(Q_{\mathrm{C}}\right)=\int Q_{\mathrm{C}}\left(V_{\mathrm{C}}\right) \mathrm{d} V_{\mathrm{C}}
$$

is a strictly convex function of $Q_{\mathrm{C}}$ with $Q_{\mathrm{C}}=Q_{\mathrm{C}}\left(V_{\mathrm{C}}\right)$ being not necessary to be $Q_{\mathrm{C}}=C V_{\mathrm{C}}$.

Second, a circuit model consisting of a resistor and an inductor is focused.

Definition 3.2. ( $R L$ circuit model ) : Let $L$ be a constant inductor with $L>0, R$ a constant resistor with $R>0, I_{\mathrm{L}} \in \mathbb{R}$ the current, $N_{\mathrm{L}} \in \mathbb{R}$ magnetic flux due to $I_{\mathrm{L}}, \mathcal{H}_{\mathrm{L}}^{*}\left(I_{\mathrm{L}}\right) \in \mathbb{R}$ the magnetic energy, and $t \in \mathbb{R}$ time. The $R L$ circuit model is defined as the following system

$$
L \frac{\mathrm{d}}{\mathrm{d} t} I_{\mathrm{L}}=-R I_{\mathrm{L}}
$$

together with

$$
\mathcal{H}_{\mathrm{L}}^{*}\left(I_{\mathrm{L}}\right):=\frac{L}{2} I_{\mathrm{L}}^{2}, \quad \text { and } \quad N_{\mathrm{L}}=L I_{\mathrm{L}} .
$$

Remark 3.9. The system (31) with (32) can be seen as an equation for describing magnetic energy dissipation due to the existence of the resistor.

Theorem 3.7. ( RL circuit model on a dually flat space) : The system (31) can be seen as a dynamical system on a dually flat space. In particular, the coordinate systems $N_{\mathrm{L}}$ and $I_{\mathrm{L}}$ can be seen as being mutually dual with respect to a metric tensor field.

Proof. Since $\mathcal{H}_{\mathrm{L}}^{*}$ is a strictly convex function on $\mathbb{R}$ whose coordinate is $I_{\mathrm{L}}$, one can identify $\mathrm{d}^{2} \mathcal{H}_{\mathrm{L}}^{*} / \mathrm{d} I_{\mathrm{L}}^{2}$ as the component of a Riemannian metric tensor field $g_{\mathrm{L}}$. These are summarized as

$$
g_{\mathrm{L}}:=g_{\mathrm{L} 11}^{-1} \mathrm{~d} I_{\mathrm{L}} \otimes \mathrm{d} I_{\mathrm{L}}, \quad g_{\mathrm{L} 11}^{-1}:=\frac{\mathrm{d}^{2} \mathcal{H}_{\mathrm{L}}^{*}}{\mathrm{~d} I_{\mathrm{L}}^{2}}=L>0 .
$$

Defining the total Legendre transform of $\mathcal{H}_{\mathrm{L}}^{*}$ with respect to $I_{\mathrm{L}}$ as

$$
\mathcal{H}_{\mathrm{L}}\left(N_{\mathrm{L}}\right):=\sup _{I_{\mathrm{L}}}\left(I_{\mathrm{L}} N_{\mathrm{L}}-\mathcal{H}_{\mathrm{L}}^{*}\right)
$$

one has that

One can write $g_{\mathrm{L}}$ as

$$
\mathcal{H}_{\mathrm{L}}\left(N_{\mathrm{L}}\right)=\frac{1}{2 L} N_{\mathrm{L}}^{2}
$$

$$
g_{\mathrm{L}}=g_{\mathrm{L} 11} \mathrm{~d} N_{\mathrm{L}} \otimes \mathrm{d} N_{\mathrm{L}}, \quad g_{\mathrm{L} 11}:=\frac{\mathrm{d}^{2} \mathcal{H}_{\mathrm{L}}}{\mathrm{d} N_{\mathrm{L}}^{2}}=\frac{1}{L}>0 .
$$


It follows from

$$
g_{\mathrm{L} 11} g_{\mathrm{L} 11}^{-1}=1, \quad N_{\mathrm{L}}=\frac{\mathrm{d} \mathcal{H}_{\mathrm{L}}^{*}}{\mathrm{~d} I_{\mathrm{L}}}, \quad I_{\mathrm{L}}=\frac{\mathrm{d} \mathcal{H}_{\mathrm{L}}}{\mathrm{d} N_{\mathrm{L}}}, \quad g_{\mathrm{L} 11}^{-1}=\frac{\mathrm{d}^{2} \mathcal{H}_{\mathrm{L}}^{*}}{\mathrm{~d} I_{\mathrm{L}}^{2}}=\frac{\mathrm{d} N_{\mathrm{L}}}{\mathrm{d} I_{\mathrm{L}}}, \quad g_{\mathrm{L} 11}=\frac{\mathrm{d}^{2} \mathcal{H}_{\mathrm{L}}}{\mathrm{d} N_{\mathrm{L}}^{2}}=\frac{\mathrm{d} I_{\mathrm{L}}}{\mathrm{d} N_{\mathrm{L}}},
$$

that

$$
g_{\mathrm{L}}\left(\frac{\partial}{\partial N_{\mathrm{L}}}, \frac{\partial}{\partial I_{\mathrm{L}}}\right)=1
$$

Therefore, $N_{\mathrm{L}}$ and $I_{\mathrm{L}}$ are mutually dual with respect to $g_{\mathrm{L}}$ ( see Definition 2.17).

The pair $\left(\mathbb{R}, g_{\mathrm{L}}\right)$ is a Riemannian manifold. Let $\nabla_{\mathrm{L}}^{*}$ be the connection such that $g_{\mathrm{L}}=\nabla_{\mathrm{L}}^{*} \mathrm{~d} \mathcal{H}_{\mathrm{L}}^{*}$. Then, $I_{\mathrm{L}}$ is the $\nabla_{\mathrm{L}}^{*}$-affine coordinate, and there exists the unique dual connection $\nabla_{\mathrm{L}}$ due to an analogue of Lemma2.1. Applying Lemma2.2 with $N_{\mathrm{L}}$ and $I_{\mathrm{L}}$ being mutually dual with respect to $g_{\mathrm{L}}$, one has that $\left(\mathbb{R}, g_{\mathrm{L}}, \nabla_{\mathrm{L}}, \nabla_{\mathrm{L}}^{*}\right)$ is a 1-dimensional dually flat space ( see Definition 2.18). In addition, (31) can be seen as a dynamical system on a 1-dimensional dually flat space.

In what follows the dynamical system (31) with the condition $N_{\mathrm{L}}=L I_{\mathrm{L}}$ in (32) is identified with a vector field on the embedded Legendre submanifold generated by $\mathcal{H}_{\mathrm{L}}^{*}$ defined in (32).

Proposition 3.4. ( $R L$ circuit model as a dynamical system on a Legendre submanifold) : Let $\left(\mathcal{C}_{\mathrm{L}}, \lambda_{\mathrm{L}}\right)$ be a contact manifold with $\mathcal{C}_{\mathrm{L}}=\mathbb{R}^{3},\left(N_{\mathrm{L}}, I_{\mathrm{L}}, z_{\mathrm{L}}\right)$ coordinates of $\mathcal{C}_{\mathrm{L}}$, and $\lambda_{\mathrm{L}}=\mathrm{d} z_{\mathrm{L}}-I_{\mathrm{L}} \mathrm{d} N_{\mathrm{L}}$. Then, the vector field on $\mathcal{C}_{\mathrm{L}}$ associated with (31) is on the Legendre submanifold generated by $\mathcal{H}_{\mathrm{L}}^{*}$, and its component expressions are

$$
\frac{\mathrm{d}}{\mathrm{d} t} N_{\mathrm{L}}=-R I_{\mathrm{L}}, \quad \frac{\mathrm{d}}{\mathrm{d} t} I_{\mathrm{L}}=-\frac{R}{L} I_{\mathrm{L}}, \quad \frac{\mathrm{d}}{\mathrm{d} t} z_{\mathrm{L}}=-R I_{\mathrm{L}}^{2},
$$

together with the conditions

$$
N_{L}=L I_{\mathrm{L}}, \quad \text { and } \quad z_{\mathrm{L}}=\frac{L}{2} I_{\mathrm{L}}^{2} .
$$

Proof. Let $F_{\mathrm{L}}$ be the following function of $I_{\mathrm{L}}$

$$
F_{\mathrm{L}}\left(I_{\mathrm{L}}\right)=-\frac{R}{L} I_{\mathrm{L}}
$$

so that (31) can be written as

$$
\frac{\mathrm{d}}{\mathrm{d} t} I_{\mathrm{L}}=F_{\mathrm{L}}\left(I_{\mathrm{L}}\right)
$$

Applying Proposition 3.2. one has that

$$
\frac{\mathrm{d}}{\mathrm{d} t} N_{\mathrm{L}}=\frac{\mathrm{d}}{\mathrm{d} t} \frac{\mathrm{d} \mathcal{H}_{\mathrm{L}}^{*}}{\mathrm{~d} I_{\mathrm{L}}}=-R I_{\mathrm{L}}, \quad \frac{\mathrm{d}}{\mathrm{d} t} I_{\mathrm{L}}=F_{\mathrm{L}}\left(I_{\mathrm{L}}\right)=-\frac{R}{L} I_{L}, \quad \frac{\mathrm{d}}{\mathrm{d} t} z_{\mathrm{L}}=I_{\mathrm{L}} F_{\mathrm{L}}\left(I_{\mathrm{L}}\right) \frac{\mathrm{d}^{2} \mathcal{H}_{\mathrm{L}}^{*}}{\mathrm{~d} I_{\mathrm{L}}^{2}}=-R I_{\mathrm{L}}^{2} .
$$

The vector field associated with this dynamical system is on the Legendre submanifold, where the following relations

$$
\begin{aligned}
\Delta_{\mathrm{L}}^{0}\left(N_{\mathrm{L}}, I_{\mathrm{L}}, z_{\mathrm{L}}\right) & :=N_{L} I_{\mathrm{L}}-\mathcal{H}_{\mathrm{L}}^{*}\left(I_{\mathrm{L}}\right)-z_{\mathrm{L}}=N_{L} I_{\mathrm{L}}-\frac{L}{2} I_{\mathrm{L}}^{2}-z_{\mathrm{L}}=0, \\
\Delta_{\mathrm{L}}^{1}\left(N_{\mathrm{L}}, I_{\mathrm{L}}\right) & :=N_{\mathrm{L}}-\frac{\mathrm{d} \mathcal{H}_{\mathrm{L}}^{*}}{\mathrm{~d} I_{\mathrm{L}}}=N_{\mathrm{L}}-L I_{\mathrm{L}}=0,
\end{aligned}
$$

hold. From these relations, one has that $N_{\mathrm{L}}=L I_{\mathrm{L}}$ and $z_{\mathrm{L}}=L I_{\mathrm{L}}^{2} / 2$. In addition, this vector field is written as $\left.X_{h_{\mathrm{L}}}\right|_{h_{\mathrm{L}}=0}$, where $X_{h_{\mathrm{L}}}$ is the contact Hamiltonian vector field associated to $h_{\mathrm{L}}\left(N_{\mathrm{L}}, I_{\mathrm{L}}, z_{\mathrm{L}}\right)=$ $\Delta_{\mathrm{L}}^{1}\left(N_{\mathrm{L}}, I_{\mathrm{L}}\right) F_{\mathrm{L}}\left(I_{\mathrm{L}}\right)+\Gamma_{\mathrm{L}}\left(\Delta_{\mathrm{L}}^{0}\right)$, with $\Gamma_{\mathrm{L}}$ being a function such that $\Gamma_{\mathrm{L}}(0)=0$ and $\Gamma_{\mathrm{L}}\left(\Delta_{\mathrm{L}}^{0}\right) \neq 0$ for $\Delta_{\mathrm{L}}^{0} \neq 0$. The condition $h_{\mathrm{L}}=0$ is equivalent to the conditions, $\Delta_{\mathrm{L}}^{0}=0$ and $\Delta_{\mathrm{L}}^{1}=0$.

Remark 3.10. The discussions above on the RL circuit model can be extended to those on a class where the magnetic energy

$$
\mathcal{H}_{\mathrm{L}}\left(N_{\mathrm{L}}\right)=\int N_{\mathrm{L}}\left(I_{\mathrm{L}}\right) \mathrm{d} I_{\mathrm{L}},
$$

is a strictly convex function of $N_{\mathrm{L}}$ with $N_{\mathrm{L}}=N_{\mathrm{L}}\left(I_{\mathrm{L}}\right)$ being not necessary to be $N_{\mathrm{L}}=L I_{\mathrm{L}}$. 
Third, a circuit model consisting of a resistor, inductor, and capacitor is focused.

Definition 3.3. ( $R L C$ circuit model) : Let $C$ be a constant capacitor with $C>0, L$ a constant inductor with $L>0, R$ a constant resistor with $R>0, V \in \mathbb{R}$ the capacitor voltage, $I \in \mathbb{R}$ the current, $Q \in \mathbb{R}$ the amount of charge in the capacitor, $N \in \mathbb{R}$ magnetic flux due to $I, \mathcal{H}(Q, N) \in \mathbb{R}$ the total energy stored in the capacitor and the inductor, and $t \in \mathbb{R}$ time. The $R L C$ circuit model is defined as the following system

together with

$$
C \frac{\mathrm{d}}{\mathrm{d} t} V=I, \quad L \frac{\mathrm{d}}{\mathrm{d} t} I=-V-R I,
$$

$$
\mathcal{H}(Q, N):=\frac{Q^{2}}{2 C}+\frac{N^{2}}{2 L}, \quad \text { and } \quad Q=C V, \quad N=L I
$$

Remark 3.11. The dynamical system (34) with (35) can be derived from the energy balance equation.

Theorem 3.8. ( RLC circuit model on a dually flat space) : The system (34) can be seen as a dynamical system on a dually flat space. In particular, the coordinate systems $\{Q, N\}$ and $\{V, I\}$ can be seen as being mutually dual with respect to a metric tensor field.

Proof. Since $\mathcal{H}$ is a strictly convex function on $\mathbb{R}^{2}$ whose coordinates are $Q$ and $N$, one can identify $\left\{\partial^{2} \mathcal{H} / \partial x^{a} \partial x^{b}\right\}$ as the components of a Riemannian metric tensor field $g$, where $x^{1}=Q$ and $x^{2}=N$. These are summarized as

$$
g:=g_{11} \mathrm{~d} Q \otimes \mathrm{d} Q+g_{22} \mathrm{~d} N \otimes \mathrm{d} N, \quad g_{11}:=\frac{\partial^{2} \mathcal{H}}{\partial Q^{2}}=\frac{1}{C}>0, \quad g_{22}:=\frac{\partial^{2} \mathcal{H}}{\partial N^{2}}=\frac{1}{L}>0 .
$$

Defining the total Legendre transform of $\mathcal{H}$ with respect to $\{Q, N\}$ as

$$
\mathcal{H}^{*}(V, I):=\sup _{Q, N}(Q V+N I-\mathcal{H})
$$

one has that

$$
\mathcal{H}^{*}(V, I)=\frac{C}{2} V^{2}+\frac{L}{2} I^{2}
$$

One can write $g$ as

$$
g=g_{11}^{-1} \mathrm{~d} V \otimes \mathrm{d} V+g_{22}^{-1} \mathrm{~d} I \otimes \mathrm{d} I, \quad g_{11}^{-1}:=\frac{\partial^{2} \mathcal{H}^{*}}{\partial V^{2}}=C>0, \quad g_{22}^{-1}:=\frac{\partial^{2} \mathcal{H}^{*}}{\partial I^{2}}=L>0 .
$$

It follows from

$$
g_{11} g_{11}^{-1}=1, \quad g_{22} g_{22}^{-1}=1, \quad V=\frac{\partial \mathcal{H}}{\partial Q}, \quad I=\frac{\partial \mathcal{H}}{\partial N}
$$

and

$$
g_{11}=\frac{\partial^{2} \mathcal{H}}{\partial Q^{2}}=\frac{\partial V}{\partial Q}, \quad g_{11}^{-1}=\frac{\partial^{2} \mathcal{H}^{*}}{\partial V^{2}}=\frac{\partial Q}{\partial V}, \quad g_{22}=\frac{\partial^{2} \mathcal{H}}{\partial N^{2}}=\frac{\partial V}{\partial N}, \quad g_{22}^{-1}=\frac{\partial^{2} \mathcal{H}^{*}}{\partial I^{2}}=\frac{\partial N}{\partial I}
$$

that

$$
g\left(\frac{\partial}{\partial Q}, \frac{\partial}{\partial V}\right)=g\left(\frac{\partial}{\partial N}, \frac{\partial}{\partial I}\right)=1, \quad g\left(\frac{\partial}{\partial Q}, \frac{\partial}{\partial I}\right)=g\left(\frac{\partial}{\partial N}, \frac{\partial}{\partial V}\right)=0 .
$$

Therefore, $\{Q, N\}$ and $\{V, I\}$ are mutually dual with respect to $g$ (see Definition2.17).

The pair $\left(\mathbb{R}^{2}, g\right)$ is a Riemannian manifold. Let $\nabla$ be the connection such that $g=\nabla \mathrm{d} \mathcal{H}$. Then, $\{Q, N\}$ is a set of $\nabla$-affine coordinates, and there exists the unique dual connection $\nabla^{*}$ due to an analogue of Lemma2.1. Applying Lemma2.2 with $\{Q, N\}$ and $\{V, I\}$ being mutually dual with respect to $g$, one has that $\left(\mathbb{R}^{2}, g, \nabla, \nabla^{*}\right)$ is a 2-dimensional dually flat space ( see Definition 2.18). In addition, (34) can be seen as a dynamical system on a 2-dimensional dually flat space.

In what follows the dynamical system (34) with the conditions $Q=C V$ and $N=L I$ in (35) is identified with a vector field on the embedded Legendre submanifold generated by $\mathcal{H}$ in (35). 
Proposition 3.5. ( $R L C$ circuit model as a dynamical system on a Legendre submanifold) : Let $\left(\mathcal{C}_{0}, \lambda_{0}\right)$ be a contact manifold with $\mathcal{C}_{0}=\mathbb{R}^{5},(Q, N, V, I, z)$ coordinates of $\mathcal{C}_{0}$, and $\lambda_{0}=\mathrm{d} z-V \mathrm{~d} Q-I \mathrm{~d} N$. The vector field on $\mathcal{C}_{0}$ associated with (34) is expressed as

$$
\frac{\mathrm{d} Q}{\mathrm{~d} t}=I, \quad \frac{\mathrm{d} N}{\mathrm{~d} t}=-V-R I, \quad \frac{\mathrm{d} V}{\mathrm{~d} t}=\frac{I}{C}, \quad \frac{\mathrm{d} I}{\mathrm{~d} t}=-\frac{V}{L}-\frac{R}{L} I, \quad \frac{\mathrm{d} z}{\mathrm{~d} t}=-R I^{2}
$$

together with the conditions

$$
Q=C V, \quad N=L I, \quad \text { and } \quad z=\frac{C V^{2}}{2}+\frac{L I^{2}}{2} .
$$

Proof. Let $\left\{F_{1}, F_{2}\right\}$ be the following functions of $V$ and $I$

$$
F_{1}(V, I)=\frac{1}{C} I, \quad F_{2}(V, I)=-\frac{V}{L}-\frac{R}{L} I,
$$

so that (34) can be written as

$$
\frac{\mathrm{d}}{\mathrm{d} t} V=F_{1}(V, I), \quad \text { and } \quad \frac{\mathrm{d}}{\mathrm{d} t} I=F_{2}(V, I) .
$$

Applying Proposition 3.2, one has that

$$
\frac{\mathrm{d} Q}{\mathrm{~d} t}=\frac{\mathrm{d}}{\mathrm{d} t} \frac{\partial \mathcal{H}^{*}}{\partial V}=I, \quad \frac{\mathrm{d} N}{\mathrm{~d} t}=\frac{\mathrm{d}}{\mathrm{d} t} \frac{\partial \mathcal{H}^{*}}{\partial I}=-V-R I,
$$

and

$$
\frac{\mathrm{d} V}{\mathrm{~d} t}=F_{1}=\frac{I}{C}, \quad \frac{\mathrm{d} I}{\mathrm{~d} t}=F_{2}=-\frac{V}{L}-\frac{R}{L} I, \quad \frac{\mathrm{d} z}{\mathrm{~d} t}=V F_{1} \frac{\partial^{2} \mathcal{H}^{*}}{\partial V^{2}}+I F_{2} \frac{\partial^{2} \mathcal{H}^{*}}{\partial I^{2}}=-R I^{2} .
$$

The vector field associated with this dynamical system is on the Legendre submanifold, where the following relations

$$
\begin{aligned}
\Delta^{0} & :=Q V+N I-\mathcal{H}^{*}-z=Q V+N I-\left(\frac{C}{2} V^{2}+\frac{L}{2} I^{2}\right)-z=0, \\
\Delta^{1} & :=Q-\frac{\partial \mathcal{H}^{*}}{\partial V}=Q-C V=0, \quad \Delta^{2}:=N-\frac{\partial \mathcal{H}^{*}}{\partial I}=N-L I=0,
\end{aligned}
$$

hold. From these relations, one has that $Q=C V, N=L I$ and $z=\left(C V^{2}+L I^{2}\right) / 2$. In addition, this vector field is written as $\left.X_{h}\right|_{h=0}$, where $X_{h}$ is the contact Hamiltonian vector field associated to $h=\Delta^{1} F_{1}+\Delta^{2} F_{2}+\Gamma\left(\Delta^{0}\right)$, with $\Gamma$ being a function such that $\Gamma(0)=0$ and $\Gamma\left(\Delta^{0}\right) \neq 0$ for $\Delta^{0} \neq 0$. The condition $h=0$ is equivalent to the conditions, $\Delta^{0}=0$ and $\Delta^{1}=\Delta^{2}=0$.

Remark 3.12. The discussions above on the RLC circuit model can be extended to those on a class where the energy

$$
\mathcal{H}(Q, N)=\int Q(V) \mathrm{d} V+\int N(I) \mathrm{d} I
$$

is a strictly convex function of $\{Q, N\}$ with $Q=Q(V)$ and $N=N(I)$ being not necessary to be $Q=C V$ and $N=L I$, respectively.

In Ref. 23], $\mathcal{H}_{\mathrm{C}}^{*}, \mathcal{H}_{\mathrm{L}}^{*}$ and $\mathcal{H}^{*}$ have been referred to as co-energy functions.

\section{Vector fields on contact manifolds lifted from Legendre sub- manifolds}

In this section, a way to give lifts of vector fields on Legendre submanifolds to contact manifolds, basic properties of such lifted vectors fields, and stability of some classes of lifted vector fields are discussed.

In this paper we define the lift of flow and that of dynamical system as follows. 
Definition 4.1. ( Lift of a flow, Lift of a dynamical system ) : Let $\mathcal{M}$ and $\mathcal{N}$ be manifolds with $\operatorname{dim} \mathcal{M}<\operatorname{dim} \mathcal{N}, \pi: \mathcal{N} \rightarrow \mathcal{M}$ a projection, $\Phi_{t}^{\mathcal{M}}: \mathcal{M} \rightarrow \mathcal{M}$ and $\Phi_{t}^{\mathcal{N}}: \mathcal{N} \rightarrow \mathcal{N}$ flows of some dynamical systems $(t \in \mathbb{T} \subset \mathbb{R})$. If $\Phi_{t}^{\mathcal{M}} \circ \pi=\pi \circ \Phi_{t}^{\mathcal{N}}$ is satisfied, then $\Phi_{t}^{\mathcal{N}}$ is referred to as a lift of $\Phi_{t}^{\mathcal{M}}$ ( see the diagram below).

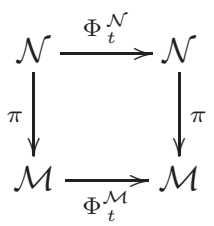

In this paper attention is concentrated on the following two cases.

- $\mathcal{N}=\mathcal{C}, \mathcal{M}=\mathcal{A}_{\psi}$, and the projection $\pi_{\psi}$ is chosen such that $\pi_{\psi}(x, p, z)=x$.

- $\mathcal{N}=\mathcal{C}, \mathcal{M}=\mathcal{A}_{\varphi}$ and the projection $\pi_{\varphi}$ is chosen such that $\pi_{\varphi}(x, p, z)=p$.

\subsection{Lifted vector fields and their basic properties}

In this subsection it is shown how flows on the Legendre submanifold generated by $\psi$ are lifted to $\mathcal{C}$.

Proposition 4.1. (Vector field on $\mathcal{C}$ lifted from the Legendre submanifold generated by $\psi$ ) : Let $\left\{F_{\psi}^{1}, \ldots, F_{\psi}^{n}\right\}$ be a set of functions of $x$ on $\mathcal{A}_{\psi}$, where these functions do not identically vanish. The flow of the vector field associated with the dynamical system defined on $\mathcal{A}_{\psi}$ as

$$
\check{X}_{\psi}^{0}=\dot{x}^{a} \frac{\partial}{\partial x^{a}}, \quad \text { where } \frac{\mathrm{d}}{\mathrm{d} t} x^{a}=F_{\psi}^{a}(x), \quad a \in\{1, \ldots, n\}
$$

is lifted to $\mathcal{C}$ as

$$
X_{h, \psi}=\dot{x}^{a} \frac{\partial}{\partial x^{a}}+\dot{p}_{a} \frac{\partial}{\partial p_{a}}+\dot{z} \frac{\partial}{\partial z},
$$

where $\left\{\dot{x}^{1}, \ldots, \dot{x}^{n}\right\},\left\{\dot{p}_{1}, \ldots, \dot{p}_{n}\right\}, \dot{z}$ satisfy

$$
\frac{\mathrm{d}}{\mathrm{d} t} x^{a}=F_{\psi}^{a}(x), \quad \frac{\mathrm{d}}{\mathrm{d} t} \Delta_{a}=-\frac{\partial F_{\psi}^{b}}{\partial x^{a}} \Delta_{b}-\frac{\mathrm{d} \Gamma_{\psi}}{\mathrm{d} \Delta_{0}} \Delta_{a}, \quad \frac{\mathrm{d}}{\mathrm{d} t} \Delta_{0}=-\Gamma_{\psi}\left(\Delta_{0}\right),
$$

with $\Gamma_{\psi}$ being a function of $\Delta_{0}$. This lifted flow $X_{h, \psi}$, (38), is the contact Hamiltonian vector field associated to the contact Hamiltonian

$$
h_{\psi}(x, p, z)=\Delta_{a}(x, p) F_{\psi}^{a}(x)+\Gamma_{\psi}\left(\Delta_{0}(x, z)\right) .
$$

In addition, if $\Gamma_{\psi}$ is such that $\Gamma_{\psi}(0)=0$ and $\Gamma_{\psi}\left(\Delta_{0}\right) \neq 0$ for $\Delta_{0} \neq 0$, then $X_{\psi}^{0}:=\left.X_{h, \psi}\right|_{\Delta_{0}=\cdots=\Delta_{n}=0}$ is tangent to $\mathcal{A}_{\psi}^{\mathcal{C}}$ ( see the diagrams below)
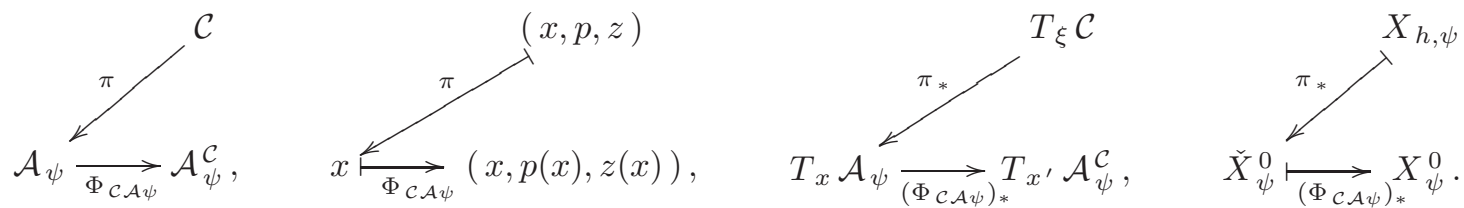

Proof. Substituting the contact Hamiltonian $h_{\psi}$ into (13), one has that

$$
\frac{\mathrm{d} x^{a}}{\mathrm{~d} t}=F_{\psi}^{a}, \quad \frac{\mathrm{d} p_{a}}{\mathrm{~d} t}=\frac{\mathrm{d}}{\mathrm{d} t}\left(\frac{\partial \psi}{\partial x^{a}}\right)+\frac{\partial F_{\psi}^{b}}{\partial x^{a}} \Delta_{b}+\frac{\mathrm{d} \Gamma_{\psi}}{\mathrm{d} \Delta_{0}} \Delta_{a}, \quad \frac{\mathrm{d} z}{\mathrm{~d} t}=\frac{\mathrm{d} \psi}{\mathrm{d} t}+\Gamma_{\psi}\left(\Delta_{0}\right),
$$

from which one has (38). Next, consider systems where $\Gamma_{\psi}(0)=0$ and $\Gamma_{\psi}\left(\Delta_{0}\right) \neq 0$ for $\Delta_{0} \neq 0$ hold. It follows that

$$
\left.X_{h, \psi}\right|_{\Delta_{0}=\cdots=\Delta_{n}=0}=\left.X_{h, \psi}\right|_{h_{\psi}=0}=\left.X_{h, \psi}\right|_{\mathcal{A}_{\psi}^{\mathcal{c}}}
$$


Remark 4.1. The functions $\left\{F_{\psi}^{1}, \ldots, F_{\psi}^{n}\right\}$ need not depend on $\psi$.

There is a counterpart of this Proposition.

Proposition 4.2. (Vector field on $\mathcal{C}$ lifted from the Legendre submanifold generated by $-\varphi$ ) : Let $\left\{F_{1}^{\varphi}, \ldots, F_{n}^{\varphi}\right\}$ be a set of functions of $p$ on $\mathcal{A}_{\varphi}^{\mathcal{C}}$, where these functions do not identically vanish. The flow of the vector field associated with the dynamical system defined on $\mathcal{A}_{\varphi}$ as

$$
X_{\varphi}^{0}=\dot{p}_{a} \frac{\partial}{\partial p_{a}}, \quad \text { where } \frac{\mathrm{d}}{\mathrm{d} t} p_{a}=F_{a}^{\varphi}(p), \quad a \in\{1, \ldots, n\}
$$

is lifted to $\mathcal{C}$ as

$$
X_{h, \varphi}=\dot{x}^{a} \frac{\partial}{\partial x^{a}}+\dot{p}_{a} \frac{\partial}{\partial p_{a}}+\dot{z} \frac{\partial}{\partial z},
$$

where $\left\{\dot{x}^{1}, \ldots, \dot{x}^{n}\right\},\left\{\dot{p}_{1}, \ldots, \dot{p}_{n}\right\}, \dot{z}$ satisfy

$$
\frac{\mathrm{d}}{\mathrm{d} t} \Delta^{a}=-\frac{\partial F_{b}^{\varphi}}{\partial p_{a}} \Delta^{b}-\frac{\mathrm{d} \Gamma^{\varphi}}{\mathrm{d} \Delta^{0}} \Delta^{a}, \quad \frac{\mathrm{d}}{\mathrm{d} t} p_{a}=F_{a}^{\varphi}(p), \quad \frac{\mathrm{d}}{\mathrm{d} t} \Delta^{0}=-\Gamma^{\varphi}\left(\Delta^{0}\right),
$$

with $\Gamma^{\varphi}$ being a function of $\Delta^{0}$. This lifted flow $\left.X_{h, \varphi}, \sqrt{40}\right)$, is the the contact Hamiltonian vector field associated to the contact Hamiltonian

$$
h^{\varphi}(x, p, z)=\Delta^{a}(x, p) F_{a}^{\varphi}(p)+\Gamma^{\varphi}\left(\Delta^{0}(x, p, z)\right) .
$$

In addition, if $\Gamma^{\varphi}$ is such that $\Gamma^{\varphi}(0)=0$ and $\Gamma^{\varphi}\left(\Delta^{0}\right) \neq 0$ for $\Delta^{0} \neq 0$, then $X_{\varphi}^{0}:=\left.X_{h, \varphi}\right|_{\Delta^{0}=\cdots=\Delta^{n}=0}$ is tangent to $\mathcal{A}_{\varphi}^{\mathcal{C}}$ ( see the diagrams below)
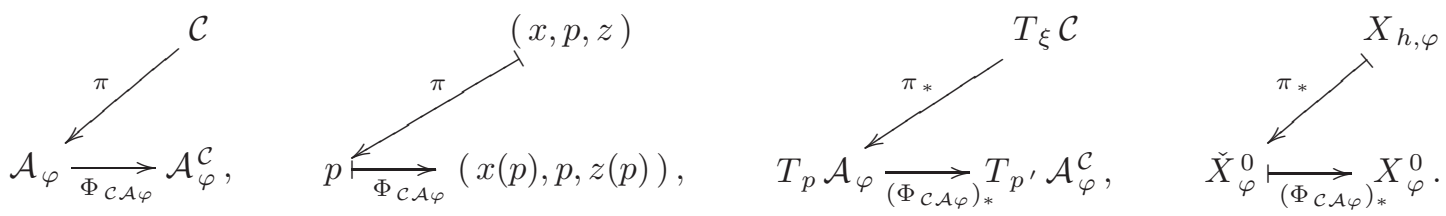

Proof. A way to prove this is analogous to that of Proposition 4.1.

Remark 4.2. The functions $\left\{F_{1}^{\varphi}, \ldots, F_{n}^{\varphi}\right\}$ need not depend on $\varphi$.

The following is a property of the contact Hamiltonian that describes lifted vector fields.

Proposition 4.3. (Differentiation of $h_{\psi}$ with respect to $t$ ) : Consider the system stated in Proposition 4.1. Then, it follows that

$$
\frac{\mathrm{d}}{\mathrm{d} t} h_{\psi}=-\frac{\mathrm{d} \Gamma_{\psi}}{\mathrm{d} \Delta_{0}} h_{\psi}
$$

In the case where $\Gamma_{\psi}\left(\Delta_{0}\right)=\gamma_{0} \Delta_{0}$ with $\gamma_{0}$ being a non-zero constant, it follows that $h_{\psi}(t)=h_{\psi}(0) \exp \left(-\gamma_{0} t\right)$.

Proof. It follows from (38) that

$$
\frac{\mathrm{d}}{\mathrm{d} t} h_{\psi}=\dot{\Delta}_{a} F_{\psi}^{a}+\Delta_{a} \dot{F}_{\psi}^{a}+\frac{\mathrm{d} \Gamma_{\psi}}{\mathrm{d} \Delta_{0}} \dot{\Delta}_{0}=-\frac{\mathrm{d} \Gamma_{\psi}}{\mathrm{d} \Delta_{0}} h_{\psi}
$$

In the case where $\Gamma_{\psi}\left(\Delta_{0}\right)=\gamma_{0} \Delta_{0}$, the solution to this differential equation is $h_{\psi}(t)=h_{\psi}(0) \exp \left(-\gamma_{0} t\right)$.

Remark 4.3. For systems with $\Gamma_{\psi}\left(\Delta_{0}\right)=\gamma_{0} \Delta_{0}$, the subspaces $\left\{(x, p, z) \mid h_{\psi}>0\right\}$ and $\left\{(x, p, z) \mid h_{\psi}<\right.$ $0\} \subset \mathcal{C}$ are invariant ones.

Remark 4.4. Choose a system where $F_{\psi}^{a} \equiv 0,(a \in\{1, \ldots, n\}), \mathrm{d} \Gamma_{\psi} / \mathrm{d} \Delta_{0}>0$, and $h_{\psi}(x(0), p(0), z(0)) \geq$ 0 with the equality holds when $\Delta_{0}=0$. Then, the contact Hamiltonian $h_{\psi}$ is a Lyapunov function ( see Ref. [6] ).

There is a counter part of this Proposition. 
Proposition 4.4. (Differentiation of $h_{\varphi}$ with respect to $t$ ) : Consider the system stated in Proposition 4.2. Then, it follows that

$$
\frac{\mathrm{d}}{\mathrm{d} t} h_{\varphi}=-\frac{\mathrm{d} \Gamma^{\varphi}}{\mathrm{d} \Delta^{0}} h_{\varphi}
$$

In the case where $\Gamma^{\varphi}\left(\Delta^{0}\right)=\gamma^{0} \Delta^{0}$ with $\gamma^{0}$ being a non-zero constant, it follows that $h_{\varphi}(t)=h_{\varphi}(0) \exp \left(-\gamma^{0} t\right)$.

Proof. A way to prove this is analogous to that of Proposition 4.3 .

Remark 4.5. For systems with $\Gamma^{\varphi}\left(\Delta^{0}\right)=\gamma^{0} \Delta^{0}$, the subspaces $\left\{(x, p, z) \mid h_{\varphi}>0\right\} \subset \mathcal{C}$ and $\left\{(x, p, z) \mid h_{\varphi}<\right.$ $0\} \subset \mathcal{C}$ are invariant ones.

Remark 4.6. Choose a system where $F_{a}^{\varphi} \equiv 0,(a \in\{1, \ldots, n\}), \mathrm{d} \Gamma^{\varphi} / \mathrm{d} \Delta^{0}>0$, and $h_{\varphi} \geq 0$ with the equality holds when $\Delta^{0}=0$. Then, the contact Hamiltonian $h_{\varphi}$ is a Lyapunov function ( see Ref. [6] ).

As shown below, the phase compressibility for a class of the lifted vector fields with $\Omega_{\lambda}$ does not depend on $\left\{F_{\psi}^{1}, \ldots, F_{\psi}^{n}\right\}$.

Lemma 4.1. ( Phase compressibility for $X_{h, \psi}$ with $\Omega_{\lambda}$ ) : Consider the system stated in Proposition 4.1. Choose $\Gamma_{\psi}\left(\Delta_{0}\right)=\gamma_{0} \Delta_{0}$ with $\gamma_{0}$ being a constant. Then, the phase compressibility defined in (1) is calculated as

$$
\kappa_{\Omega_{\lambda}}\left(X_{h, \psi}\right)=-(n+1) \gamma_{0}
$$

where $\Omega_{\lambda}$ has been defined in (2).

Proof. With $\mathcal{L}_{X_{h, \psi}} \lambda=\left(\mathcal{R} h_{\psi}\right) \lambda=-\gamma_{0} \lambda$ and $\mathcal{L}_{X_{h, \psi}} \mathrm{d} \lambda=\mathrm{d} \mathcal{L}_{X_{h, \psi}} \lambda=-\gamma_{0} \mathrm{~d} \lambda$, one has that

$$
\mathcal{L}_{X_{h, \psi}} \Omega_{\lambda}=-(n+1) \gamma_{0} \Omega_{\lambda}
$$

Comparing (41) with (11), one completes the proof.

There is a counterpart of this Lemma.

Lemma 4.2. ( Phase compressibility for $X_{h, \varphi}$ with $\Omega_{\lambda}$ ) : Consider the system stated in Proposition 4.2. Choose $\Gamma^{\varphi}\left(\Delta^{0}\right)=\gamma^{0} \Delta^{0}$ with $\gamma^{0}$ being a constant. Then, the phase compressibility defined in (1) is calculated as

$$
\kappa_{\Omega_{\lambda}}\left(X_{h, \varphi}\right)=-(n+1) \gamma^{0}
$$

where $\Omega_{\lambda}$ has been defined in (2).

Proof. A way to prove this is analogous to that of Lemma 4.1

The following theorem and its counterpart are keys to deal with statistical behaviour of the lifted vector fields.

Theorem 4.1. ( Invariant measure for $X_{h, \psi}$ with $\Omega_{\lambda}$ ) : Consider the system stated in Proposition 4.1 with $h_{\psi}(x(0), p(0), z(0))>0$. Choose $\Gamma_{\psi}\left(\Delta_{0}\right)=\gamma_{0} \Delta_{0}$ with $\gamma_{0}$ being a non-zero constant. An invariant measure in the sense of Definition 2.3 is obtained as

$$
\Omega_{h, \psi}^{\text {Inv }}=f_{h, \psi}^{\operatorname{Inv}} \Omega_{\lambda}, \quad \text { where } \quad f_{h, \psi}^{\text {Inv }}=\frac{1}{\mathcal{Z}_{h, \psi}} h_{\psi}^{-(n+1)},
$$

for the class of the form $f_{h, \psi}^{\operatorname{Inv}}=f_{h, \psi}^{\operatorname{Inv}}\left(h_{\psi}\right)$ with $\mathcal{Z}_{h, \psi}$ being a non-zero constant.

Proof. A way to prove this is to verify that $\mathcal{L}_{X_{h, \psi}} \Omega_{h, \psi}^{\text {Inv }}=0$. First, one has that

$$
\begin{aligned}
\mathcal{L}_{X_{h, \psi}} f_{h, \psi}^{\text {Inv }} & =X_{h, \psi} f_{h, \psi}^{\text {Inv }}=\frac{\mathrm{d} f_{h, \psi}^{\text {Inv }}}{\mathrm{d} h_{\psi}}\left(X_{h, \psi} h_{\psi}\right)=\frac{\mathrm{d} f_{h, \psi}^{\text {Inv }}}{\mathrm{d} h_{\psi}}\left(\mathcal{R} h_{\psi}\right) h_{\psi} \\
& =\frac{\mathrm{d} f_{h, \psi}^{\text {Inv }}}{\mathrm{d} h_{\psi}} \frac{\partial h_{\psi}}{\partial z} h_{\psi}=-\frac{\mathrm{d} f_{h, \psi}^{\text {Inv }}}{\mathrm{d} h_{\psi}} \frac{\mathrm{d} \Gamma_{\psi}}{\mathrm{d} \Delta_{0}} h_{\psi}=-\gamma_{0} \frac{\mathrm{d} f_{h, \psi}^{\text {Inv }}}{\mathrm{d} h_{\psi}} h_{\psi},
\end{aligned}
$$


where (5), (12), $f_{h, \psi}^{\text {Inv }}=f_{h, \psi}^{\text {Inv }}\left(h_{\psi}\right)$, and $\Gamma_{\psi}\left(\Delta_{0}\right)=\gamma_{0} \Delta_{0}$ have been used. Second, substituting this expression and (41), one has that

$$
\begin{aligned}
\mathcal{L}_{X_{h, \psi}} \Omega_{h, \psi}^{\text {Inv }} & =\mathcal{L}_{X_{h, \psi}}\left(f_{h, \psi}^{\text {Inv }} \Omega_{\lambda}\right)=\left(\mathcal{L}_{X_{h, \psi}} f_{h, \psi}^{\text {Inv }}\right) \Omega_{\lambda}+f_{h, \psi}^{\text {Inv }}\left(\mathcal{L}_{X_{h, \psi}} \Omega_{\lambda}\right) \\
& =-\gamma_{0}\left[h_{\psi} \frac{\mathrm{d} f_{h, \psi}^{\text {Inv }}}{\mathrm{d} h_{\psi}}+(n+1) f_{h, \psi}^{\text {Inv }}\right] \Omega_{\lambda} .
\end{aligned}
$$

Finally, one observes that the assumed form of $f_{h, \psi}^{\text {Inv }}$ satisfies the equation

$$
\frac{\mathrm{d} f_{h, \psi}^{\mathrm{Inv}}}{\mathrm{d} h_{\psi}}+\frac{n+1}{h_{\psi}} f_{h, \psi}^{\mathrm{Inv}}=0 .
$$

Thus, one has that $\mathcal{L}_{X_{h, \psi}} \Omega_{h, \psi}^{\operatorname{Inv}}=0$.

Remark 4.7. This invariant measure is the same as that in Ref. 13.

Remark 4.8. Due to Remark4.3, the subset $\left\{(x, p, z) \mid h_{\psi}>0\right\} \subset \mathcal{C}$ is invariant one, one has that $f_{h, \psi}^{\text {Inv }}>0$.

There is a counterpart of this Theorem.

Theorem 4.2. ( Invariant measure for $X_{h, \varphi}$ with $\Omega_{\lambda}$ ) : Consider the system stated in Proposition 4.2 with $h_{\varphi}(x(0), p(0), z(0))>0$. Choose $\Gamma^{\varphi}\left(\Delta^{0}\right)=\gamma^{0} \Delta^{0}$ with $\gamma^{0}$ being a non-zero constant. An invariant measure in the sense of Definition 2.3 is obtained as

$$
\Omega_{h, \varphi}^{\operatorname{Inv}}=f_{h, \varphi}^{\operatorname{Inv}} \Omega_{\lambda}, \quad \text { where } f_{h, \varphi}^{\operatorname{Inv}}=\frac{1}{\mathcal{Z}_{h, \varphi}} h_{\varphi}^{-(n+1)},
$$

for the class of the form $f_{h, \varphi}^{\operatorname{Inv}}=f_{h, \varphi}^{\operatorname{Inv}}\left(h_{\varphi}\right)$ with $\mathcal{Z}_{h, \varphi}$ being a non-zero constant.

Proof. A way to prove this is analogous to that of Theorem 4.1.

Remark 4.9. Due to Remark4.5 the subset $\left\{(x, p, z) \mid h_{\varphi}>0\right\} \subset \mathcal{C}$ is invariant one, one has $f_{h, \varphi}^{\operatorname{Inv}}>0$.

\subsection{Stability theorems}

In this subsection, we show several classes where lifted flows asymptotically approach to the Legendre submanifold.

When $\left\{F_{\psi}^{1}, \ldots, F_{\psi}^{n}\right\}$ and $\Gamma_{\psi}$ in (38) have some properties, it is shown below that flows asymptotically approach to $\mathcal{A}_{\psi}^{\mathcal{C}}$.

Theorem 4.3. ( Stability theorem 1) : Let $\gamma_{0}$ and $\Lambda_{0}$ be non-zero constants. Consider the dynamical system (38) with

$$
\frac{\partial F_{\psi}^{b}}{\partial x^{a}}=\Lambda_{0} \delta_{a}^{b}, \quad \text { and } \quad \Gamma_{\psi}\left(\Delta_{0}\right)=\gamma_{0} \Delta_{0} .
$$

If the conditions

$$
\Lambda_{0}+\gamma_{0}>0, \text { and } \gamma_{0}>0,
$$

are satisfied, then the flow asymptotically approaches to $\mathcal{A}_{\psi}^{\mathcal{C}}$.

Proof. The solutions $\Delta_{0}(t)$ and $\Delta_{a}(t)$ are obtained by solving (38) as

$$
\Delta_{0}(t)=\mathrm{e}^{-\gamma_{0} t} \Delta_{0}(0), \quad \text { and } \quad \Delta_{a}(t)=\mathrm{e}^{-\left(\gamma_{0}+\Lambda_{0}\right) t} \Delta_{a}(0) .
$$

From these equations one has that

$$
\lim _{t \rightarrow \infty} \Delta_{0}(t)=0, \text { and } \lim _{t \rightarrow \infty} \Delta_{a}(t)=0,
$$

or equivalently

$$
\psi(x(\infty))=z(\infty), \quad \text { and } \quad \frac{\partial \psi}{\partial x^{a}}(x(\infty))=p_{a}(\infty),
$$

which are the conditions that the flow asymptotically approaches to $\mathcal{A}_{\psi}^{\mathcal{C}}$. 
Remark 4.10. When $\Lambda_{0}=0$, this dynamics is identical to the relaxation process discussed in Ref. [6].

There is a counterpart of this theorem.

Theorem 4.4. ( Stability theorem 2) : Let $\gamma^{0}$ and $\Lambda^{0}$ be non-zero constants. Consider the dynamical system (40) with

$$
\frac{\partial F_{b}^{\varphi}}{\partial p_{a}}=\Lambda^{0} \delta_{b}^{a}, \quad \text { and } \quad \Gamma^{\varphi}\left(\Delta^{0}\right)=\gamma^{0} \Delta^{0}
$$

If the conditions

$$
\Lambda^{0}+\gamma^{0}>0, \text { and } \gamma^{0}>0
$$

are satisfied, then the flow asymptotically approaches to $\mathcal{A}_{\varphi}^{\mathcal{C}}$.

Proof. A way to prove this is analogous to that of Theorem 4.3 .

In Theorem 4.3. asymptotic behaviour is stated for the case where $\left(\partial F_{\psi}^{a} / \partial x^{b}\right)$ is diagonal and $\left\{x^{1}, \ldots, x^{n}\right\}$ is exponentially increasing or decreasing in $t$. In the following, asymptotic behavior is stated for a class of systems where $\left\{x^{1}(t), \ldots, x^{n}(t)\right\}$ is oscillatory in $t$.

Theorem 4.5. ( Stability theorem 3) : Let $n=2$, and $\gamma_{0}>0, \omega$ constants. Consider the dynamical system (38) with

$$
F_{\psi}^{1}\left(x^{1}, x^{2}\right)=\omega x^{2}, \quad F_{\psi}^{2}\left(x^{1}, x^{2}\right)=-\omega x^{1}, \quad \text { and } \quad \Gamma_{\psi}\left(\Delta_{0}\right)=\gamma_{0} \Delta_{0} .
$$

Then the flow asymptotically approaches to $\mathcal{A}_{\psi}^{\mathcal{C}}$.

Proof. The contact Hamiltonian flow is expressed as

$$
\frac{\mathrm{d} x^{a}}{\mathrm{~d} t}=F_{\psi}^{a} \cdot \quad \frac{\mathrm{d}}{\mathrm{d} t}\left(\begin{array}{c}
\Delta_{1} \\
\Delta_{2}
\end{array}\right)=\left(\begin{array}{cc}
-\gamma_{0} & \omega \\
-\omega & -\gamma_{0}
\end{array}\right)\left(\begin{array}{c}
\Delta_{1} \\
\Delta_{2}
\end{array}\right), \quad \frac{\mathrm{d}}{\mathrm{d} t} \Delta_{0}=-\gamma_{0} \Delta_{0} .
$$

The solutions for $\Delta_{0}, \Delta_{1}$ and $\Delta_{2}$ are given by

$$
\begin{aligned}
& \Delta_{0}(t)=\mathrm{e}^{-\gamma_{0} t} \Delta_{0}(0), \\
& \Delta_{1}(t)=\mathrm{e}^{-\gamma_{0} t}\left[\Delta_{1}(0) \cos (\omega t)+\Delta_{2}(0) \sin (\omega t)\right], \\
& \Delta_{2}(t)=\mathrm{e}^{-\gamma_{0} t}\left[-\Delta_{1}(0) \sin (\omega t)+\Delta_{2}(0) \cos (\omega t)\right] .
\end{aligned}
$$

From these equations one has that

$$
\lim _{t \rightarrow \infty} \Delta_{0}(t)=\lim _{t \rightarrow \infty} \Delta_{1}(t)=\lim _{t \rightarrow \infty} \Delta_{2}(t)=0,
$$

or, equivalently

$$
\psi(x(\infty))=z(\infty), \quad \text { and } \quad \frac{\partial \psi}{\partial x^{a}}(x(\infty))=p_{a}(\infty), \quad a \in\{1,2\}
$$

which are the conditions that the flow asymptotically approaches to $\mathcal{A}_{\psi}^{\mathcal{C}}$.

There is a counterpart of this theorem.

Theorem 4.6. (Stability theorem 4) : Let $n=2$, and $\gamma^{0}>0, \omega$ constants. Consider the dynamical system (40) with

$$
F_{1}^{\varphi}\left(p_{1}, p_{2}\right)=\omega p_{2}, \quad F_{2}^{\varphi}\left(p_{1}, p_{2}\right)=-\omega p_{1}, \quad \text { and } \quad \Gamma^{\varphi}\left(\Delta^{0}\right)=\gamma^{0} \Delta^{0}
$$

Then the flow asymptotically approaches to $\mathcal{A}_{\varphi}^{\mathcal{C}}$.

Proof. A way to prove this is analogous to that of Theorem4.5.

So far the given theorems above do not involve any fixed point for $x$. However, the following involves a fixed point for $x$. 
Theorem 4.7. (Stability theorem 5): Let $\gamma_{0}>0$ be a constant, $\left(L^{a b}\right)$ a constant symmetric positive definite matrix, and $\mathcal{U}_{\psi}$ a positive function of $x$ only. Consider the dynamical system (38) with

$$
F_{\psi}^{a}(x)=-L^{a j} \frac{\partial \mathcal{U}_{\psi}}{\partial x^{j}}, \quad \text { and } \quad \Gamma_{\psi}\left(\Delta_{0}\right)=\gamma_{0} \Delta_{0}
$$

If the condition

$$
\gamma_{0} L^{a b}-L^{a k} \frac{\partial^{2} \mathcal{U}_{\psi}}{\partial x^{k} \partial x^{j}} L^{j b} \quad \text { are components of a positive definite matrix for all } x
$$

is satisfied, then the flow asymptotically approaches to a fixed point in $\mathcal{A}_{\psi}^{\mathcal{C}}$. Here the fixed point $(\bar{x}, \bar{p}, \bar{z}) \in$ $\mathcal{A}_{\psi}^{\mathcal{C}}$ is determined by

$$
L^{a j} \frac{\partial \mathcal{U}_{\psi}}{\partial x^{j}}(\bar{x})=0, \quad \overline{\Delta_{0}}=\overline{\Delta_{1}}=\cdots=\overline{\Delta_{n}}=0 .
$$

Proof. It is shown below that there exists a Lyapunov function. Then applying the stability theorem, one can complete the proof ( see Ref. [24] ). Define the function $V$ on $\mathcal{C}$ as

$$
V(x, p, z)=\mathcal{U}_{\psi}(x)+\frac{1}{2} \Delta_{a}(x, p) L^{a b} \Delta_{b}(x, p)+\frac{1}{2}\left(\Delta_{0}(x, z)\right)^{2} .
$$

Then it follows that $V \geq 0$, and

$$
\dot{V}=-\frac{\partial \mathcal{U}_{\psi}}{\partial x^{b}} L^{b a} \frac{\partial \mathcal{U}_{\psi}}{\partial x^{a}}-\Delta_{b}\left(\gamma_{0} L^{b a}-L^{b k} \frac{\partial^{2} \mathcal{U}_{\psi}}{\partial x^{k} \partial x^{j}} L^{j a}\right) \Delta_{a}-\gamma_{0}\left(\Delta_{0}\right)^{2} .
$$

Thus if the condition is satisfied, then $\dot{V} \leq 0$. On $\left(\bar{x}, \overline{\Delta_{0}}, \overline{\Delta_{1}}, \ldots, \overline{\Delta_{n}}\right)$, the equality holds. Thus, $V$ is a Lyapunov function.

Remark 4.11. The function $\mathcal{U}_{\psi}$ need not depend on $\psi$.

There is a counterpart of this Theorem.

Theorem 4.8. ( Stability theorem 6) : Let $\gamma^{0}>0$ be a constant, $\left(L_{a b}\right)$ components of a constant symmetric positive definite matrix, and $\mathcal{U}^{\varphi}$ a positive function of $p$ only. Consider the dynamical system 40) with

$$
F_{a}^{\varphi}(p)=-L_{a j} \frac{\partial \mathcal{U}^{\varphi}}{\partial p_{j}}, \quad \text { and } \quad \Gamma^{\varphi}\left(\Delta^{0}\right)=\gamma^{0} \Delta^{0} .
$$

If the condition

$$
\gamma^{0} L_{a b}-L_{a k} \frac{\partial^{2} \mathcal{U}^{\varphi}}{\partial p_{k} \partial p_{j}} L_{j b} \quad \text { are components of a positive definite matrix for all } p
$$

is satisfied, then the flow asymptotically approaches to a fixed point in $\mathcal{A}_{\varphi}^{\mathcal{C}}$. Here the fixed point $(\bar{x}, \bar{p}, \bar{z}) \in$ $\mathcal{A}_{\psi}^{\mathcal{C}}$ is determined by

$$
L_{a j} \frac{\partial \mathcal{U}^{\varphi}}{\partial p_{j}}(\bar{p})=0, \quad \overline{\Delta^{0}}=\overline{\Delta^{1}}=\cdots=\overline{\Delta^{n}}=0 .
$$

Proof. A way to prove this is analogous to that of Theorem 4.7

Remark 4.12. The function $\mathcal{U}_{\varphi}$ need not depend on $\varphi$.

\subsection{Application to a spin system under time-dependent controlled magnetic field}

In this subsection it is shown how useful the general theory is in physics by analyzing a simple model. Since contact geometry is used for geometrization of thermodynamics and statistical mechanics, a variety of examples are expected to be found for this purpose. In this paper a nonequilibrium system is focused since it involves naturally time-development physical quantities. We choose as an example a spin system in contact with a time-dependent external magnetic field and time-independent heat bath, since this 
example is simple enough. In the case where the system is in contact with time-independent heat bath and time-independent magnetic field, the equilibrium state and a relaxation process have been studied in Ref. 6]. Thus one can compare the present case and the previous one. Here relaxation process is defined such that every physical quantity approaches to the one at the equilibrium state as time develops, and equilibrium state is defined such that the canonical distribution of microscopic variables is realized. If the equilibrium state is realized for a given system and the corresponding cumulant generating function is analytically obtained, then an expectation value is in principle calculated by differentiating the cumulant generating function with respect to the thermodynamic conjugate variable ( see Definition 4.2 ).

Symbols for physical variables used in this subsection are summarized in Table 1.

\begin{tabular}{|c|l|}
\hline symbol & interpretation \\
\hline$t$ & time \\
$T_{\mathrm{abs}}$ & absolute temperature \\
$k_{\mathrm{B}}$ & Boltzmann constant \\
$H$ & magnetic field whose dimension is an energy \\
$\sigma$ & spin that takes values \pm 1 \\
$\mathcal{F}$ & Helmholtz free energy \\
\hline
\end{tabular}

Table 1: Symbols for physical quantities

In the following the definition of a spin system at the equilibrium state is given.

Definition 4.2. (Equilibrium state of a spin system with an external constant magnetic field in contact with a heat bath ) : Let $\sigma= \pm 1$ be a spin variable, $H$ a spatially homogeneous and time-independent external magnetic field whose physical dimension is an energy, and $\theta:=H /\left(k_{B} T_{\mathrm{abs}}\right)$. Then the canonical distribution function for this system is defined to be

$$
\mathbb{P}_{\theta}^{\operatorname{can}}(\sigma):=\exp (\theta \sigma-\psi(\theta)),
$$

where $\psi(\theta)$ is to normalize $\mathbb{P}_{\theta}^{\text {can }}(\sigma)$ such that $\sum_{\sigma= \pm 1} \mathbb{P}_{\theta}^{\text {can }}(\sigma)=1$ as

$$
\psi(\theta)=\ln \cosh \theta+\ln 2 \text {. }
$$

In addition, the equilibrium value of the magnetization is defined to be

$$
\eta(\theta):=\frac{\partial \psi}{\partial \theta}=\langle\sigma\rangle_{\mathrm{can}}=\tanh \theta
$$

where

$$
\langle\mathcal{O}\rangle_{\text {can }}:=\sum_{\sigma= \pm 1} \mathcal{O}(\sigma) \mathbb{P}_{\theta}^{\text {can }}(\sigma)
$$

for a given function $\mathcal{O}$ of $\sigma$. The macroscopic equilibrium state for this system is defined to be the triplet $(\theta, \eta(\theta), \psi(\theta))$.

The physical interpretation of $\psi$ is the cumulant generating function or the negative dimensionless Helmholtz free energy : $\psi=-\mathcal{F} /\left(k_{\mathrm{B}} T_{\mathrm{abs}}\right)$ ( see Ref. [6] ). Instead of $\psi$, a partition function $Z(\theta)$ is used in physics to normalize a distribution function. The relation between $Z(\theta)$ and $\psi(\theta)$ is given by $\psi(\theta)=\ln Z(\theta)$.

To geometrically describe a relaxation dynamics, where the system is in contact with time-dependent external magnetic field and time-independent heat bath, the following postulates are made.

Postulate 4.1. 1. Coordinates $(x, p, z)$ of a 3 -dimensional contact manifold $(\mathcal{C}, \lambda)$ can be introduced such that $\lambda=\mathrm{d} z-p \mathrm{~d} x$, and that

$$
\left.(x, p, z)\right|_{\mathcal{A}_{\psi}^{\mathcal{c}}}=(\theta, \eta(\theta), \psi(\theta)) .
$$

2. The domain of $\psi$ can be extended such that one can write $\psi(x)$. 
The physical meaning of the Legendre submanifold generated by $\psi$ is the subspace where the thermodynamic relation $\eta(\theta)=\partial \psi / \partial \theta$ holds and the value of the negative dimensionless Helmholtz free energy is the same as that calculated by $\psi, z(\theta)=\psi(\theta)$. Furthermore, when $T_{\text {abs }}$ does not depend of $t$, physical interpretations of canonical coordinates $(x, p, z)$ for the contact manifold are given as follows.

- $x(t)$ can express the time-dependent magnetic field.

At equilibrium, it follows that $\left.x\right|_{\mathcal{A}_{\psi}^{c}}=\theta=H /\left(k_{\mathrm{B}} T_{\text {abs }}\right)$.

- $p(t)$ is the time-dependent magnetization.

At equilibrium, it follows that $\left.p\right|_{\mathcal{A}_{\psi}^{c}}=\langle\sigma\rangle_{\text {can }}=\eta=\partial \psi(\theta) / \partial \theta$.

- $z(t)$ is the time-dependent negative dimensionless Helmholtz free energy.

At equilibrium, it follows that $\left.z\right|_{\mathcal{A}_{\psi}^{c}}=-\mathcal{F}(\theta) /\left(k_{\mathrm{B}} T_{\text {abs }}\right)=\psi(\theta)$.

In the special case where $x$ is constant in time, the contact Hamiltonian vector field associated with $h=\gamma_{0}(\psi(x)-z)$ has been studied in Ref. [6]. The vector field is expressed in this case as

$$
\frac{\mathrm{d} x}{\mathrm{~d} t}=0, \quad \frac{\mathrm{d} p}{\mathrm{~d} t}=\gamma_{0}\left(\frac{\mathrm{d} \psi}{\mathrm{d} x}(x)-p\right), \quad \frac{\mathrm{d} z}{\mathrm{~d} t}=\gamma_{0}(\psi(x)-z),
$$

where $\psi(x)=\ln \cosh (x)+\ln 2$. This vector field has been shown to include a kinetic spin model without spin-coupling, where that model is obtained from a master equation under the detailed balance condition.

The system where $x$ is controlled such that $x$ approaches to a fixed value $\theta$ as time develops can be considered by means of the developed general theory of this paper. As an application of Theorem 4.3 . one has the model which shows a relaxation process in contact with a controlled magnetic field as follows.

Theorem 4.9. (Relaxation dynamics of a spin system in controlled magnetic field ) : Let $(\mathcal{C}, \lambda)$ be a contact manifold with $\mathcal{C}=\mathbb{R}^{3}$ whose coordinates are $(x, p, z)$, and $\lambda=\mathrm{d} z-p \mathrm{~d} x$. In addition, $\Lambda_{0}, \theta$ and $\gamma_{0}$ be positive constants. Consider the system where $T_{\mathrm{abs}}$ is constant in time, the magnetic field divided by $k_{B} T_{\text {abs }}$, denoted $x(t)$, is controlled as

$$
x(t)=(x(0)-\theta) \mathrm{e}^{-\Lambda_{0} t}+\theta,
$$

and the condition $\gamma_{0}>\Lambda_{0}$ is satisfied. Then the contact Hamiltonian vector field associated with (39)

$$
\begin{aligned}
\frac{\mathrm{d}}{\mathrm{d} t} x & =-\Lambda_{0}(x-\theta), \\
\frac{\mathrm{d}}{\mathrm{d} t} p & =-\Lambda_{0}(x-\theta) \frac{\mathrm{d}^{2} \psi}{\mathrm{d} x^{2}}(x)-\Lambda_{0}\left(\frac{\mathrm{d} \psi}{\mathrm{d} x}(x)-p\right)+\gamma_{0}\left(\frac{\mathrm{d} \psi}{\mathrm{d} x}(x)-z\right), \\
\frac{\mathrm{d}}{\mathrm{d} t} z & =-\Lambda_{0}(x-\theta) \frac{\mathrm{d} \psi}{\mathrm{d} x}(x)+\gamma_{0}(\psi(x)-z),
\end{aligned}
$$

with $\psi(x)=\ln \cosh (x)+\ln 2$, or equivalently,

$$
\frac{\mathrm{d}}{\mathrm{d} t} x=-\Lambda_{0}(x-\theta), \quad \frac{\mathrm{d}}{\mathrm{d} t} \Delta_{1}=\Lambda_{0} \Delta_{1}-\gamma_{0} \Delta_{1}, \quad \frac{\mathrm{d}}{\mathrm{d} t} \Delta_{0}=-\gamma_{0} \Delta_{0},
$$

with

$$
\Delta_{0}(x, z)=\psi(x)-z, \quad \Delta_{1}(x, p)=\frac{\mathrm{d} \psi}{\mathrm{d} x}(x)-p,
$$

models a relaxation dynamics under the controlled magnetic field:

$$
x(\infty)=\theta, \quad \psi(x(\infty))=z(\infty), \quad \frac{\partial \psi}{\partial x}(x(\infty))=p(\infty) .
$$

Proof. Applying Theorem 4.3 , one completes the proof.

In the case $\Lambda_{0} \equiv 0$, this system reduces to (42) that includes the kinetic spin model without control. Although it interesting to have the corresponding kinetic model in contact with the controlled magnetic field, where the model is constructed based on a master equation, such a model has not been known. 


\subsection{Application of the general theory to a class of phenomenological equa- tions in nonequilibrium thermodynamics}

The aim of this subsection is to show how Theorem 4.7 is applied to nonequilibrium thermodynamics. In particular, the so-called phenomenological equations are considered.

In nonequilibrium thermodynamics, it has been proposed that time evolution of some macroscopic variables $x=\left\{x^{1}, \ldots, x^{n}\right\} \in \mathbb{R}^{n}$ is described by the following gradient system

$$
\frac{\mathrm{d}}{\mathrm{d} t} x^{a}=-L^{a j} \frac{\partial}{\partial x^{j}} \mathcal{U}(x)=: F^{a}(x),
$$

where $\left\{L^{a b}\right\}$ is identified with a set of the Onsager coefficients, $\mathcal{U}$ a free energy or entropy 25$]$. These systems are referred to as phenomenological equations and often assumed to be valid near the equilibrium state. In this paper, we assume that this class of systems is described at the state where the following thermodynamic relations

$$
p_{a}=\frac{\partial \psi}{\partial x^{a}}(x), \quad a \in\{1, \ldots, n\}
$$

are satisfied, with $p=\left\{p_{1}, \ldots, p_{n}\right\}$ being a set of thermodynamic conjugate variables of $x$, and $\psi$ some potential. Note that under a similar assumption, a similar system has been studied in Ref. [26], where a dynamical system has been derived on the Legendre submanifold generated by a function whose physical interpretation is an energy. In their system it has been shown that the energy is conserved and the entropy increases as time develops. Note also that in general, if a dynamical system is on the Legendre submanifold, then its standard physical interpretation in the contact geometric thermodynamics is that the system is at equilibrium 3 .

In contact geometry under our assumption, and introducing a new variable $z$ such that $z=\psi(x)$, (43) can be seen as a dynamical system on the $n$-dimensional Legendre submanifold generated by $\psi$ of a $(2 n+1)$-dimensional contact manifold. With our framework on how to lift a dynamical system to the contact manifold, such a lifted dynamical system is immediately obtained as (38) with $F^{a}(x)$ given by (43). This lifted dynamical system can be a candidate of a dynamical system that describes some nonequilibrium thermodynamics. The stability of this lifted system is understood as Theorem 4.7.

If the system is restricted to the state where (44) is satisfied, and has some special properties, one has the following.

Proposition 4.5. (Dually flat space for phenomenological equations at equilibrium): Consider the system 43), where all the following conditions are satisfied

- The relation 44) holds.

- The matrix $\left(L_{a b}\right)$ is a constant symmetric positive definite matrix

- The introduced potential is identical to $\psi$ and is of the form

$$
\mathcal{U}(x)=\psi(x)=\frac{1}{2} x^{a} M_{a b} x^{b}
$$

where $\left(M_{a b}\right)$ is the inverse matrix of $\left(L^{a b}\right)$.

Then, this system can be seen as a dynamical system on a dually flat space with the metric tensor field $g=L_{a b} \mathrm{~d} x^{a} \otimes \mathrm{d} x^{b}$, and $x$ and $p$ are mutually dual with respect to $g$. In addition, integral curves in $p$-coordinates trace $\nabla^{*}$-geodesic curves connecting $p(0)$ and $p=0$ where $\nabla^{*}$ is the dual connection of $\nabla$ that is defined such that $\nabla \mathrm{d} \psi=g$.

Proof. It immediately follows from $\nabla \mathrm{d} \psi=g$ that $x$ is a set of $\nabla$-affine coordinates. Introducing $\varphi(p):=\mathfrak{L}[\psi](p)$, which is a strictly convex function, one has

$$
x^{a}=\frac{\partial \varphi}{\partial p_{a}}, \quad \text { and } \quad \mathrm{d} x^{a}\left(\frac{\partial}{\partial p_{b}}\right)=M^{a b},
$$

from which one concludes that $x$ and $p$ are mutually dual with respect to $g$ :

$$
g\left(\frac{\partial}{\partial x^{a}}, \frac{\partial}{\partial p_{b}}\right)=\delta_{a}^{b} .
$$


In addition, the dual connection $\nabla^{*}$ exists due to Lemma 2.1 and the connection coefficients $\left\{\Gamma_{a b}^{\prime c}\right\}$ such that $\nabla_{\partial_{a}}^{\prime} \partial^{b}=\Gamma_{a b}^{\prime} \partial_{c},\left(\partial_{a}:=\partial / \partial x^{a}\right)$ are

$$
\Gamma_{a b}^{\prime c}=g^{c j} \Gamma_{a b j}, \quad \text { with } \quad \Gamma_{a b c}^{\prime}=\frac{\partial^{3} \psi}{\partial x^{a} \partial x^{b} \partial x^{c}}
$$

It follows from Lemma2.2 that $p$ is a set of $\nabla^{*}$-affine coordinates. Therefore, $\left(\mathbb{R}^{n}, g, \nabla, \nabla^{*}\right)$ is a dually flat space, where $\nabla$-affine coordinates are $x$, and $\nabla^{*}$-affine coordinates $p$. In addition, it follows that

$$
\frac{\mathrm{d}}{\mathrm{d} t} p_{a}=\frac{\mathrm{d}}{\mathrm{d} t}\left(\frac{\partial \psi}{\partial x^{a}}\right)=M_{a b} \frac{\mathrm{d} x^{b}}{\mathrm{~d} t}=-M_{a b} L^{b j} \frac{\partial \psi}{\partial x^{j}}=-p_{a}
$$

whose solution for each $a$ is obtained as

$$
p_{a}(t)=p_{a}(0) \mathrm{e}^{-t}
$$

\section{Generating function conserving lifting scheme for contact Hamil- tonian vector fields}

Consider the vector field $X$ on the embedded Legendre submanifold obtained by the push-forward of a vector field $X_{0}$, where the Legendre submanifold is generated by a function $\psi$, and $X=\Phi_{*} X_{0}$ holds with $\Phi$ being an embedding. As mentioned in Remark 3.2 , the value of the generating function $\psi$ is not conserved in the sense that $\mathcal{L}_{X} \psi \not \equiv 0$. If one requires that the value of the generating function is conserved, then another formulation is needed. In this section, such a formulation is shown with a higher dimensional contact manifold. Note that this formulation can be seen as a generalization of the work in Ref. [26].

After fixing mathematical symbols that will be used in Subsections 5.2 5.3, such vector fields on dually flat spaces and their lifts will be investigated.

\subsection{Mathematical symbols}

In this subsection mathematical symbols are fixed as follows. Let $(\widetilde{\mathcal{C}}, \widetilde{\lambda})$ be a $(2 n+3)$-dimensional contact manifold with some fixed $n \in\{1,2, \ldots\}$, and $\left(x, x^{n+1}, p, p_{n+1}, z\right)$ canonical coordinates such that $\widetilde{\lambda}=\mathrm{d} z-p_{a} \mathrm{~d} x^{a}-p_{n+1} \mathrm{~d} x^{n+1}$ with $x=\left\{x^{1}, \ldots, x^{n}\right\}$ and $p=\left\{p_{1}, \ldots, p_{n}\right\}$.

Proposition 5.1. ( Coordinate expression of the Reeb vector field on $\widetilde{\mathcal{C}}$ ) : The coordinate expression of the Reeb vector field on $(\widetilde{\mathcal{C}}, \widetilde{\lambda})$ is given by

$$
\widetilde{\mathcal{R}}=\frac{\partial}{\partial z}
$$

Proof. This is proved by substituting this expression of $\widetilde{\mathcal{R}}$ into (4).

To discuss phase compressibilities of vector fields and invariant measures, the following $(2 n+3)$-form will be used.

Definition 5.1. ( Standard volume-form on a high-dimensional contact manifold) :

$$
\widetilde{\Omega}_{\widetilde{\lambda}}:=\widetilde{\lambda} \wedge \underbrace{\mathrm{d} \widetilde{\lambda} \wedge \cdots \wedge \mathrm{d} \widetilde{\lambda}}_{n+1} .
$$

Define a function on $\widetilde{\mathcal{C}}$

$$
\widetilde{\psi}\left(x, x^{n+1}\right)=\psi(x)+p_{n+1}^{(0)} x^{n+1},
$$


where $p_{n+1}^{(0)}$ is a non-zero constant, and $\psi$ a function. Then, applying Theorem 2.4, one has the $(n+1)$ dimensional Legendre submanifold $\widetilde{\mathcal{A}}_{\widetilde{\psi}}$ generated by $\widetilde{\psi}$, such that

$$
\widetilde{\mathcal{A}}_{\widetilde{\psi}}^{\widetilde{\mathcal{C}}}:=\Phi_{\widetilde{\mathcal{C}} \widetilde{\mathcal{\psi}}} \widetilde{\mathcal{A}}_{\widetilde{\psi}}=\left\{\left(x, x^{n+1}, p, p_{n+1}, z\right) \in \widetilde{\mathcal{C}} \mid p_{j}=\frac{\partial \psi}{\partial x^{j}}, p_{n+1}=p_{n+1}^{(0)}, z=\widetilde{\psi}, j \in\{1, \ldots, n\}\right\},
$$

where $\Phi_{\widetilde{\mathcal{C}} \widetilde{\mathcal{A}}}: \widetilde{\mathcal{A}}_{\widetilde{\psi}} \rightarrow \widetilde{\mathcal{C}}$ is an embedding. The following functions

$$
\widetilde{\Delta}_{0}\left(x, x^{n+1}, z\right):=\widetilde{\psi}\left(x, x^{n+1}\right)-z, \quad \widetilde{\Delta}_{a}\left(x, p, p_{n+1}\right):=\frac{p_{n+1}}{p_{n+1}^{(0)}} \frac{\partial \psi}{\partial x^{a}}(x)-p_{a}
$$

will be used. It follows that

$$
\left.\widetilde{\Delta}_{0}\right|_{\widetilde{\mathcal{A}}_{\tilde{\psi}}^{\tilde{\mathcal{\psi}}}}=0, \quad \text { and }\left.\quad \widetilde{\Delta}_{a}\right|_{\widetilde{\mathcal{A}} \tilde{\tilde{\psi}}}=0, \quad a \in\{1, \ldots, n\} .
$$

In addition, since the matrix components which are differentiation of $\widetilde{\psi}$ with respect to $x$ and $x^{n+1}$ are obtained as

$$
\widetilde{g}_{\mu \nu}=\left(\begin{array}{cc}
\frac{\partial^{2} \psi}{\partial x^{a} \partial x^{b}} & 0 \\
0 & 0
\end{array}\right), \quad a, b \in\{1, \ldots, n\}, \quad \mu, \nu \in\{1, \ldots, n+1\}
$$

the matrix $\left(\widetilde{g}_{\mu \nu}\right)$ provides neither a Riemannian metric tensor field nor a pseudo-Riemannian metric tensor field on $\widetilde{\mathcal{A}}_{\widetilde{\psi}}$.

Similarly one has the counterpart. Define a function on $\widetilde{\mathcal{C}}$

$$
\widetilde{\varphi}\left(p, p_{n+1}\right)=\varphi(p)+p_{n+1} x_{(0)}^{n+1},
$$

where $x_{(0)}^{n+1}$ is a non-zero constant, and $\varphi$ a function. Then, applying Theorem 2.4 one has the $(n+1)$ dimensional Legendre submanifold $\widetilde{\mathcal{A}}_{\widetilde{\varphi}}$ generated by $-\widetilde{\varphi}$, such that

$\widetilde{\mathcal{A}}_{\widetilde{\varphi}}^{\widetilde{\mathcal{C}}}:=\Phi_{\widetilde{\mathcal{C}} \widetilde{\mathcal{A}}} \widetilde{\mathcal{A}}_{\widetilde{\varphi}}=\left\{\left(x, x^{n+1}, p, p_{n+1}, z\right) \in \widetilde{\mathcal{C}} \mid x^{i}=\frac{\partial \varphi}{\partial p_{i}}, x^{n+1}=x_{(0)}^{n+1}, z=p_{i} \frac{\partial \varphi}{\partial p_{i}}-\varphi, i \in\{1, \ldots, n\}\right\}$,

The following functions

$\widetilde{\Delta}^{0}\left(x, x^{n+1}, p, p_{n+1}, z\right):=x^{i} p_{i}+\left(x^{n+1}-x_{(0)}^{n+1}\right) p_{n+1}-\varphi(p)-z, \quad \widetilde{\Delta}^{a}\left(x, x^{n+1}, p\right):=x^{a}-\frac{x^{n+1}}{x_{(0)}^{n+1}} \frac{\partial \varphi}{\partial p_{a}}(p)$,

will be used. It follows that

$$
\left.\widetilde{\Delta}^{0}\right|_{\widetilde{\mathcal{A}}_{\tilde{\mathscr{\varphi}}}^{\tilde{C}}}=0, \quad \text { and }\left.\quad \widetilde{\Delta}^{a}\right|_{\widetilde{\mathcal{A}}_{\tilde{\mathscr{\varphi}}}^{\tilde{C}}}=0 .
$$

In addition, since the matrix components which are differentiation of $\widetilde{\varphi}$ with respect to $p$ and $p_{n+1}$ are obtained as

$$
\widetilde{g}^{\mu \nu}=\left(\begin{array}{cc}
\frac{\partial^{2} \varphi}{\partial p_{a} \partial p_{b}} & 0 \\
0 & 0
\end{array}\right), \quad a, b \in\{1, \ldots, n\}, \quad \mu, \nu \in\{1, \ldots, n+1\},
$$

the matrix $\left(\widetilde{g}_{\mu \nu}\right)$ provides neither a Riemannian metric tensor field nor a pseudo-Riemannian metric tensor field on $\widetilde{\mathcal{A}}_{\widetilde{\varphi}}$.

\subsection{Vector fields on dually flat space and their lifts in contact geometric language}

Consider an $n$-dimensional dually flat space $\left(\mathcal{M}, \nabla \mathrm{d} \psi, \nabla, \nabla^{*}\right)$, where $\psi$ is a strictly convex function giving a metric tensor field $g=\nabla \mathrm{d} \psi$. Given $\nabla$-affine coordinates $x=\left\{x^{1}, \ldots, x^{n}\right\}, \nabla$-affine coordinates $p=\left\{p_{1}, \ldots, p_{n}\right\}$ are obtained by $p_{a}=\partial \psi / \partial x^{a},(a \in\{1, \ldots, n\})$. As shown below, under the condition 
that the value of the function generating the Legendre submanifold is conserved, one way to write a dynamical system on $\mathcal{M}$

$$
\dot{x}^{a}=F_{\psi}^{a}(x), \quad \text { and } \quad \dot{p}_{a}=\frac{\mathrm{d}}{\mathrm{d} t}\left(\frac{\partial \psi}{\partial x^{a}}\right),
$$

with some $\left\{F_{\psi}^{1}, \ldots, F_{\psi}^{n}\right\}$ in a contact geometric language is to introduce a $(2 n+3)$-dimensional contact manifold $\widetilde{\mathcal{C}}$ and another convex function $\widetilde{\psi}$ that generates a new Legendre submanifold. One then needs to find an appropriate contact Hamiltonian such that some components of the contact Hamiltonian vector field being restricted to the new Legendre submanifold are identical to (46). The lift of the dynamical system to $\widetilde{\mathcal{C}}$ is then immediately obtained as the unrestricted contact Hamiltonian vector field.

The following and its counterpart are fundamental in this subsection.

Proposition 5.2. ( Vector field on $\widetilde{\mathcal{C}}$ lifted from the Legendre submanifold generated by $\psi$ ) : Let $\left\{F_{\psi}^{1}, \ldots, F_{\psi}^{n}\right\}$ be a set of functions of $x=\left\{x^{1}, \ldots, x^{n}\right\}$ on $\mathcal{A}_{\psi}$, where these functions do not identically vanish. The flow of the vector field associated with the dynamical system defined on $\mathcal{A}_{\psi}$ as

$$
X_{\psi}^{0}=\dot{x}^{a} \frac{\partial}{\partial x^{a}}, \quad \text { where } \quad \frac{\mathrm{d}}{\mathrm{d} t} x^{a}=F_{\psi}^{a}(x), \quad a \in\{1, \ldots, n\}
$$

is lifted to $\widetilde{\mathcal{C}}$ as

$$
\widetilde{X}_{\widetilde{h}, \psi}=\dot{x}^{a} \frac{\partial}{\partial x^{a}}+\dot{x}^{n+1} \frac{\partial}{\partial x^{n+1}}+\dot{p}_{a} \frac{\partial}{\partial p_{a}}+\dot{p}_{n+1} \frac{\partial}{\partial p_{n+1}}+\dot{z} \frac{\partial}{\partial z}
$$

where

$$
\begin{aligned}
& \frac{\mathrm{d} x^{a}}{\mathrm{~d} t}=F_{\psi}^{a}(x), \quad \frac{\mathrm{d} x^{n+1}}{\mathrm{~d} t}=-\frac{1}{p_{n+1}^{(0)}} \frac{\partial \psi}{\partial x^{j}} F_{\psi}^{j}, \frac{\mathrm{d} p_{n+1}}{\mathrm{~d} t}=\frac{\mathrm{d} \widetilde{\Gamma}_{\psi}}{\mathrm{d} \widetilde{\Delta}_{0}}\left(p_{n+1}^{(0)}-p_{n+1}\right), \quad \frac{\mathrm{d} z}{\mathrm{~d} t}=\widetilde{\Gamma}_{\psi}\left(\widetilde{\Delta}_{0}\right), \\
& \frac{\mathrm{d} p_{a}}{\mathrm{~d} t}=\frac{p_{n+1}}{p_{n+1}^{(0)}}\left(\frac{\partial^{2} \psi}{\partial x^{a} \partial x^{j}} F_{\psi}^{j}+\frac{\partial \psi}{\partial x^{j}} \frac{\partial F_{\psi}^{j}}{\partial x^{a}}\right)-p_{j} \frac{\partial F_{\psi}^{j}}{\partial x^{a}}+\frac{\mathrm{d} \widetilde{\Gamma}_{\psi}}{\mathrm{d} \widetilde{\Delta}_{0}}\left(\frac{\partial \psi}{\partial x^{a}}-p_{a}\right), \quad a, j \in\{1, \ldots, n\},
\end{aligned}
$$

with $\widetilde{\Gamma}_{\psi}$ being a function of $\widetilde{\Delta}_{0}$. Equivalently, these can be written as

$$
\begin{aligned}
& \frac{\mathrm{d} x^{a}}{\mathrm{~d} t}=F_{\psi}^{a}(x), \quad \frac{\mathrm{d} x^{n+1}}{\mathrm{~d} t}=\frac{-1}{p_{n+1}^{(0)}} \frac{\partial \psi}{\partial x^{j}} F_{\psi}^{j}, \quad \frac{\mathrm{d} p_{n+1}}{\mathrm{~d} t}=\frac{\mathrm{d} \widetilde{\Gamma}_{\psi}}{\mathrm{d} \widetilde{\Delta}_{0}}\left(p_{n+1}^{(0)}-p_{n+1}\right), \\
& \frac{\mathrm{d} \widetilde{\Delta}_{a}}{\mathrm{~d} t}=-\frac{\partial F_{\psi}^{j}}{\partial x^{a}} \widetilde{\Delta}_{j}-\frac{\mathrm{d} \widetilde{\Gamma}_{\psi}}{\mathrm{d} \widetilde{\Delta}_{0}} \widetilde{\Delta}_{a}, \quad \frac{\mathrm{d} \widetilde{\Delta}_{0}}{\mathrm{~d} t}=-\widetilde{\Gamma}_{\psi}\left(\widetilde{\Delta}_{0}\right),
\end{aligned}
$$

This lifted flow $\widetilde{X}_{\widetilde{h}, \psi}$, (49) and (50), is the contact Hamiltonian vector field associated to the contact Hamiltonian

$$
\widetilde{h}_{\psi}\left(x, x^{n+1}, p, p_{n+1}, z\right)=\widetilde{\Delta}_{a}\left(x, p, p_{n+1}\right) F_{\psi}^{a}(x)+\widetilde{\Gamma}_{\psi}\left(\widetilde{\Delta}_{0}\left(x, x^{n+1}, z\right)\right),
$$

and has the property

$$
\mathcal{L}_{\widetilde{X}_{\tilde{h}, \psi}} \widetilde{\psi}=0 .
$$

Proof. Substituting (51) into the high-dimensional analogue of (13), one has the component expression of the contact Hamiltonian vector field as (47) and (48). Furthermore, differentiating $\widetilde{\Delta}_{0}$ and

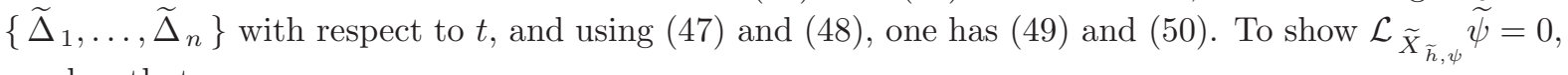
one has that

$$
\mathcal{L}_{\widetilde{X}_{\tilde{h}, \psi}} \widetilde{\psi}=\mathcal{L}_{\widetilde{X}_{\tilde{h}, \psi}}\left(\psi(x)+p_{n+1}^{(0)} x^{n+1}\right)=\dot{x}^{j} \frac{\partial \psi}{\partial x^{j}}+p_{n+1}^{(0)} \dot{x}^{n+1}=F_{\psi}^{j} \frac{\partial \psi}{\partial x^{j}}+p_{n+1}^{(0)} \dot{x}^{n+1}=0,
$$

where (49) has been used.

Remark 5.1. The functions $\left\{F_{\psi}^{1}, \ldots, F_{\psi}^{n}\right\}$ need not depend on $\psi$. 
Remark 5.2. If $F_{\psi}^{a}(x)=\left(J^{a j}(x)-R^{a j}(x)\right) \partial \psi / \partial x^{j}$ where $\left(J^{a b}\right)$ is a skew-symmetric matrix $\left(J^{a b}=\right.$ $\left.-J^{b a}\right),\left(R^{a b}\right)$ is a symmetric definite matrix, and $p_{n+1}^{(0)}>0$, then

$$
\frac{\mathrm{d}}{\mathrm{d} t} x^{n+1}>0
$$

This inequality corresponds to the statement "the entropy is increasing" found in Ref. [23].

There is a counterpart of this Proposition.

Proposition 5.3. (Vector field on $\widetilde{\mathcal{C}}$ lifted from the Legendre submanifold generated by $-\varphi$ ) : Let $\left\{F_{1}^{\varphi}, \ldots, F_{n}^{\varphi}\right\}$ be a set of functions of $p=\left\{p_{1}, \ldots, p_{n}\right\}$ on $\mathcal{A}_{\varphi}$, where these functions do not identically vanish. The flow of the vector field associated with the dynamical system defined on $\mathcal{A}_{\varphi}$ as

$$
X_{\varphi}^{0}=\dot{p}_{a} \frac{\partial}{\partial p_{a}}, \quad \text { where } \quad \frac{\mathrm{d}}{\mathrm{d} t} p_{a}=F_{a}^{\varphi}(p), \quad a \in\{1, \ldots, n\}
$$

is lifted to $\widetilde{\mathcal{C}}$ as

$$
\widetilde{X}_{\widetilde{h}, \varphi}=\dot{x}^{a} \frac{\partial}{\partial x^{a}}+\dot{x}^{n+1} \frac{\partial}{\partial x^{n+1}}+\dot{p}_{a} \frac{\partial}{\partial p_{a}}+\dot{p}_{n+1} \frac{\partial}{\partial p_{n+1}}+\dot{z} \frac{\partial}{\partial z}
$$

where

$$
\begin{gathered}
\frac{\mathrm{d} x^{a}}{\mathrm{~d} t}=\frac{x^{n+1}}{x_{(0)}^{n+1}} \frac{\partial^{2} \varphi}{\partial p_{a} \partial p_{i}} F_{i}^{\varphi}-\widetilde{\Delta}^{i} \frac{\partial F_{i}^{\varphi}}{\partial p_{a}}+\frac{\mathrm{d} \widetilde{\Gamma}^{\varphi}}{\mathrm{d} \widetilde{\Delta}^{0}}\left(\frac{\partial \varphi}{\partial p_{a}}-x^{a}\right), \quad a, i \in\{1, \ldots, n\}, \\
\frac{\mathrm{d} x^{n+1}}{\mathrm{~d} t}=\frac{\mathrm{d} \widetilde{\Gamma}^{\varphi}}{\mathrm{d} \widetilde{\Delta}^{0}}\left(x_{(0)}^{n+1}-x^{n+1}\right), \frac{\mathrm{d} p_{a}}{\mathrm{~d} t}=F_{a}^{\varphi}, \frac{\mathrm{d} p_{n+1}}{\mathrm{~d} t}=\frac{-1}{x_{(0)}^{n+1}} \frac{\partial \varphi}{\partial p_{i}} F_{i}^{\varphi}, \\
\frac{\mathrm{d} z}{\mathrm{~d} t}=\widetilde{\Delta}^{i} F_{i}^{\varphi}+\widetilde{\Gamma}^{\varphi}+\frac{x^{n+1}}{x_{(0)}^{n+1}} p_{i} \frac{\partial^{2} \varphi}{\partial p_{i} \partial p_{a}} F_{a}^{\varphi}-p_{i} \widetilde{\Delta}^{a} \frac{\partial F_{a}^{\varphi}}{\partial p_{i}}-\left[x^{i} p_{i}+\left(x^{n+1}-x_{(0)}^{n+1}\right) p_{n+1}-p_{i} \frac{\partial \varphi}{\partial p_{i}}\right] \frac{\mathrm{d} \widetilde{\Gamma}^{\varphi}}{\mathrm{d} \widetilde{\Delta}^{0}},
\end{gathered}
$$

with $\widetilde{\Gamma}^{\varphi}$ being a function of $\widetilde{\Delta}^{0}$. Equivalently, these can be written as

$$
\begin{aligned}
& \frac{\mathrm{d} p_{a}}{\mathrm{~d} t}=F_{a}^{\varphi}(p), \quad \frac{\mathrm{d} p_{n+1}}{\mathrm{~d} t}=\frac{-1}{x_{(0)}^{n+1}} \frac{\partial \varphi}{\partial p_{i}} F_{i}^{\varphi}, \quad \frac{\mathrm{d} x^{n+1}}{\mathrm{~d} t}=\frac{\mathrm{d} \widetilde{\Gamma}_{\psi}}{\mathrm{d} \widetilde{\Delta}^{0}}\left(x_{(0)}^{n+1}-x^{n+1}\right), \\
& \frac{\mathrm{d} \widetilde{\Delta}^{a}}{\mathrm{~d} t}=-\frac{\partial F_{i}^{\varphi}}{\partial p_{a}} \widetilde{\Delta}^{i}-\frac{\mathrm{d} \widetilde{\Gamma}^{\varphi}}{\mathrm{d} \widetilde{\Delta}^{0}} \widetilde{\Delta}^{a}, \quad \frac{\mathrm{d} \widetilde{\Delta}^{0}}{\mathrm{~d} t}=-\widetilde{\Gamma}_{\psi}\left(\widetilde{\Delta}^{0}\right),
\end{aligned}
$$

This lifted flow $\widetilde{X}_{\widetilde{h}, \varphi}$, (52) and (53), is the contact Hamiltonian vector field associated to the contact Hamiltonian

$$
\widetilde{h}_{\varphi}\left(x, x^{n+1}, p, p_{n+1}, z\right)=\widetilde{\Delta}^{a}\left(x, x^{n+1}, p\right) F_{a}^{\varphi}(p)+\widetilde{\Gamma}^{\varphi}\left(\widetilde{\Delta}^{0}\left(x, x^{n+1}, p, p_{n+1}, z\right)\right),
$$

and has the property

$$
\mathcal{L}_{\tilde{X}_{\tilde{h}, \varphi}} \widetilde{\varphi}=0 .
$$

Proof. A way to prove this proposition is analogous to that of Preposition 5.2 .

Remark 5.3. The functions $\left\{F_{1}^{\varphi}, \ldots, F_{n}^{\varphi}\right\}$ need not depend on $\varphi$.

Remark 5.4. If $F_{a}^{\varphi}(p)=\left(J_{a i}(p)-R_{a i}(p)\right) \partial \varphi / \partial p_{i}$ where $\left(J_{a b}\right)$ is a skew-symmetric matrix $\left(J_{a b}=-J_{b a}\right.$ ), $\left(R^{a b}\right)$ is a symmetric definite matrix, and $x_{(0)}^{n+1}>0$, then

$$
\frac{\mathrm{d}}{\mathrm{d} t} p_{n+1}>0
$$

Proposition 5.4. (Differentiation of $\widetilde{h}_{\psi}$ with respect to $t$ ) : Consider the system stated in Proposition 5.2. Then, it follows that

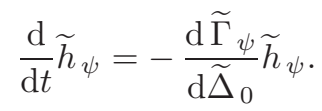


Proof. It follows from (50) that

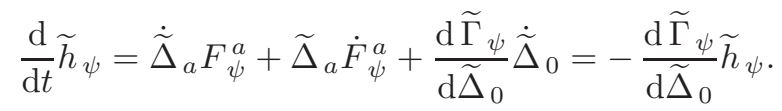

Remark 5.5. For systems where $\widetilde{\Gamma}_{\psi}\left(\widetilde{\Delta}_{0}\right)=\widetilde{\gamma}_{0} \widetilde{\Delta}_{0}$ with $\widetilde{\gamma}_{0}$ being a non-zero constant, the subsets $\left\{\left(x, x^{n+1}, p, p_{n+1}, z\right) \mid \widetilde{h}_{\psi}>0\right\} \subset \widetilde{\mathcal{C}}$ and $\left\{\left(x, x^{n+1}, p, p_{n+1}, z\right) \mid \widetilde{h}_{\psi}<0\right\} \subset \widetilde{\mathcal{C}}$ are invariant ones.

There is a counterpart of this Proposition.

Proposition 5.5. (Differentiation of $\widetilde{h}_{\varphi}$ with respect to $t$ ) : Consider the system stated in Proposition 5.3. Then, it follows that

$$
\frac{\mathrm{d}}{\mathrm{d} t} \widetilde{h}_{\varphi}=-\frac{\mathrm{d} \widetilde{\Gamma}^{\varphi}}{\mathrm{d} \widetilde{\Delta}^{0}} \widetilde{h}_{\varphi}
$$

Proof. A way to prove this is analogous to that of Proposition 5.4

Remark 5.6. For systems where $\widetilde{\Gamma}^{\varphi}\left(\widetilde{\Delta}^{0}\right)=\widetilde{\gamma}^{0} \widetilde{\Delta}^{0}$ with $\widetilde{\gamma}^{0}$ being a non-zero constant, the subsets $\left\{\left(x, x^{n+1}, p, p_{n+1}, z\right) \mid \widetilde{h}_{\varphi}>0\right\} \subset \widetilde{\mathcal{C}}$ and $\left\{\left(x, x^{n+1}, p, p_{n+1}, z\right) \mid \widetilde{h}_{\varphi}<0\right\} \subset \widetilde{\mathcal{C}}$ are invariant ones.

As shown below, the phase compressibility for a class of the lifted vector fields with $\widetilde{\Omega}_{\widetilde{\lambda}}$ does not depend on $\left\{F_{\psi}^{1}, \ldots, F_{\psi}^{n}\right\}$.

Lemma 5.1. ( Phase compressibility for $\widetilde{X}_{\widetilde{h}, \psi}$ with $\widetilde{\Omega}_{\widetilde{\lambda}}$ ): Consider the system stated in Proposition5.2. Choose $\widetilde{\Gamma}_{\psi}\left(\widetilde{\Delta}_{0}\right)=\widetilde{\gamma}_{0} \widetilde{\Delta}_{0}$ with $\widetilde{\gamma}_{0}$ being a constant. Then, the phase compressibility is calculated as

$$
\kappa_{\widetilde{\Omega}_{\tilde{\lambda}}}\left(\widetilde{X}_{\widetilde{h}, \psi}\right)=-(n+2) \widetilde{\gamma}_{0}
$$

Proof. With $\mathcal{L}_{\widetilde{X}_{\tilde{h}, \psi}} \widetilde{\lambda}=\left(\widetilde{\mathcal{R}} \widetilde{h}_{\psi}\right) \widetilde{\lambda}=-\widetilde{\gamma}_{0} \widetilde{\lambda}$ and $\mathcal{L}_{\widetilde{X}_{\tilde{h}, \psi}} \mathrm{d} \widetilde{\lambda}=\mathrm{d} \mathcal{L}_{\widetilde{X}_{\tilde{h}, \psi}} \widetilde{\lambda}=-\widetilde{\gamma}_{0} \mathrm{~d} \widetilde{\lambda}$, one has that

$$
\mathcal{L}_{\widetilde{X}_{\widetilde{h}, \psi}} \widetilde{\Omega}_{\widetilde{\lambda}}=-(n+2) \widetilde{\gamma}_{0} \widetilde{\Omega}_{\widetilde{\lambda}}
$$

Comparing (55) with (11), one completes the proof.

There is a counterpart of this Lemma.

Lemma 5.2. ( Phase compressibility for $\widetilde{X}_{\widetilde{h}, \varphi}$ with $\widetilde{\Omega}_{\widetilde{\lambda}}$ ): Consider the system stated in Proposition 5.3 . Choose $\widetilde{\Gamma}_{\varphi}\left(\widetilde{\Delta}^{0}\right)=\widetilde{\gamma}^{0} \widetilde{\Delta}^{0}$ with $\widetilde{\gamma}^{0}$ being a constant. Then, the phase compressibility is calculated as

$$
\kappa_{\widetilde{\Omega}}\left(\widetilde{X}_{\widetilde{h}, \varphi}\right)=-(n+2) \widetilde{\gamma}^{0}
$$

Proof. A way to prove this is analogous to that of Lemma 5.1

The following and its counterpart are keys to deal with statistical behaviour of the lifted vector fields.

Theorem 5.1. ( Invariant measure for $\widetilde{X}_{\widetilde{h}, \psi}$ with $\widetilde{\Omega}_{\widetilde{\lambda}}$ ) : Consider the system stated in Proposition 5.2 with $\widetilde{h}_{\psi}>0$. Choose $\widetilde{\Gamma}_{\psi}\left(\widetilde{\Delta}_{0}\right)=\widetilde{\gamma}_{0} \widetilde{\Delta}_{0}$ with $\widetilde{\gamma}_{0}$ being a non-zero constant. An invariant measure in the sense of Definition 2.3 is obtained as

$$
\widetilde{\Omega}_{\widetilde{h}, \psi}^{\operatorname{Inv}}=\widetilde{f}_{\widetilde{h}, \psi}^{\operatorname{Inv}} \widetilde{\Omega}_{\widetilde{\lambda}}, \quad \text { where } \quad \widetilde{f}_{\widetilde{h}, \psi}^{\operatorname{Inv}}=\frac{1}{\widetilde{\mathcal{Z}}_{\widetilde{h}, \psi}} \widetilde{h}_{\psi}^{-(n+2)},
$$

for the class of the form $\widetilde{f}_{\widetilde{h}, \psi}^{\operatorname{Inv}}=\widetilde{f}_{\widetilde{h}, \psi}^{\operatorname{Inv}}\left(\widetilde{h}_{\psi}\right)$ with $\widetilde{\mathcal{Z}}_{\widetilde{h}, \psi}$ being a non-zero constant.

Proof. A way to prove this is analogous to that of Theorem4.1.

Remark 5.7. Due to Remark 5.5 and the subset $\left\{\left(x, x^{n+1}, p, p_{n+1}, z\right) \mid \widetilde{h}_{\psi}>0\right\} \subset \widetilde{\mathcal{C}}$ is invariant one, one has that $\tilde{f}_{\widetilde{h}, \psi}^{\operatorname{Inv}}>0$. 
There is a counterpart of this Theorem.

Theorem 5.2. ( Invariant measure for $\widetilde{X}_{\widetilde{h}, \varphi}$ with $\widetilde{\Omega}_{\widetilde{\lambda}}$ ) : Consider the system stated in Proposition 5.3 with $\widetilde{h}_{\varphi}>0$. Choose $\widetilde{\Gamma}^{\varphi}\left(\widetilde{\Delta}^{0}\right)=\widetilde{\gamma}^{0} \widetilde{\Delta}^{0}$ with $\widetilde{\gamma}^{0}$ being a non-zero constant. An invariant measure in the sense of Definition 2.3 is obtained as

$$
\widetilde{\Omega}_{\widetilde{h}, \varphi}^{\operatorname{Inv}}=\widetilde{f}_{\widetilde{h}, \varphi}^{\operatorname{Inv}} \widetilde{\Omega}_{\widetilde{\lambda}}, \quad \text { where } \quad \widetilde{f}_{\widetilde{h}, \varphi}^{\operatorname{Inv}}=\frac{1}{\widetilde{\mathcal{Z}}_{\widetilde{h}, \varphi}} \widetilde{h}_{\varphi}^{-(n+2)},
$$

for the class of the form $\widetilde{f}_{\widetilde{h}, \varphi}^{\operatorname{Inv}}=\widetilde{f}_{\widetilde{h}, \varphi}^{\operatorname{Inv}}\left(\widetilde{h}_{\varphi}\right)$ with $\widetilde{\mathcal{Z}}_{\widetilde{h}, \varphi}$ being a non-zero constant.

Proof. A way to prove this is analogous to that of Theorem 4.1 .

Remark 5.8. Due to Remark 5.6 and the subset $\left\{\left(x, x^{n+1}, p, p_{n+1}, z\right) \mid \widetilde{h}_{\varphi}>0\right\} \subset \widetilde{\mathcal{C}}$ is invariant one, one has that $\tilde{f}_{\widetilde{h}, \varphi}^{\operatorname{Inv}}>0$.

The vector field on the Legendre submanifold $\widetilde{\mathcal{A}} \widetilde{\mathcal{\psi}}$ is immediately obtained from the restriction of $\widetilde{X}_{\widetilde{h}, \psi}$ as follows.

Proposition 5.6. ( Restriction of $\widetilde{X}_{\widetilde{h}, \psi}$ on $\widetilde{\mathcal{A}}_{\widetilde{\psi}}^{\widetilde{\mathcal{C}}}$ ) : Let $\widetilde{X}_{\widetilde{h}, \psi}$ be the contact Hamiltonian vector field on $\widetilde{\mathcal{C}}$ associated with $\widetilde{h}_{\psi}$ in (51). If $\widetilde{\Gamma}_{\psi}$ is such that $\widetilde{\Gamma}_{\psi}(0)=0$ and $\widetilde{\Gamma}_{\psi}\left(\widetilde{\Delta}^{0}\right) \neq 0$ for $\widetilde{\Delta}^{0} \neq 0$, then the components of the vector field $\left.\widetilde{X}_{\widetilde{h}, \psi}\right|_{\widetilde{\mathcal{A}} \widetilde{\tilde{\mathcal{C}}}}$ are written as

$$
\frac{\mathrm{d} x^{a}}{\mathrm{~d} t}=F_{\psi}^{a}(x), \frac{\mathrm{d} x^{n+1}}{\mathrm{~d} t}=\frac{-1}{p_{n+1}^{(0)}} \frac{\partial \psi}{\partial x^{j}} F_{\psi}^{j}, \frac{\mathrm{d} p_{n+1}}{\mathrm{~d} t}=0, \frac{\mathrm{d} z}{\mathrm{~d} t}=0, \frac{\mathrm{d} p_{a}}{\mathrm{~d} t}=\frac{\partial^{2} \psi}{\partial x^{a} \partial x^{j}} F_{\psi}^{j}, a, j \in\{1, \ldots, n\} .
$$

Equivalently, these can be written as

$$
\frac{\mathrm{d} x^{a}}{\mathrm{~d} t}=F_{\psi}^{a}(x), \quad \frac{\mathrm{d} x^{n+1}}{\mathrm{~d} t}=\frac{-1}{p_{n+1}^{(0)}} \frac{\partial \psi}{\partial x^{j}} F_{\psi}^{j}, \quad \frac{\mathrm{d} p_{n+1}}{\mathrm{~d} t}=0, \frac{\mathrm{d} \widetilde{\Delta}_{0}}{\mathrm{~d} t}=0, \quad \frac{\mathrm{d} \Delta_{a}}{\mathrm{~d} t}=0, \quad a \in\{1, \ldots, n\}
$$

where $\Delta_{a}$ has been defined in (18) as

$$
\Delta_{a}=\frac{\partial \psi}{\partial x^{a}}-p_{a}
$$

Furthermore, this restricted contact Hamiltonian vector field has the property

$$
\mathcal{L}_{\left.\widetilde{X}_{\tilde{h}, \psi}\right|_{\tilde{\mathcal{A}} \tilde{\tilde{\psi}}} ^{\tilde{\mathcal{C}}}} \widetilde{\psi}=0
$$

Proof. Components of the restricted vector field are easily obtained from Proposition 5.2 The property (56) is proved by straightforward calculations as

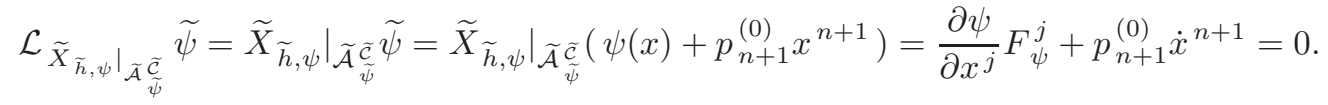

There is a counterpart of this proposition. The vector field on the Legendre submanifold $\mathcal{A}_{\widetilde{\varphi}}^{\widetilde{\mathcal{C}}}$ is immediately obtained from the restriction of $\widetilde{X}_{\widetilde{h}, \varphi}$ as follows.

Proposition 5.7. ( Restriction of $\widetilde{X}_{\widetilde{h}, \varphi}$ on $\left.\widetilde{\mathcal{A}}_{\widetilde{\varphi}}^{\widetilde{\mathcal{C}}}\right)$ : Let $\widetilde{X}_{\widetilde{h}, \varphi}$ be the contact Hamiltonian vector field on $\widetilde{\mathcal{C}}$ associated with $\widetilde{h}_{\varphi}$ in 54). If $\widetilde{\Gamma}^{\varphi}$ is such that $\widetilde{\Gamma}^{\varphi}(0)=0$ and $\widetilde{\Gamma}^{\varphi}\left(\widetilde{\Delta}^{0}\right) \neq 0$ for $\widetilde{\Delta}^{0} \neq 0$, then the components of the vector field $\left.\widetilde{X}_{\widetilde{h}, \varphi}\right|_{\widetilde{\mathcal{A}}_{\tilde{\varphi}}^{\mathcal{C}}}$ are written as

$$
\frac{\mathrm{d} p_{a}}{\mathrm{~d} t}=F_{a}^{\varphi}(p), \frac{\mathrm{d} p_{n+1}}{\mathrm{~d} t}=\frac{-1}{x_{(0)}^{n+1}} \frac{\partial \varphi}{\partial p_{i}} F_{i}^{\varphi}, \frac{\mathrm{d} x^{n+1}}{\mathrm{~d} t}=0, \frac{\mathrm{d} z}{\mathrm{~d} t}=0, \frac{\mathrm{d} x^{a}}{\mathrm{~d} t}=\frac{\partial^{2} \varphi}{\partial p_{a} \partial p_{i}} F_{i}^{\varphi}, a, i \in\{1, \ldots, n\} .
$$


Equivalently, these can be written as

$$
\frac{\mathrm{d} p_{a}}{\mathrm{~d} t}=F_{a}^{\varphi}(p), \quad \frac{\mathrm{d} p_{n+1}}{\mathrm{~d} t}=\frac{-1}{x_{(0)}^{n+1}} \frac{\partial \varphi}{\partial p_{a}} F_{a}^{\varphi}, \quad \frac{\mathrm{d} p_{n+1}}{\mathrm{~d} t}=0, \frac{\mathrm{d} \widetilde{\Delta}^{0}}{\mathrm{~d} t}=0, \quad \frac{\mathrm{d} \Delta^{a}}{\mathrm{~d} t}=0, \quad a \in\{1, \ldots, n\}
$$

where $\Delta^{a}$ has been defined in (19) as

$$
\Delta^{a}=x^{a}-\frac{\partial \varphi}{\partial p_{a}}
$$

Furthermore, this restricted contact Hamiltonian vector field has the property

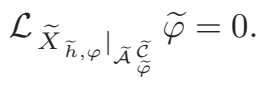

Proof. A way to prove this is analogous to that of Proposition 5.6.

\subsection{Applications to electric circuit models in a thermal environment}

In this subsection, Proposition 5.6 is applied to systems consisting of the RC, RL and RLC circuit models ( see Definitions 3.1, 3.2, 3.3) and an environment with the constant temperature $T_{0}$.

First, an extension of the RC circuit model is considered.

Definition 5.2. ( $R C$ circuit model in a thermal environment) : Consider the $R C$ circuit model stated in Definition 3.1. In addition, let $T_{0}>$ be a constant temperature, $S$ entropy, and $\mathcal{H}_{\mathrm{C}, T_{0}}^{\mathrm{tot}}$ the total energy

$$
\mathcal{H}_{\mathrm{C}, T_{0}}^{\text {tot }}\left(Q_{\mathrm{C}}, S\right)=\mathcal{H}_{\mathrm{C}}\left(Q_{\mathrm{C}}\right)+T_{0} S
$$

where $\mathcal{H}_{\mathrm{C}}\left(Q_{\mathrm{C}}\right)$ is given in (29). Then, the space $\mathbb{R}^{5}$ whose coordinates are $\left(Q_{\mathrm{C}}, S, V_{\mathrm{C}}, T, z_{\mathrm{C}}\right)$ is referred to as the extended thermodynamic phase space, where $T$ is a 1-dimensional coordinate for expressing temperature, and $z_{\mathrm{C}}$ is a 1-dimensional coordinate for expressing energy. In addition, the contact Hamiltonian vector field restricted to the Legendre submanifold generated by $\mathcal{H}_{\mathrm{C}, T_{0}}^{\text {tot }},\left.\widetilde{X}_{\widetilde{h}, \psi}\right|_{\widetilde{\mathcal{A}}_{\widetilde{\psi}}^{\widetilde{\mathcal{c}}}}$ in Proposition 5.6. is referred to as the RC circuit model in the thermal environment with temperature $T_{0}$. Here $\psi$ and $\widetilde{\psi}$ are identified with $\mathcal{H}_{\mathrm{C}}$ and $\mathcal{H}_{\mathrm{C}, T_{0}}^{\mathrm{tot}}$, respectively, and $F_{\psi}^{1}$ is identified with $F_{\mathrm{C}}$ in Proposition 3.3 .

Remark 5.9. If $T_{0}=0$, then the $\mathrm{RC}$ circuit model in the thermal environment with temperature $T_{0}$ reduces to the system in Definition 3.1 .

Remark 5.10. This system proposed in this Definition is similar to that of Ref. [23].

Remark 5.11. On the Legendre submanifold generated by $\mathcal{H}_{\mathrm{C}, T_{0}}^{\text {tot }}$, the relations

$$
V_{\mathrm{C}}=\frac{\mathrm{d} \mathcal{H}_{\mathrm{C}}}{\mathrm{d} Q_{\mathrm{C}}}=\frac{Q_{\mathrm{C}}}{C}, \quad T=T_{0}, \quad \text { and } \quad z_{\mathrm{C}}=\mathcal{H}_{\mathrm{C}, T_{0}}^{\mathrm{tot}},
$$

hold in the extended thermodynamic phase space, due to (45).

Then, one has the following proposition.

Proposition 5.8. ( Total energy and entropy production in the $R C$ circuit model in a thermal environment ): For the $R C$ circuit model in the thermal environment with $T_{0}$, one has the equations for $Q_{\mathrm{C}}, V_{\mathrm{C}}$ and $S$ as

$$
\frac{\mathrm{d} Q_{\mathrm{C}}}{\mathrm{d} t}=-\frac{Q_{\mathrm{C}}}{R C}, \quad \frac{\mathrm{d} V_{\mathrm{C}}}{\mathrm{d} t}=-\frac{Q_{\mathrm{C}}}{R C^{2}}, \quad \frac{\mathrm{d} S}{\mathrm{~d} t}=\frac{1}{T_{0}} \frac{Q_{\mathrm{C}}^{2}}{R C^{2}}>0, \quad V_{\mathrm{C}}=Q_{\mathrm{C}} V_{\mathrm{C}} .
$$

In addition, it follows that

$$
\frac{\mathrm{d}}{\mathrm{d} t} \mathcal{H}_{\mathrm{C}, T_{0}}^{\mathrm{tot}}=0
$$

Proof. The equations for $Q_{\mathrm{C}}, V_{\mathrm{C}}$, and $S$ are obtained by substituting the identifications, $n=1$, and

$$
\begin{gathered}
x^{1}=Q_{\mathrm{C}}, \quad p_{1}=V_{\mathrm{C}}, \quad x^{2}=S, \quad p_{2}^{(0)}=T_{0}, \\
\psi\left(x^{1}\right)=\mathcal{H}_{\mathrm{C}}\left(Q_{\mathrm{C}}\right), \quad \widetilde{\psi}\left(x^{1}, x^{2}\right)=\mathcal{H}_{\mathrm{C}, T_{0}}^{\text {tot }}\left(Q_{\mathrm{C}}, S\right), \quad F_{\psi}^{1}\left(x^{1}\right)=-\frac{Q_{\mathrm{C}}}{R C},
\end{gathered}
$$

into the equations in Proposition 5.6 . 
Remark 5.12. A physical interpretation of the equation involving $\mathrm{d} S / \mathrm{d} t$ is the entropy production.

Second, an extension of the RL circuit model is considered.

Definition 5.3. ( $R L$ circuit model in a thermal environment) : Consider the RL circuit model stated in Definition 3.2. In addition, let $T_{0}>$ be a constant temperature, $S$ entropy, and $\mathcal{H}_{\mathrm{L}, T_{0}}^{\mathrm{tot}}$ the total energy

$$
\mathcal{H}_{\mathrm{L}, T_{0}}^{\text {tot }}\left(N_{\mathrm{L}}, S\right)=\mathcal{H}_{\mathrm{L}}\left(N_{\mathrm{L}}\right)+T_{0} S,
$$

where $\mathcal{H}_{\mathrm{L}}\left(N_{\mathrm{L}}\right)=\sup _{I_{\mathrm{L}}}\left(I_{\mathrm{L}} N_{\mathrm{L}}-\mathcal{H}_{\mathrm{L}}^{*}\right)$, and $\mathcal{H}_{\mathrm{L}}^{*}\left(I_{\mathrm{L}}\right)$ is given in (32). Then, the space $\mathbb{R}^{5}$ whose coordinates are $\left(N_{\mathrm{L}}, S, I_{\mathrm{L}}, T, z_{\mathrm{L}}\right)$ is referred to as the extended thermodynamic phase space, where $T$ is a 1-dimensional coordinate for expressing temperature, and $z_{\mathrm{L}}$ is a 1-dimensional coordinate for expressing energy. In addition, the contact Hamiltonian vector field restricted to the Legendre submanifold generated by $\mathcal{H}_{\mathrm{L}, T_{0}}^{\mathrm{tot}},\left.\widetilde{X}_{\widetilde{h}, \psi}\right|_{\widetilde{\mathcal{A}}_{\tilde{\psi}}^{\tilde{\psi}}}$ in Proposition [5.6, is referred to as the $R L$ circuit model in the thermal environment with temperature $T_{0}$. Here $\psi$ and $\widetilde{\psi}$ are identified with $\mathcal{H}_{\mathrm{L}}$ and $\mathcal{H}_{\mathrm{L}, T_{0}}^{\mathrm{tot}}$, respectively, and $F_{\psi}^{1}\left(x^{1}\right)=-R N_{\mathrm{L}} / L$.

Remark 5.13. If $T_{0}=0$, then the RL circuit model in the thermal environment with temperature $T_{0}$ reduces to the system in Definition 3.2

Remark 5.14. This system proposed in this Definition is similar to that of Ref. 23.

Remark 5.15. On the Legendre submanifold generated by $\mathcal{H}_{\mathrm{L}, T_{0}}^{\text {tot }}$, the relations

$$
I_{\mathrm{L}}=\frac{\mathrm{d} \mathcal{H}_{\mathrm{L}}}{\mathrm{d} N_{\mathrm{L}}}=\frac{N_{\mathrm{L}}}{L}, \quad T=T_{0}, \quad \text { and } \quad z_{\mathrm{L}}=\mathcal{H}_{\mathrm{L}, T_{0}}^{\text {tot }},
$$

hold in the extended thermodynamic phase space, due to (45).

Then, one has the following proposition.

Proposition 5.9. ( Total energy and entropy production in the $R L$ circuit model in a thermal environment ) : For the $R L$ circuit model in the thermal environment with $T_{0}$, one has the equations for $N_{\mathrm{L}}, I_{\mathrm{L}}$ and $S$ as

$$
\frac{\mathrm{d} N_{\mathrm{L}}}{\mathrm{d} t}=-R I_{\mathrm{L}}, \quad \frac{\mathrm{d} I_{\mathrm{L}}}{\mathrm{d} t}=-\frac{R}{L} I_{\mathrm{L}}, \quad \frac{\mathrm{d} S}{\mathrm{~d} t}=\frac{R I_{\mathrm{L}}}{T_{0}} \frac{N_{\mathrm{L}}}{L}=\frac{R I_{\mathrm{L}}^{2}}{T_{0}}>0, \quad I_{\mathrm{L}}=\frac{N_{\mathrm{L}}}{L} .
$$

In addition, it follows that

$$
\frac{\mathrm{d}}{\mathrm{d} t} \mathcal{H}_{\mathrm{L}, T_{0}}^{\mathrm{tot}}=0
$$

Proof. The equations for $N_{\mathrm{L}}, I_{\mathrm{L}}$, and $S$ are obtained by substituting the identifications, $n=1$, and

$$
\begin{gathered}
x^{1}=N_{\mathrm{L}}, \quad p_{1}=I_{\mathrm{L}}, \quad x^{2}=S, \quad p_{2}^{(0)}=T_{0}, \\
\psi\left(x^{1}\right)=\mathcal{H}_{\mathrm{L}}\left(N_{\mathrm{L}}\right), \quad \widetilde{\psi}\left(x^{1}, x^{2}\right)=\mathcal{H}_{\mathrm{L}, T_{0}}^{\text {tot }}\left(N_{\mathrm{L}}, S\right), \quad F_{\psi}^{1}\left(x^{1}\right)=-\frac{R}{L} N_{\mathrm{L}},
\end{gathered}
$$

into the equations in Proposition 5.6

Third, an extension of the RLC circuit model is considered.

Definition 5.4. ( $R L C$ circuit model in a thermal environment) : Consider the $R L C$ circuit model stated in Definition 3.3. In addition, let $T_{0}>$ be a constant temperature, $S$ entropy, and $\mathcal{H}^{\text {tot }}$ the total energy

$$
\mathcal{H}_{T_{0}}^{\text {tot }}(Q, N, S)=\mathcal{H}(Q, N)+T_{0} S,
$$

where $\mathcal{H}(Q, N)$ is given in (35). Then, the space $\mathbb{R}^{7}$ whose coordinates are $(Q, N, S, V, I, T, z)$ is referred to as the extended thermodynamic phase space, where $T$ is a 1-dimensional coordinate for expressing temperature, and $z$ is a 1-dimensional coordinate for expressing energy. In addition, the contact Hamiltonian vector field restricted to the Legendre submanifold generated by $\mathcal{H}_{T_{0}}^{\text {tot }},\left.\widetilde{X}_{\widetilde{h}, \psi}\right|_{\widetilde{\mathcal{A}}_{\tilde{\psi}}^{\mathcal{c}}}$ in Proposition [5.6], is referred to as the $R L C$ circuit model in the thermal environment with temperature $T_{0}$. Here $\psi$ and $\widetilde{\psi}$ are identified with $\mathcal{H}$ and $\mathcal{H}_{T_{0}}^{\text {tot }}$, respectively, and $F_{\psi}^{1}(x)=N / L, F_{\psi}^{2}(x)=-Q / C-R N / L$. 
Remark 5.16. If $T_{0}=0$, then the RLC circuit model in the thermal environment with temperature $T_{0}$ reduces to the system in Definition 3.1 .

Remark 5.17. This system proposed in this Definition is similar to that of Ref. 23].

Remark 5.18. On the Legendre submanifold generated by $\mathcal{H}_{T_{0}}^{\text {tot }}$, the relations

$$
V=\frac{\partial \mathcal{H}}{\partial Q}=\frac{Q}{C}, \quad I=\frac{\partial \mathcal{H}}{\partial N}=\frac{N}{L}, \quad T=T_{0}, \quad \text { and } \quad z=\mathcal{H}_{T_{0}}^{\text {tot }}
$$

hold in the extended thermodynamic phase space, due to (45).

Then, one has the following proposition.

Proposition 5.10. ( Total energy and entropy production in the RLC circuit model in a thermal environment ) : For the $R L C$ circuit model in the thermal environment with $T_{0}$, one has the equations for $Q, N, V, I$ and $S$ as

$\frac{\mathrm{d} Q}{\mathrm{~d} t}=I, \quad \frac{\mathrm{d} N}{\mathrm{~d} t}=-V-R I, \quad \frac{\mathrm{d} V}{\mathrm{~d} t}=\frac{I}{C}, \quad \frac{\mathrm{d} I}{\mathrm{~d} t}=-\frac{V}{L}-\frac{R}{L} I, \quad \frac{\mathrm{d} S}{\mathrm{~d} t}=\frac{R}{T_{0}} I^{2}>0, \quad V=\frac{Q}{C}, \quad I=\frac{N}{L}$.

In addition, it follows that

$$
\frac{\mathrm{d}}{\mathrm{d} t} \mathcal{H}_{T_{0}}^{\text {tot }}=0
$$

Proof. The equations for $Q, N, V, I$, and $S$ are obtained by substituting the identifications, $n=2$, and

$$
\begin{gathered}
x^{1}=Q, \quad x^{2}=N, \quad x^{3}=S, \quad p_{1}=V, \quad p_{2}=I, \quad p_{3}^{(0)}=T_{0}, \\
\psi\left(x^{1}, x^{2}\right)=\mathcal{H}(Q, N), \widetilde{\psi}\left(x^{1}, x^{2}, x^{3}\right)=\mathcal{H}_{T_{0}}^{\operatorname{tot}}(Q, N, S), \quad F_{\psi}^{1}\left(x^{1}, x^{2}\right)=\frac{N}{L}, F_{\psi}^{2}\left(x^{1}, x^{2}\right)=-\frac{Q}{C}-\frac{R}{L} N,
\end{gathered}
$$

into the equations in Proposition5.6

Remark 5.19. A physical interpretation of the equation involving $\mathrm{d} S / \mathrm{d} t$ is the entropy production. This equation is the same as that of Ref. 27. When $R=0$, the entropy production vanishes.

\section{Concluding remarks}

This paper offers contact geometric descriptions of vector fields on dually flat spaces. This offer provides a view point that some ideas in information geometry can be written by means of contact geometric tools. Based on these descriptions, basic properties of vector fields on contact manifolds lifted from Legendre submanifolds have been investigated. Throughout this paper, Legendre duality has explicitly been stated. In addition, some applications of the developed general theories to science and foundation of engineering have also been given.

There are some potential future works that follow from this paper. One is to compare this work with other existing works. Such other works include the work on symplectic geometry adopted to a dually flat space discussed in Ref. 28], and relaxation dynamics on a statistical manifold in Ref. 29. In addition, it is interesting to construct the vector field associated with the Brayton-Moser equations on a dually flat space, where the Brayton-Moser equations describe a class of electric circuit models 23]. Another important future work is to import mathematical findings in contact geometry and topology to science and engineering. We believe that the elucidation of these remaining questions will develop the geometric theories in mathematical sciences and foundation of engineering.

\section{Acknowledgments}

The author would like to thank Y. Shikano ( Institute for Molecular Science ) and K. Umeno ( Kyoto University ) for supporting my work, and thank T. Wada, M. Koga, Y. Nakata, D. Tarama, and an anonymous referee for giving various comments on this work. 


\section{References}

[1] V.I. Arnold, Mathematical Methods of Classical Mechanics (Berlin: Springer ), (1976).

[2] R. Hermann, Geometry, Systems and Physics (New York: Dekker), (1973).

[3] R. Mrugala, Suken kokyuroku, 1142, 167-181, (2000).

[4] D. Eberard, B.M. Maschke, and A.J. Van Der Schaft, Rep. Math. Phys. 60, 175-198, (2007).

[5] A. Bravetti and C.S. Lopez-Monsalvo, and F. Nettel, Ann. Phys. 361, 377-400 (2015).

[6] S. Goto, J. Math. Phys. 56, 073301, (2015).

[7] R. Mrugala, J.D. Nulton, J.C. Schon and P. Salamon, Phys. Rev. A 41, 3156-3160, (1990).

[8] J. Jurkowski, Phys. Rev. E 62, 1790-1798, (2000).

[9] A. Bravetti and C.S. Lopez-Monsalvo, J. Phys. A 48, 125206, (2015) ; e-print arXiv : $1408.5443 v 3$.

[10] R. Ghrist, Handbook of Mathematical Fluid Dynamics, (Elsevier, 2007), 4, Chapter 1, 1-37, (2007).

[11] M. Dahl, Progress in Electromagnetic Research, PIER 46, 77-104, (2004).

[12] T. Ohsawa, Automatica, 55, 1-5, (2015).

[13] A. Bravetti, and D. Tapias, J. Phys. A 48, 245001, (2014) ; e-print arXiv 1412.0026v2.

[14] S.I. Amari and H. Nagaoka, Methods of Information Geometry, Trans. Math. Monogr. vol 191 (Providence:American Mathematical Society) (2000).

[15] A. Facache, V.S.D.S. Martins, D.Dochain, and B. Maschke, IEEE Trans. Automatic Control, 54, 2341-2351, (2009).

[16] H.R. Estay, B.Mashke, and D. Sbarbaro, Preprints of the 18th IFAC World Congress, Mirano (Italy) Aug. 28-Sep.2, (2011).

[17] G.S. Ezra, J. Math. Chem., 32, 339-359, (2002).

[18] G.S. Ezra, J. Math. Chem., 35, 29-53, (2004).

[19] R. Mrugala, J.D. Nulton, J.C. Schon and P. Salamon, Rep. Math. Phys. 29, 109-121, (1991).

[20] A. Ohara, N. Sunada, and S. Amari, Linear algebra Appl., 247, 31-53, (1996).

[21] A. Fujiwara and S.I. Amari, Physica D. 80 317-327, (1995).

[22] Y. Nakamura, Japan. J. Indust. Appl. Mech., 11, 21-30, (1994).

[23] D. Eberard, B.M. Maschke, and A.J. Van Der Schaft, Proceedings of the 17th International Symposium on Mathematical Theory of Networks and Systems, Kyoto, Japan, July 24-28, (2006).

[24] M.W. Hirsch and S. Smale, Differential equations, dynamical systems, and linear algebra, Academic Press, (1974).

[25] L. Onsager and S. Machlup, Phys. Rev. 91, 1505-1512, (1953).

[26] D. Eberard, B.M. Maschke, and A.J. Van Der Schaft, Proc. 44th Conference on Decision and Control, and the European Control Conference (IEEE 2005), 5977-5982.

[27] J.H. Doty and K.L. Yerkes, Proceedings of 44th AIAA (American Institute of Aeronautics \& Astronautics, 2011).

[28] T. Noda, J. Aust. Math. Soc. 90, 371-384, (2011).

[29] A. Ohara and T. Wada, J. Phys. A 43, 035002 (18pages), (2010). 